Copyright

by

Casey Megan Farmer

2015 
The Thesis Committee for Casey Farmer certifies that this is the approved version of the following thesis:

\title{
Visualization of Auditory Masking for Firefighter Alarm Detection
}

\author{
APPROVED BY \\ SUPERVISING COMMITTEE:
}

Ofodike A. Ezekoye, Supervisor

Preston S. Wilson, Co-supervisor 


\title{
Visualization of Auditory Masking for Firefighter Alarm Detection
}

\author{
by
}

Casey Megan Farmer, B.S.Arch.E.

\author{
THESIS \\ Presented to the Faculty of the Graduate School of \\ The University of Texas at Austin \\ in Partial Fulfillment \\ of the Requirements \\ for the Degree of \\ MASTER OF SCIENCE IN ENGINEERING
}

THE UNIVERSITY OF TEXAS AT AUSTIN

May 2015 


\section{Acknowledgments}

The author would like to thank the advising professors on the PASS Audibility Project, Dr. Ofodike A. Ezekoye and Dr. Preston S. Wilson, as well as the previous researchers, Joelle I. Suits and Mustafa Z. Abbasi. Additional thanks are due to the US Department of Homeland Security (DHS), the Federal Emergency Management Agency (FEMA) and their subsidiary organization, the Fire Protection Research Foundation (FPRF) for funding the PASS Audibility Project, and to Casey Grant of the National Fire Protection Association (NFPA) for his coordination between all involved parties. 


\title{
Visualization of Auditory Masking for Firefighter Alarm Detection
}

\author{
Casey Megan Farmer, M.S.E. \\ The University of Texas at Austin, 2015 \\ Supervisors: Ofodike A. Ezekoye \\ Preston S. Wilson
}

\begin{abstract}
An essential piece of firefighter equipment is the Personal Alert Safety System (PASS), which emits an alarm when a firefighter has been inactive for a specified period of time and is used to find and rescue downed firefighters. The National Institute for Occupational Safety and Health (NIOSH) firefighter fatality reports suggest that there have been instances when the PASS alarm is not audible by other firefighters on the scene. This thesis seeks to use acoustic models to measure the sound pressure level of various signals throughout a structure. With this information, a visual representation will be created to map where a PASS alarm is audible and where it is masked by noise sources. This thesis presents an initial audibility study, including temporal masking and frequency analysis. The results are compared to auralizations and experimental data. Some other potential applications will be briefly explored.
\end{abstract}




\section{Table of Contents}

Acknowledgments iv

Abstract $\quad$ v

List of Tables $\quad$ ix

List of Figures $\quad$ X

Chapter 1. Introduction 1

1.1 Literature Review . . . . . . . . . . . . . . . 2

1.1.1 Line of Duty Death Reports . . . . . . . . . . . . . 5

1.2 PASS Audibility Study . . . . . . . . . . . . . . . . 6

1.3 Overview of Work Performed . . . . . . . . . . . . . . . 8

1.3.1 Acoustical Analysis of Firefighting Equipment . . . . . . 8

1.3.2 Modeling and Validation of Sound Transmission . . . . 10

1.3.3 Physiological Acoustics and Audiology Testing . . . . . 14

1.3.4 Conclusion .................. 15

$\begin{array}{llr}\text { Chapter 2. PASS Localization Tests } & 17\end{array}$

2.1 Background .................... . . 17

2.2 Methodology . . . . . . . . . . . . . . . . 18

2.2.1 Scenarios . . . . . . . . . . . . . . . . . 19

2.2 .2 Test Layouts . . . . . . . . . . . . . . . . . . . . . . . 19

2.2.2.1 Austin Fire Department . . . . . . . . . 20

2.2.2.2 Glendale Fire Department . . . . . . . . . . 21

2.2.2.3 Oklahoma City Fire Department . . . . . . . 23

2.3 Summary of Results . . . . . . . . . . . . . . . . . 25

2.3.1 Need for Masking Visualization . . . . . . . . . . . 26 
Chapter 3. Auralization Mapping 29

3.1 Methodology . . . . . . . . . . . . . . . . . . . . . 29

3.1 .1 Ray Tracing . . . . . . . . . . . . . . . . 29

3.1 .2 Beamtracing . . . . . . . . . . . . . 31

3.1.3 Finite Element Methods . . . . . . . . . . . . . . . . 32

3.1.3.1 Fourier Synthesis . . . . . . . . . . . . . . 34

3.2 Sound Field for PASS Alarm . . . . . . . . . . . . . . . . . . . 40

3.3 Sound Fields for Noise Sources . . . . . . . . . . . . . . . . . . 42

3.4 Field where PASS is in Audible Range . . . . . . . . . . . . . 43

3.5 Potential for Auralization . . . . . . . . . . . . . . . 43

3.6 Conclusion . . . . . . . . . . . . . . . . . . 45

Chapter 4. Improved Boundary Conditions 46

4.1 Background of Gypsum Wallboard Chemistry . . . . . . . . . 46

4.2 Fire Model . . . . . . . . . . . . . . . . . . . . . 47

4.3 Acoustic Properties . . . . . . . . . . . . . . . 51

4.3.1 Impedance Tube Methods . . . . . . . . . . . . . . . 52

4.3.1.1 ETS-Lindgren Impedance Tube . . . . . . . . 53

4.3.2 Impedance Tube Procedure . . . . . . . . . . . . . . . 55

4.4 Measurements . . . . . . . . . . . . . . . . . 56

4.4.1 Impedance Tube Benchmarking Tests . . . . . . . . . 56

4.4 .2 Gypsum Measurements . . . . . . . . . . . . 58

4.4.3 Burned Gypsum Measurements . . . . . . . . . . . . . 60

4.5 Conclusion ...................... 61

$\begin{array}{lll}\text { Chapter 5. Conclusion } & 68\end{array}$

$\begin{array}{ll}\text { Appendices } & \mathbf{7 0}\end{array}$

Appendix A. MATLAB Code for COMSOL Model of AFD Of$\begin{array}{ll}\text { fice Building } & 71\end{array}$

Appendix B. MATLAB Code for Fourier Synthesis of AFD Office Building 
Appendix C. MATLAB Code for Heat Transfer Model 92

$\begin{array}{ll}\text { Bibliography } & 96\end{array}$

viii 


\section{List of Tables}

3.1 Necessary Signal Processing Values . . . . . . . . . . . . . . . 37

4.1 Resonance Frequencies of Tube . . . . . . . . . . . . . . . . 59 


\section{List of Figures}

1.1 Front perspective view of an MSA SCBA. . . . . . . . . . . . . . 4

1.2 NIOSH measured levels for specific fire vehicle. . . . . . . . . . . 9

1.3 Example of sound refraction in the presence of a sound speed gradient. 13

1.4 Example of changing transfer function during a fire. . . . . . . . . . 14

2.1 The floorplan of the AFD portable office building with the location of relevant sound sources. . . . . . . . . . . . . . . . . .

2.2 The floorplan of the GFD warehouse space with the location of relevant sound sources. . . . . . . . . . . . . . . . .

2.3 The floorplan of the OKCFD warehouse space with the location of relevant sound sources. . . . . . . . . . . . . . . .

2.4 Sample paths with and without background noise. The asterisk represents where the firefighter first alerted to audible detection of the alarm signal. . . . . . . . . . . . . .

3.1 Ecotect ray tracing example in AFD office building. . . . . . . .

3.2 COMSOL solution of square room example at $100 \mathrm{~Hz}$. The x-axis marks the width of the room and the $y$-axis marks the height of the room, each equal to 5 meters. The pressure gradient is given in decibels, scaled relative to a finite amplitude pulse in a room with lossless boundaries. . . . . . . . . . . . . . . .

3.3 Time-averaged modal response of square room example. The pressure peaks (red) and nulls (blue) represent the $2 D$ modal structure of the space. The $x$-axis marks the width of the room and the $y$-axis marks the height of the room, each equal to 5 meters. The pressure gradient is given in decibels, scaled relative to a finite amplitude pulse in a room with lossless boundaries. . . . . . . . . . . . .

3.4 Time-averaged modal response to finite amplitude impulse at PASS location, layered on top of the floorplan shown in Figure 2.1. The pressure gradient is given in decibels, scaled relative to the finite amplitude pulse in a room with lossless boundaries. . . . . . . .

4.1 Schematic of impedance tube analysis. Taken from ASTM E1050-98. 53 
4.2 The ETS-Lindgren High-Frequency Impedance Tube. . . . . . . . 54

4.3 Measured absorption coefficient of anechoic foam sample. . . . . . 62

4.4 Measured specific acoustic impedance of a rigid stop. . . . . . . . 63

4.5 Measured absorption coefficient of gypsum wallboard sample. . . 64

4.6 Measured absorption coefficient of gypsum wallboard sample with shorter backing plate. . . . . . . . . . . . . 65

4.7 Measured absorption coefficient of burned gypsum wallboard sample. . . . . . . . . . . . . . . 66

4.8 Comparison of the measured absorption coefficients of burned and unburned gypsum samples. Curves are nearly identical. . . . . . 


\section{Chapter 1}

\section{Introduction}

The purpose of the Personal Alert Safety System (PASS) warning device is quite simple: it is intended to be a reliable method of alerting nearby

fireground personnel to firefighters who have become disoriented or trapped in a structure and need assistance. A small electronic motion-sensing device is worn by a firefighter. If the device senses a lack of motion, it sounds a 95-dB, multiple-frequency alarm until motion is resumed. Despite its widespread use throughout the fire service and continued enhancements in recent years, the PASS device is not perfect. Prior to this study, minimal scientific research had been performed to determine optimal PASS characteristics, and several questions about the device's efficacy exist. Can the alarm be clearly heard over ambient fireground noise? Is the content of the alarm optimized for localizing the source as quickly as possible? Is the alarm capable of penetrating complex structure geometries to provide a useful signal at necessary distances? This work seeks to improve firefighter safety by answering these questions and applying the answers to device optimization throughout the fire service. 


\section{$1.1 \quad$ Literature Review}

On May 12, 1979, Engineer Lynn Hazlitt of the Los Angeles Fire Department Squad 39 became disoriented while fighting a structure fire in a large industrial warehouse [1]. Firefighters on the scene heard his shouts for help and knew that Hazlitt needed assistance but did not know where to begin searching in the large, smoke-filled building. One firefighter managed to find Hazlitt but was forced to leave him when his air supply ran out. A false report that Hazlitt had been rescued delayed further search efforts. His body was discovered and identified after the fire.

Hazlitt's death, which was one of several firefighter fatalities around that time where the downed firefighter could not be located [2], instigated the development of the PASS warning device.

After a departmental analysis of Hazlitt's death, the International Association of Fire Fighters (IAFF) affiliate, Local 112, requested that the California Occupational Safety and Health Administration (CAL/OSHA) investigate the incident [3]. Their report was released on November 1, 1979. The chief investigator said, "It is my belief that the fire fighters need some type of warning devices as a method to alert fellow fire fighters if entering a smoke-filled building. It is a device that would enhance their survival tremendously." [4] The investigation mandated that all LAFD firefighters wear and use PASS devices, despite reluctance from city fire officials. The officials' reluctance led to the comprehensive testing necessary for the first iteration of a standard for designing and testing PASS devices. 
There have been many iterations of PASS since its inception in 1982 , when the first standard was released by the National Fire Protection Association (NFPA). The first versions of the alarm were autonomous from the rest of the fireman's personal protective equipment (PPE). The device was clipped onto the firefighter's uniform or equipment and manually switched to an "On" status. However, the device was prone to failure simply because many firefighters forgot to activate the device prior to entering a dangerous area [5]. Additional problems included a high rate of false alarms, breakage, and a signal easily confused with smoke detectors and alarm horns.

Newer versions of the NFPA standard have been released since the original in 1982, and great strides have been made in defining the design and operation of PASS devices, each addressing issues to improve the PASS device as it becomes increasingly engrained in the PPE of firefighters nationwide.

For example, prior to 2007 NFPA 1982, Standard on Personal Alert Safety Systems (PASS), the PASS device was not required to be integrated into the self-contained breathing apparatus (SCBA), and fire chiefs in major departments nationwide noted firefighters' failure to activate their devices before entering the fireground. On April 11, 1994, two Memphis, Tennessee firefighters died in the line of duty while fighting a well-developed fire in a highrise apartment building. One firefighter was found entangled in wires near the stairwell entrance on the fire floor; the other was found in an apartment on the fire floor that did not burn but was filled with toxic smoke and gases. Their manually operated PASS devices were not activated [6]. On February 
14, 1995, three Pittsburgh, Pennsylvania firefighters died in the line of duty while fighting an arson fire in a single-family dwelling. All three firefighters were wearing PASS devices, yet none of the devices were activated [7].

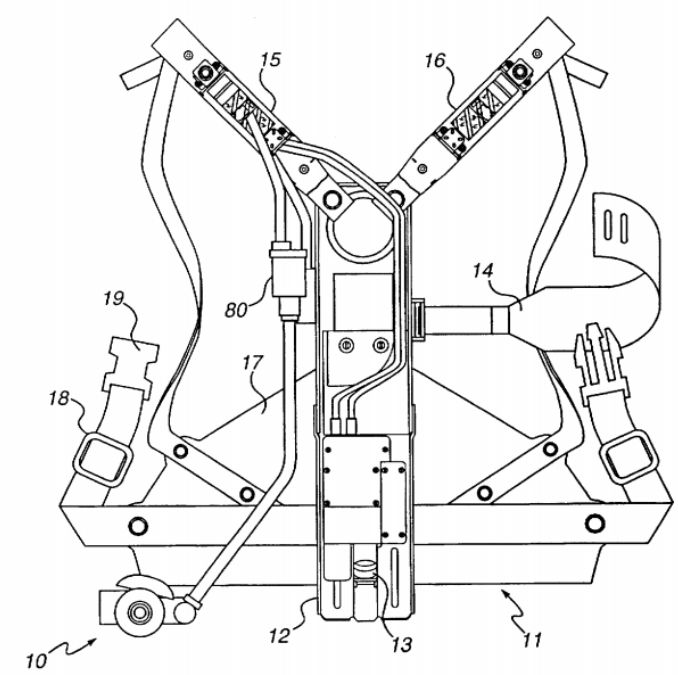

Figure 1.1: Front perspective view of an MSA SCBA, where 10 is the integrated PASS device. Adapted from [8].

The NFPA responded to these and other similar fatalities by pushing manufacturers to (and eventually enforcing with the 2007 standard) begin integrating the PASS device with the SCBA. Several failed alternatives were attempted, including one version where the PASS device was integrated into a pressure sensor connected to the air chamber of the SCBA [9]. However, this added unnecessary complexity to operations as the switch had to be changed out each time the compressed air bottle was changed. It was also prone to functional failures. Individual patents for modern PASS devices vary by manufacturer. Generally, these alarms are activated when the knob on the air 
cylinder is turned. Additionally, single-motion manual activation, such as the capability to press a single button to place the PASS in full alarm, is a requirement of the standard. A pre-alert is included to alert active firefighters to a lack of motion and prevent false alarms.

\subsubsection{Line of Duty Death Reports}

In the scope of the PASS Audibility Study, a review and analysis of contemporary Line of Duty Death reports, released annually by the National Institute of Occupational Safety and Health (NIOSH), was undertaken [10]. In total, 73 reports from 1998 to 2012 were analyzed. Only cases involving or mentioning a PASS alarm were included. In $30 \%$ of these cases, the PASS alarm was heard and localized. In these cases, the victims generally died of smoke inhalation or thermal inhalation before localization could occur. In $16 \%$ of the cases surveyed, the PASS device had excessive heat damage that prevented it from alarming property. In another 9\%, the PASS was confirmed

functional, but the reason for failure was unknown. These failures are outside the scope of our improvement study.

The areas that form the basis for this report account for approximately $23 \%$ of the cases surveyed. In these cases, either background noise prevented rescuers from finding the downed firefighter, the PASS alarm was muffled by debris or the firefighter's body positioning, or the PASS was heard but was unable to be localized.

One such example occurred in July of 2010, two firefighters were found 
unresponsive at a residential structure fire. Both PASS devices' alarms were sounding when they were discovered, but the firefighters had asphyxiated in the search time [11]. In 2011, a firefighter died during fire-fighting operations on the 2nd floor of a three-story apartment building. Several firefighters stated that they heard a PASS alarm sounding, but were unable to determine the location [12]. In 2012, a firefighter was killed at a theatre fire after the roof collapsed, trapping him within the theatre. Firefighters commented that the noise in the collapse area was extremely loud. One firefighter stated that "he heard what he thought was a PASS device alarm, but he was not sure due to the noise of the PPV fans running and the elevated master stream flowing water into the building [13]."

Such fatalities, where an optimized PASS device might have eliminated the death, is the focus of this work, to be embodied in future iterations of PASS design.

\subsection{PASS Audibility Study}

The PASS Audibility Study was funded by the Federal Emergency Management Agency (FEMA) in 2010, with the backing of the Fire Protection Research Foundation (FPRF). The project aimed to directly support firefighter safety by providing science-based guidance to PASS device manufacturers,

researchers, and standards-developing organizations for the optimization of PASS alarm sounds. It also aimed to improve search-and-rescue training protocols for firefighters. The specific objectives for meeting these goals were to 
1. Provide science-based guidance to PASS device manufacturers, firefighters, researchers, and standard-developing organizations for the optimization of PASS alarm sounds

2. Investigate the feasibility of technological enhancements to PASS devices that can be implemented within five years

3. Produce a methodology by which to optimize audible alarms that can be applied to a wide range of research areas

The project team analyzed the fireground environment using the passive sonar equation commonly employed in underwater acoustic analyses [15]. In such a system, the source signal, the transmission through the medium, the receiver and the noise levels must be defined. This information is used in an energy balance known as the passive sonar equation,

$$
S L-T L=N L-D I+D T,
$$

where $S L$ is the signal level, $T L$ is the transmission loss through the medium, $N L$ is the noise level in the environment at the receiver, $D I$ is the directivity index associated with the receiver, and $D T$ is the detection threshold of the receiver. By analyzing and optimizing each of the components of the passive sonar equation, the component of interest (in this case, the detection threshold of the receiver) can be improved. 


\subsection{Overview of Work Performed}

Previous work on the experiment aimed to analyze each component of the passive sonar equation. The PASS device itself has been analyzed and measured to define the signal level. A study of different noise sources on the fireground, including saws and ventilation fans, was performed, and a similar study analyzing the noise of the fire itself was performed. Both the detection threshold and directivity index have been analyzed in audiology studies addressing how the PPE affects the firefighters ability to hear. The primary research focus areas are outlined in more detail in the following sections.

\subsubsection{Acoustical Analysis of Firefighting Equipment}

Much work has gone into quantifying the acoustic effects of firefighting equipment on the fireground [16, 17]. In the passive sonar equation, Equation (1.1), this analysis quantifies the signal level (SL) and noise level (NL) on the fireground. The equipment measured includes engine noise, chain saws, circular saws, positive pressure ventilation (PPV) fans, and the PASS device itself. This research independently verifies extensive research performed by NIOSH in the last several decades in conjunction with the formulation of hearing dose and noise exposure criteria.

Engine noise has been a topic of particular concern and the focus of several NIOSH Health Hazard Evaluations (HHE). Engine noise as a source of occupational noise exposure was first brought to attention by researchers at the University of California, Irvine in 1979. They recorded sound pressure levels 


\section{Figure 4}

One-Third Octave Band Sound Levels

1976 Pirsch Diesel Engine \#5

HAMILTON FIRE DEPARTMENT

HETA 89-0026
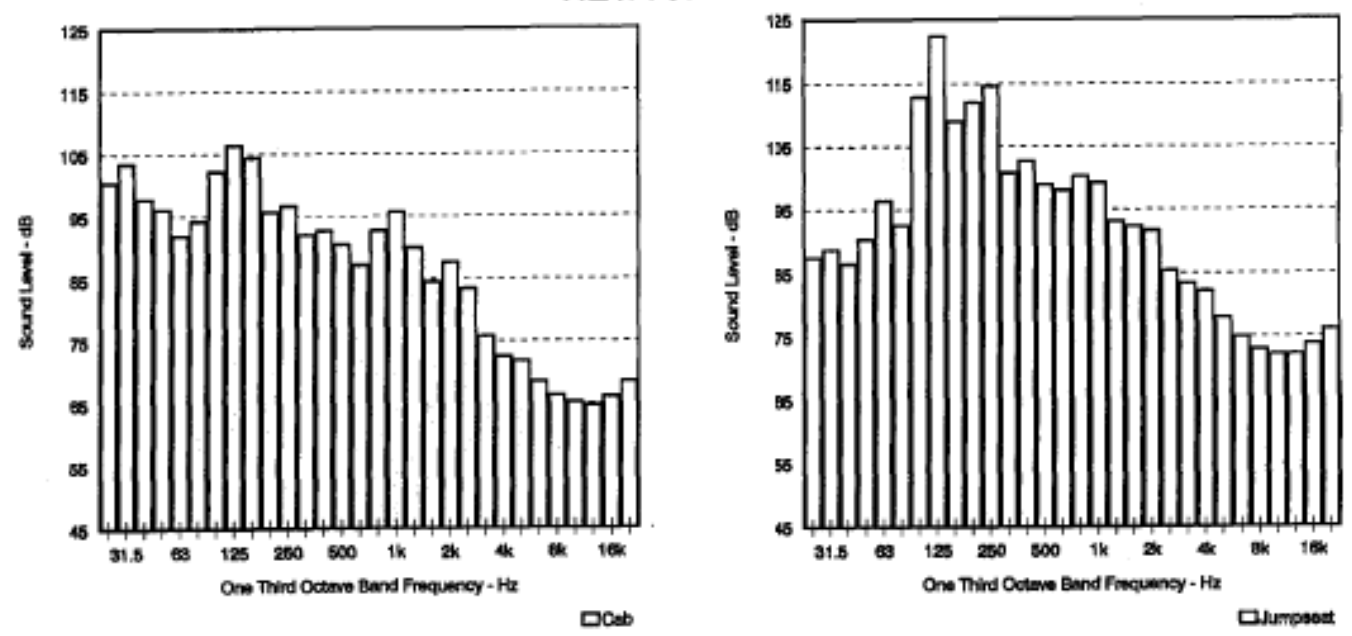

Figure 1.2: NIOSH measured levels for specific fire vehicle. Adapted from [18].

in excess of $115 \mathrm{dBA}$ [19]. In 1982, the first related NIOSH HHE examined truck and siren noise levels in the Newburgh Fire Department, with some intermittent noise levels reaching between 100-116 dBA [20]. A similar study was requested in August of 1981 by the Fire Department of the City of New York. They found engine and siren noise levels ranging from 80-115 dBA [21]. In October 1988, an administrator of the Hamilton, Ohio Fire Department requested the measurement of noise exposure levels emitted by various fire vehicles [18]. Spectral analyses revealed noise levels in excess of 120 decibels at certain frequencies as shown in Figure 1.2. 
The present research team performed similar measurements on engines without an active siren, as would typically be found on a fire scene, according to ANSI testing specifications [22]. Engine operating conditions varied depending on whether the pump and generator were operating in addition to the ambient engine noise, and the signal level varied accordingly from approximately 85$90 \mathrm{~dB}$. Because the siren was not included, these levels were lower than the previously-described recorded values. A variety of other equipment was also analyzed, including saws and ventilation devices. The measured levels are roughly in accordance with those measured by Neitzel et.al. in 2012 [23]. The measured levels were filtered into third-octave bands [24], A-weighted [25], and compared to the levels of the PASS device at equal frequency bands. They were also compared at four different positions angularly around the device. These measurements are of importance in the modeling work described in later sections.

\subsubsection{Modeling and Validation of Sound Transmission}

Transmission loss is another important component in the passive sonar equation. Anecdotal evidence from fireground personnel suggests that sound transmission might be altered in a fire-affected space.

Sound transmission is dependent on the speed of sound, density, and acoustic impedance of air, which are in turn dependent on temperature. One source of these changes in sound transmission comes from large temperature distributions within the space. The acoustic impedance mismatch between 
different temperature levels causes an interface where reflections will occur. Acoustic impedance describes the opposition a fluid presents to an acoustic flow, and the characteristic impedance is the acoustic impedance for a plane wave in that fluid [26]. The characteristic impedance $Z_{0}$ is determined by material properties of the fluid, density $\rho_{0}$ and sound speed $c_{0}$ (Eq. (1.2)), where $c_{0}$ and $\rho_{0}$ are functions of temperature (Eq. (1.3)):

$$
\begin{gathered}
Z_{0}=\rho_{0} c_{0}, \\
c_{0} \equiv \sqrt{\gamma R T} .
\end{gathered}
$$

The amount of sound pressure reflected at a given interface (i.e. between two temperature points) is defined by the reflection coefficient, as defined in Equation (4.14):

$$
R=\frac{Z_{2}-Z_{1}}{Z_{2}+Z_{1}}
$$

In a room with constant temperature, reflection will only occur at room boundaries. As the temperature increases at the top of the room, however, sound speed will increase and density will decrease, and the characteristic impedance of that air will change and create an interface lower than the physical room boundary. This would change the sound transmission through a room and signal perception at the receiver.

As a continuation to the idea of sound reflection, ray theory states that for a stratified or continuously varying temperature (and therefore stratified 
or continuously varying sound speed), a wave will deviate from a straight line in a process known as refraction [27]. The fundamental idea behind refraction is Snell's Law, in which the angle of reflection for a wave is defined by the ratio of sound speeds between the two mediums:

$$
\frac{\cos \theta_{1}}{c_{1}}=\frac{\cos \theta_{2}}{c_{2}},
$$

where $\theta_{1}$ is the angle of incidence of the ray onto the interface and $\theta_{2}$ is the angle of transmission. This idea can be repeated for a multilayer stratified medium. For a continuously varying medium, a differential form of Snell's law is required (Eq. (1.6)):

$$
\frac{d c}{d \theta}=-\frac{c \sin \theta}{\cos \theta}
$$

As the change in reflection and transmission angles evolve through the continuum, the ray begins curving through the medium, as demonstrated by the sound transmission paths in Figure 1.3. The reflection and refraction effects caused by the fire demonstrate numerically how a fire could change sound transmission in a room.

In order to better understand the fire's effect on an audible alarm, a recording of the PASS alarm was played and recorded inside the burn facility at the University of Texas. In addition to the qualitative evidence gained from listening to the recording, the research team was able to characterize the changes in impulse response in the room at various stages of fire growth. A 

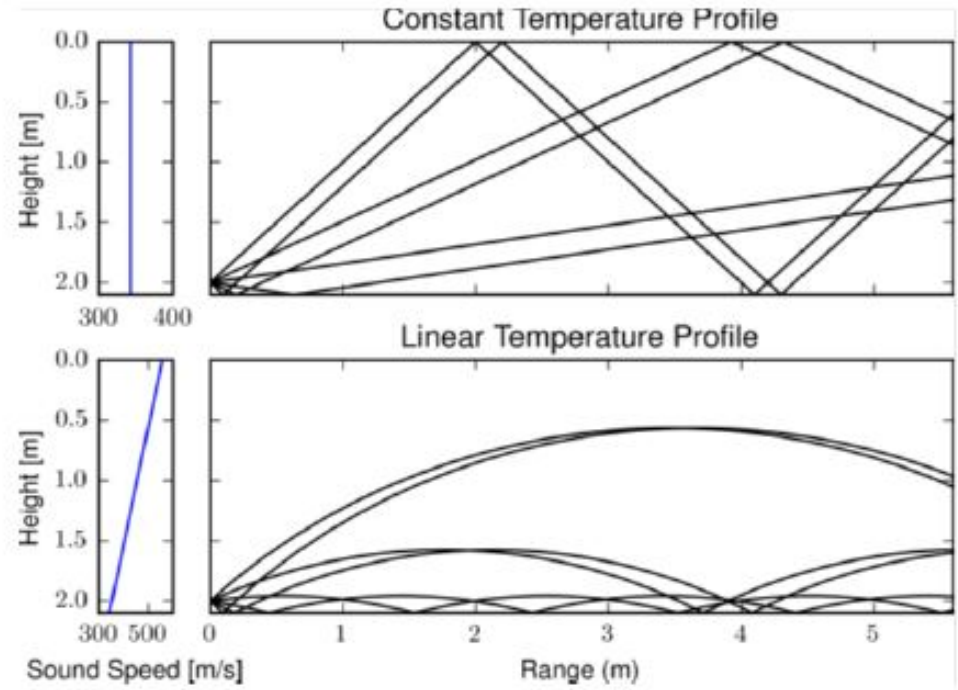

Figure 1.3: Example of sound refraction in the presence of a sound speed gradient. Adapted from [14].

spectrogram of this test is shown in Figure 1.4. At $t=0$, an audio sample of the PASS alarm was played with no fire present, and the spectrogram shows the impulse response of the room alone. At ignition, the low frequency room modes increased in frequency, and the high frequency modes were attenuated. This experiment was further validated numerically using a computational and fluid dynamics software package named Fire Dynamics Simulator (FDS) and a finite element software (COMSOL). The fire causes a noticeable change for the listener and has the potential to make the PASS alarm less audible to searchers on a fire scene. 


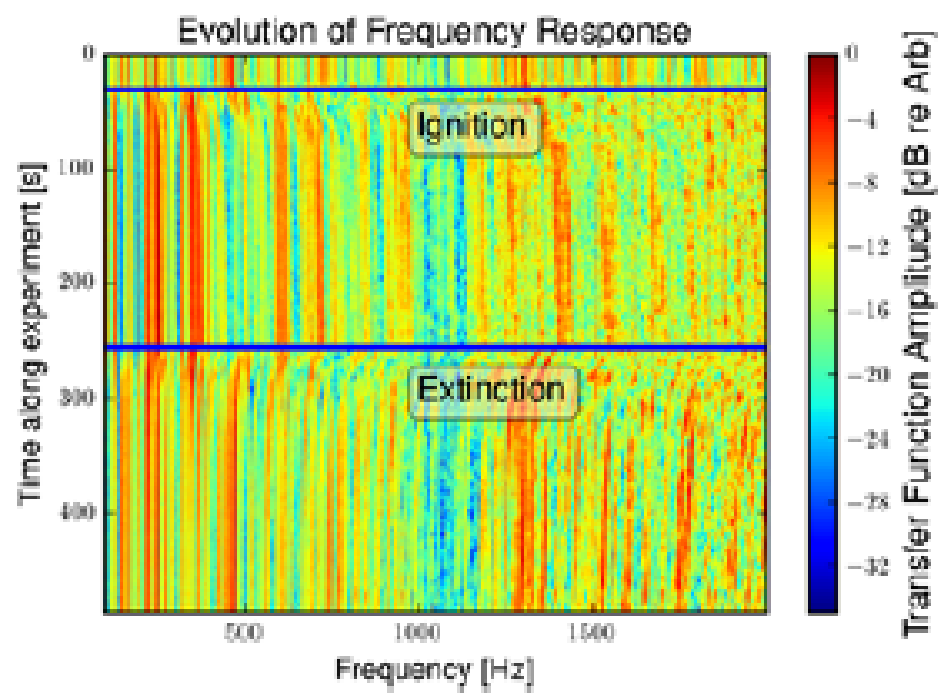

Figure 1.4: Example of changing transfer function during a fire. Adapted from [28].

\subsubsection{Physiological Acoustics and Audiology Testing}

The last major component of the PASS audibility study determined how firefighter PPE affects their detection threshold and ability to localize sound. A lot of work has been done in the military sector to quantify the effect of helmets and uniforms affect a person's ability to detect and localize sound $[29,30]$. Military researchers have tried to optimize their helmets, but some of their solutions, such as reductions in ear coverage, would prove to be more difficult to implement in the fire service.

The PASS Audibility Study aimed to quantify the effect of PPE specifically for firefighters. These studies were undertaken in two major phases one using an acoustic manikin and another using human subjects, roughly 
following the methodology outlined by Li in 2009 [31]. Initial work indicated that the helmet had the greatest impact on the listener head-related transfer function (HRTF), so research efforts were focused on the effect of the helmet. Eleven different helmets were acquired and tested, of traditional, modern, and European style. In the first two cases, the ear is exposed; in the latter case, the ears are covered by the hard shell. Based on the manikin studies, the use of firefighter PPE will alter the signal reaching the ears of firefighters [16]. On average, the helmet lowers the received level by $3 \mathrm{~dB}$ and adds artifacts that will potentially alter the firefighter's ability to localize sound.

Additionally, an audiology study was performed to show how PPE affects the firefighter's auditory threshold of the sound. Subjects were tested using a range of tones and directly with the PASS signal itself. The results of these studies indicate that firefighter PPE causes a mean detection threshold shift of about $7 \mathrm{~dB}[16]$. In the low frequencies, there was no significant difference; in the 3-5 kHz range and for the PASS signal, however, the threshold tended to drop from 5-15 dB depending on the subject.

\subsubsection{Conclusion}

The previous work on the PASS Audibility Study attempted to identify how each individual component of the passive sonar equation formalism contributes to the identification and localization of the PASS alarm on the fireground. Detection and localization are affected by source characteristics of the PASS device itself, the presence and characteristics of masking noise sources on 
the fireground, the physics of sound transmission within the structure during a fire, and the insulating effects of the PPE on the receiver.

To optimize the PASS device, all of the prior work needed to by synthesized into a more comprehensive test to identify actual improvements to support firefighter safety. Realistic search-and-rescue training exercises were used as a compendious study of the PASS device in action and are described thoroughly in the following section. 


\section{Chapter 2}

\section{PASS Localization Tests}

\subsection{Background}

Fire departments across the United States are interested in the effects of noise on firefighter search and rescue tactics. In 2014, the Yakima Fire Department Training Division (YFDTD) performed a series of drills to attempt to emulate a commercial fire that led to a firefighter fatality in Phoenix, Arizona [32]. In the drills, blackout conditions were recreated by blacking out face pieces and filling the space with smoke from smoke machines. Noise sources, including electric portable speakers, smoke detectors, generators, and charged hoselines were strategically placed throughout the building to create audio interference and external noise.

The conclusions from these tests strongly verified the necessity for work performed by the Fire Research Group at the University of Texas (UTFRG). The YFDTD's primary conclusion was that the PASS device was failing; firefighters either forgot to stop to listen for the alarm, could not differentiate the alarm from smoke detectors, or mistook the alarm for an accidental activation by one of their own crew members. Additionally, the presence of excessive radio communication led to the inability of crews to hear or listen for PASS 
devices. One solution that they suggested was the incorporation of PASS device alarms and noise into search and rescue drills.

The UTFRG performed similar tests to synthesize all of the prior work into a comprehensive look at the way noise acts as a stressor during search and rescue scenarios [33]. The results will help determine how to translate the study's preliminary work into device optimization and how to improve training protocols to integrate the presence of noise in search-and-rescue training drills.

\subsection{Methodology}

The UTFRG performed four different experiments at three fire departments across the United States. Two were performed at the Austin Fire Department (AFD), one was performed at the Glendale Fire Department (GFD), and one was performed at the Oklahoma City Fire Department (OKCFD). The

firefighters were instructed to perform a crawling search in full PPE (coat, hood, helmet, etc.) until they could identify the location of a PASS signal in a structure. They were instructed to double tap their helmet when they first identified the PASS signal. Their vision was obscured, as in the YFDTD tests, by occluding the face mask with crumpled wax paper. The tests were run for either twelve or fourteen firefighters. 


\subsubsection{Scenarios}

For each field test, the research team predetermined the location of four background speakers ${ }^{1}$, one PASS speaker ${ }^{2}$, and the firefighters' search starting point. Each site had two unique PASS locations - one "long" pathlength and one "short" pathlength. The background speaker locations remained constant for each PASS location. The sources of background noise were located in positions identified to most complicate the localization task. Background noise was provided by previously recorded sounds including two chainsaws, a positive pressure ventilation fan, and an engine running with pump and generator. The levels were adjusted based on the results of the acoustic analysis of firefighting equipment (see Section 1.3.1) to best imitate the actual equipment.

At each testing location, the firefighters were split into two groups. They alternated so that each firefighter went through the test once with background noise and once without noise. Additionally, the firefighters alternated so each went once through the "long" course and once through the "short" course.

\subsubsection{Test Layouts}

Each fire department was asked to provide a testing facility for the field tests. Once a location was agreed upon, the fire departments provided a floorplan of the building so that the speaker locations could be decided, as

\footnotetext{
${ }^{1}$ Four JBL LSR2328P speakers were used as background noise sources.

${ }^{2}$ A KRK Rokit 5 G3 speaker was used to produce the PASS alarm.
} 


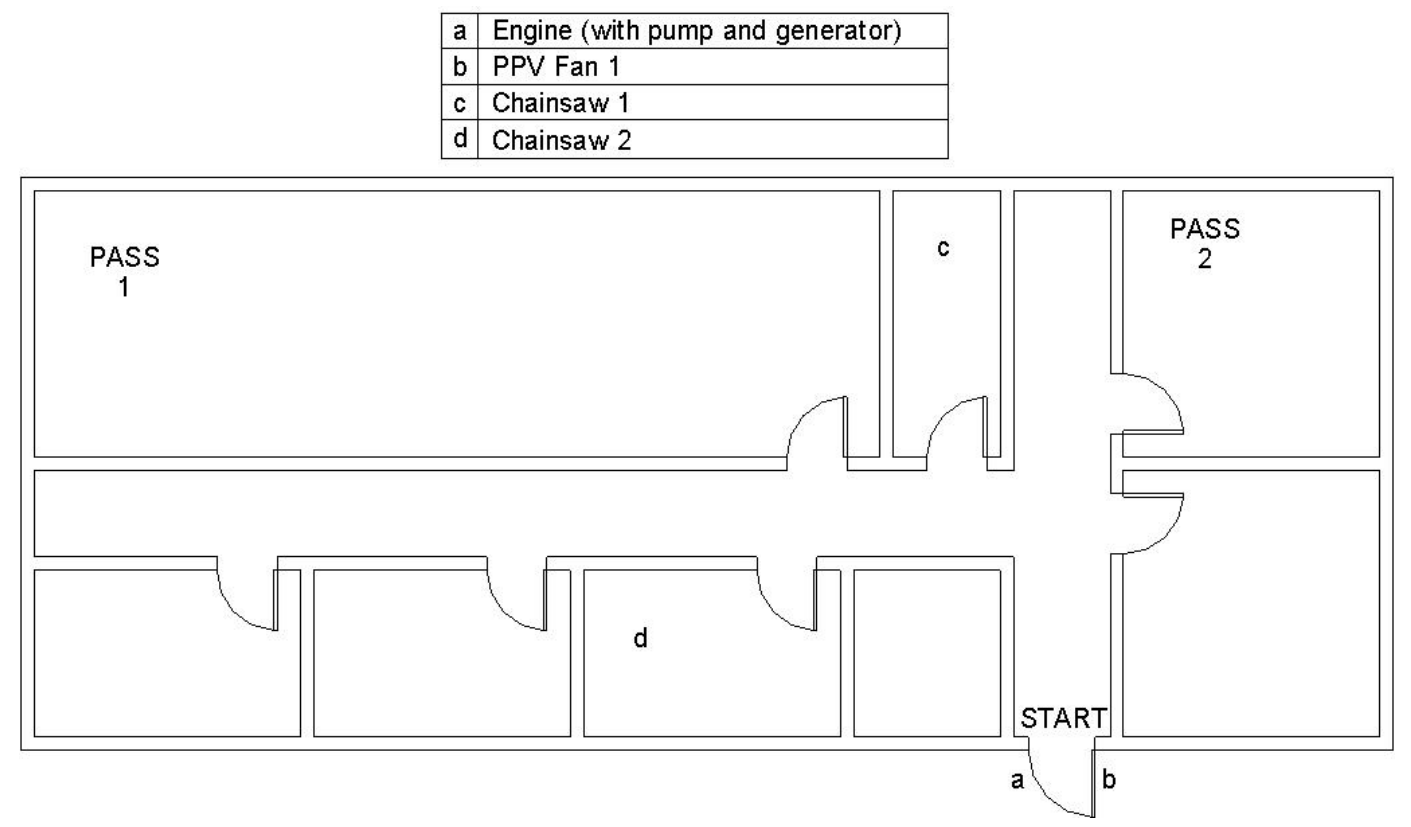

Figure 2.1: The floorplan of the AFD portable office building with the location of relevant sound sources.

described above.

\subsubsection{Austin Fire Department}

The Austin Fire Department provided a portable office building at the AFD training facility. The building has two hallways and seven rooms representing approximately $1780 \mathrm{sq} \mathrm{ft}$. The ceiling is a typical height for an office building (approximately $10 \mathrm{ft}$ ). Linoleum covers the floor of the hallway and four rooms, and the rest is carpeted. The walls throughout are a standard gypsum construction. The layout of the building is shown in Figure 2.1.

Two scenarios were identified in this building. In the long pathlength 
scenario, the PASS speaker was located at the end of the large room furthest

from the entrance. It is identified in Figure 2.1 by "PASS 1". The direct path length from "START" to "PASS 1" is approximately $80 \mathrm{ft}$. In the short pathlength scenario, the PASS speaker was located against the back wall of the office in the top right corner of the building. It is identified in Figure 2.1 by "PASS 2". The direct path length from "START" to "PASS 2" is approximately $24.5 \mathrm{ft}$. The noise sources are identified in Figure 2.1 by icons and are defined in the legend.

It is also relevant to note that the building floor was not perfectly clear, as shown in the floorplan. Chairs, desks, and training mats were scattered throughout the building. The "PASS 2" speaker, for example, was placed between a desk and the wall. These obstacles are not shown here.

\subsubsection{Glendale Fire Department}

The Glendale Fire Department provided a warehouse space at the GFD training facility. The warehouse, though only part of the total training facility building, is approximately $9500 \mathrm{sq} \mathrm{ft}$. The ceiling is $40 \mathrm{ft}$ high, although a catwalk lowers the effective ceiling in part of the warehouse to approximately $25 \mathrm{ft}$. The majority of the wall and floor space is covered in either concrete or CMU. The layout of the building is shown in Figure 2.2.

In the middle of the warehouse, several large training obstacles could not be moved. These were used to partially obscure the PASS source, much like the desk was used to obscure the "PASS 2" speaker in the AFD tests. 

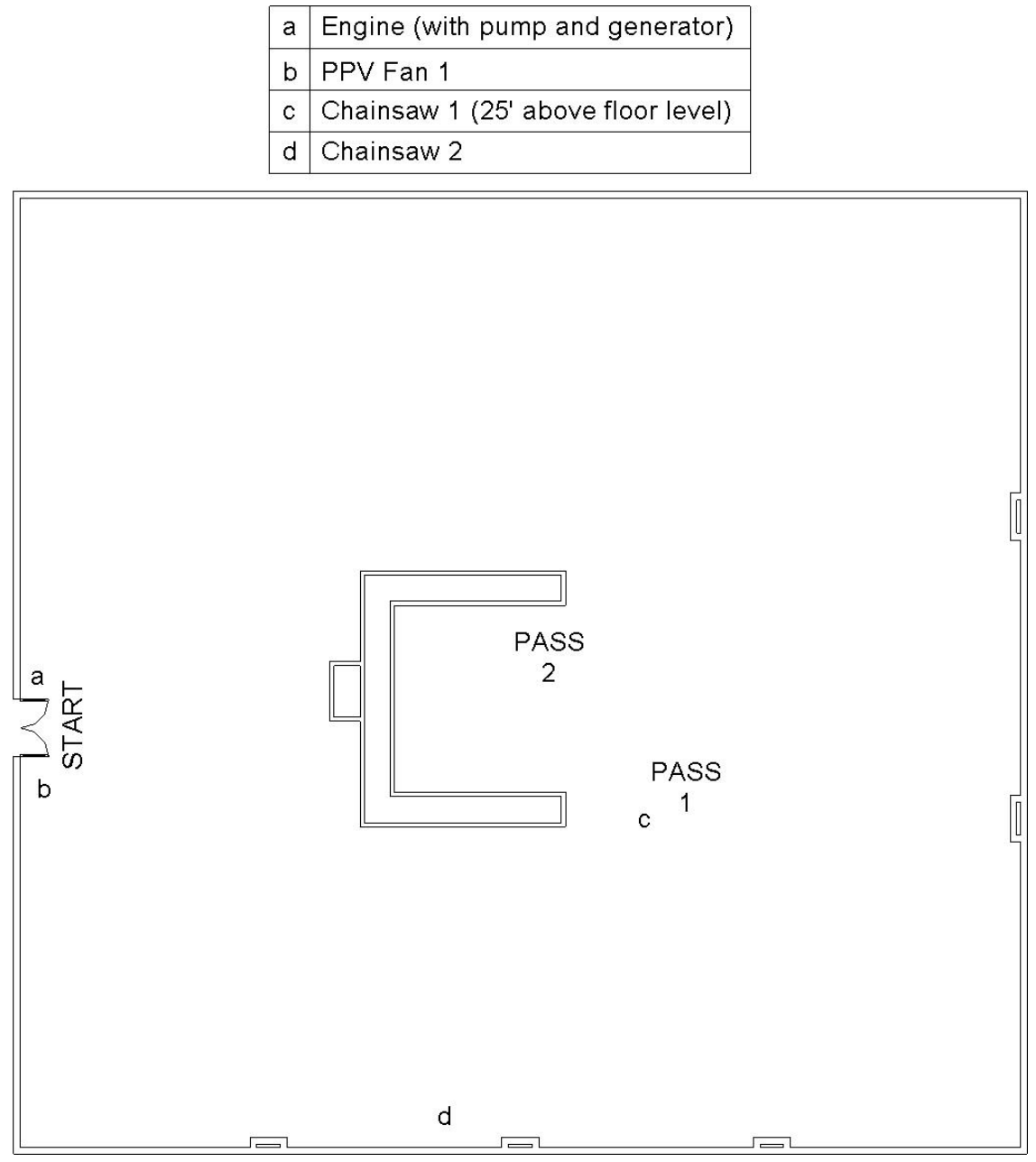

Figure 2.2: The floorplan of the GFD warehouse space with the location of relevant sound sources. 
Two scenarios were identified in this building. In the long pathlength scenario, the PASS speaker in the right section of the warehouse. It is identified in Figure 2.2 by "PASS 1". The direct path length from "START" to "PASS 1" is approximately $79.5 \mathrm{ft}$. In the short pathlength scenario, the PASS speaker was located directly against the training obstacle in the right-center of the building. It is identified in Figure 2.2 by "PASS 2". The direct path length from "START" to "PASS 2" is approximately $62 \mathrm{ft}$. The noise sources are identified in Figure 2.2 by icons and are defined in the legend. The first chainsaw and the "PASS 1" speaker overlap on the floorplan; the chainsaw speaker was placed on the catwalk and pointed down towards the "PASS 1" speaker.

This warehouse setting provides an interesting foil to the office space setting used in the AFD tests. It has more reflective surfaces and more indirect sound arriving at the "START" position, which should make localization more difficult $[34,35]$.

\subsubsection{Oklahoma City Fire Department}

The Oklahoma City Fire Department provided a combined warehouse/office space at the OKCFD training facility. The research team opted to use the warehouse space as a staging area for the firefighters and perform the search test in the office space, covering approximately $2548 \mathrm{sq} \mathrm{ft}$. The office space had standard ceiling heights throughout (approximately $10 \mathrm{ft}$ ). The floor

throughout is concrete and the walls are a CMU construction. The layout of 

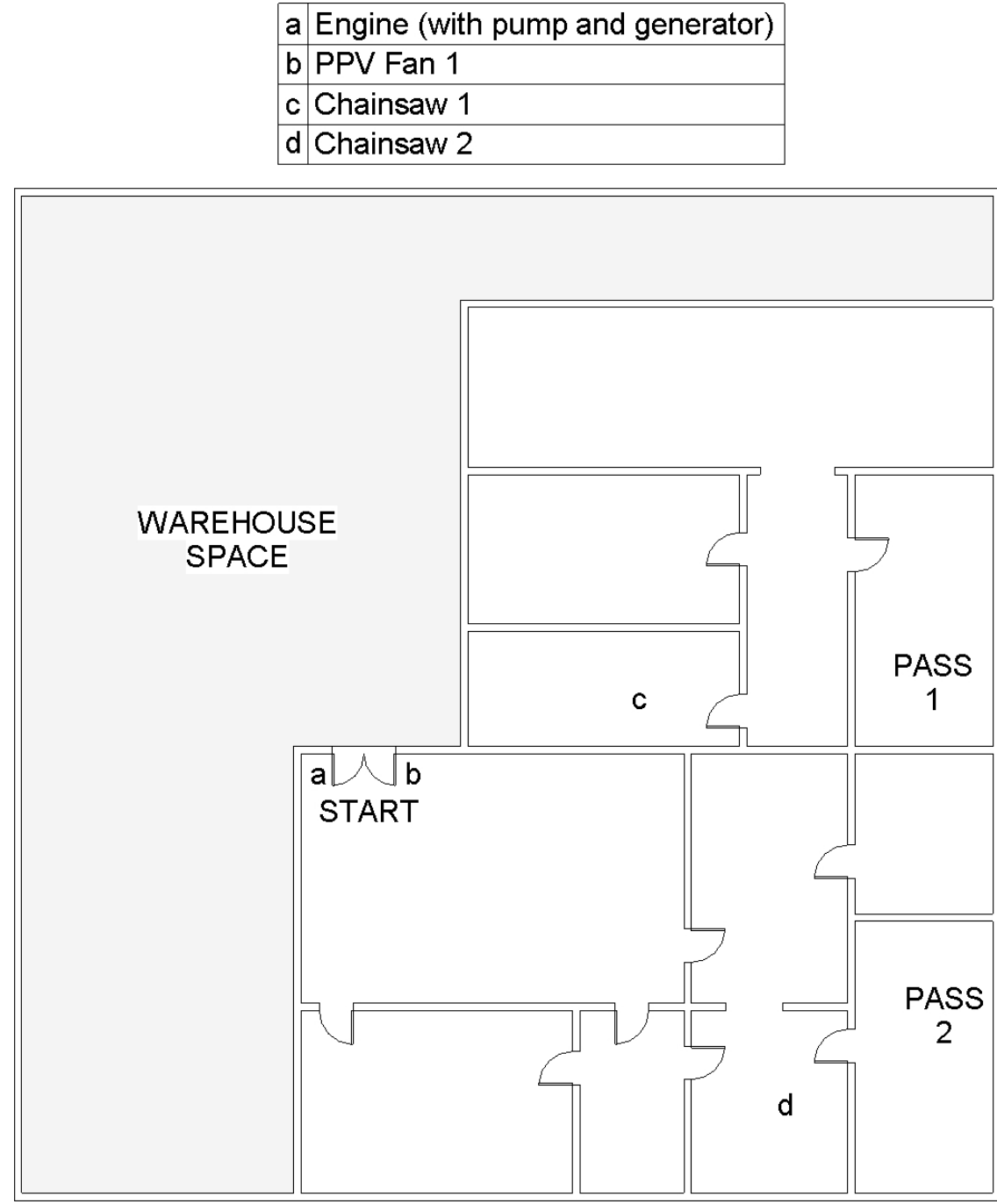

Figure 2.3: The floorplan of the OKCFD warehouse space with the location of relevant sound sources. 
the building is shown in Figure 2.3.

Two scenarios were identified in this building. In the long pathlength scenario, the PASS speaker in an office near the far right wall of the building. It is identified in Figure 2.3 by "PASS 1". The direct path length from "START" to "PASS 1" is approximately $62.7 \mathrm{ft}$. In the short pathlength scenario, the PASS speaker was located in the bottom right office. It is identified in Figure 2.3 by "PASS 2". The direct path length from "START" to "PASS 2" is approximately $54.5 \mathrm{ft}$. The noise sources are identified in Figure 2.3 by icons and are defined in the legend.

As in the AFD office building, obstacles were present on the floor of the building. Chairs, desks, and various amounts of debris were present from practice burns performed by the OKCFD training unit. There were also two bathrooms with stall doors dividing the space. These obstacles are not noted in Figure 2.3.

\subsection{Summary of Results}

Several metrics were extracted to attempt to quantify the information gained from these localization tests. The measured completion times for each iteration (i.e. for each firefighter and each scenario) were compiled to obtain a statistical overview of the data, including the mean, standard deviation and cumulative distribution. These measurements showed predictable results. The firefighters took more time to find the PASS speaker located further away. In all cases, the addition of noise also significantly increased the time to identifi- 
cation. This effect, as expected, was most identifiable in the long pathlength scenarios.

Another metric recorded was the average firefighter obscured vision crawling speed. One consequence of the presence of noise was that the firefighter crawl path was rarely straight enough to obtain an obvious crawling speed. Without the noise, however, the average crawling speed was approximately $1 \mathrm{ft} / \mathrm{s}$. Nagai et.al. found an average speed of $0.73 \mathrm{~m} / \mathrm{s}$ for a single civilian crawler (approximately $2.4 \mathrm{ft} / \mathrm{s}$ ) [36]. As expected, the firefighters crawled significantly slower than the civilians due to obscured vision and the presence of noise. Additionally, the detection and localization task required them to routinely stop and listen, which also decreased the average crawling speed. Crawling training exercises are present in most major fire department training programs [37], but the inclusion of noise in training could also help improve search-and-rescue times.

\subsubsection{Need for Masking Visualization}

There are two primary motivations to develop a simple, relatively fast method to map the sound field from the PASS alarm and the various sound sources.

The first motivation is to standardize the process of placing the sound sources in the preplanning of each test scenario.

The second motivation is to identify tricky situations that might be used to expose firefighters to acoustic effects that they would not otherwise 
be aware of. For example, for the "PASS 1" location in the AFD office space, multipath reflections caused some searchers to sense the presence of the alarm in the top right room. Several firefighters became disoriented, entering the room and refusing to leave, convinced that the alarm was sounding in that space. One firefighter circled the room until he ran out of air and researchers were forced to stop the test, a full 16 minutes after entering the space, as shown in Figure 2.4.

An acoustic model, simple enough to be understood by a nontechnical audience, could help convey these unexpected effects to firefighters and better prepare them for such events in actual search-and-rescue scenarios. The methodology determined to best model the localization test acoustics is described in the following section. 


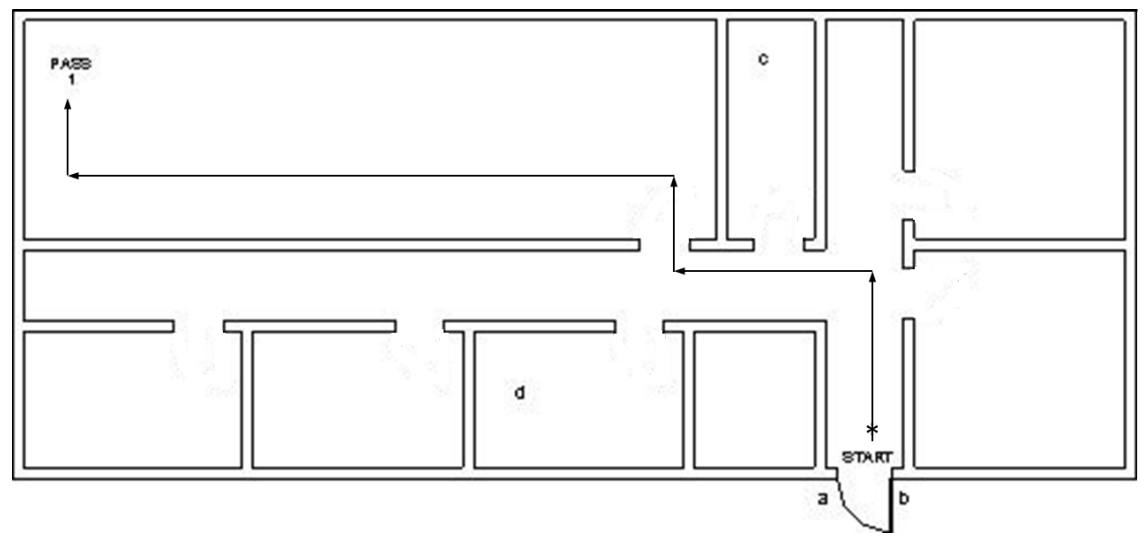

(a) Sample Path Without Background Noise

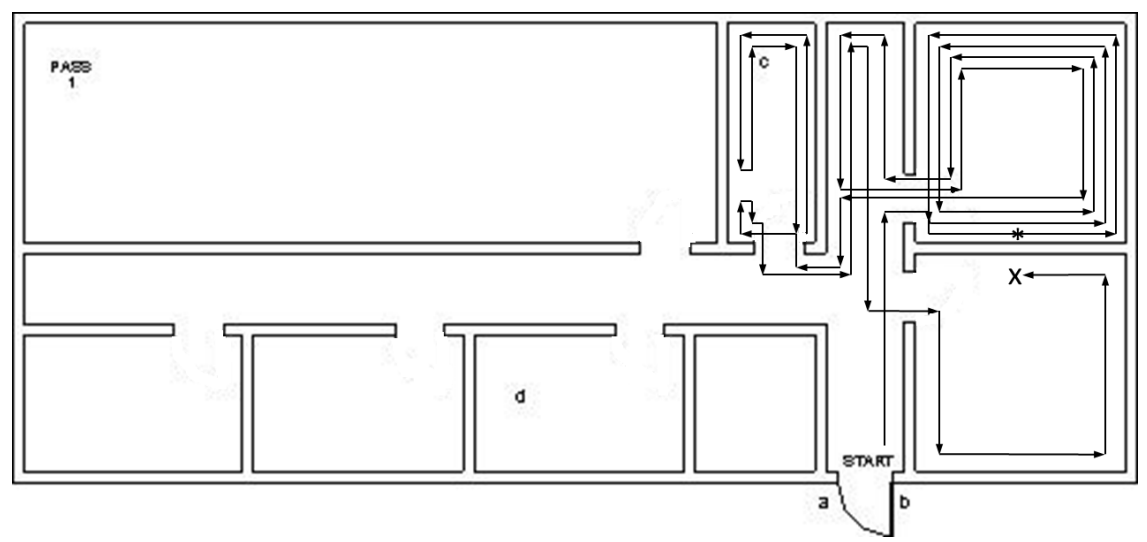

(b) Sample Path With Background Noise

Figure 2.4: Sample paths with and without background noise. The asterisk represents where the firefighter first alerted to audible detection of the alarm signal. 


\section{Chapter 3}

\section{Auralization Mapping}

\subsection{Methodology}

\subsubsection{Ray Tracing}

A common acoustic modeling method is known as ray tracing [38]. A surface model of an enclosed space is created by a mesh of contiguous polygons, where each polygon is defined by its edges, vertices, a normal, and material properties (including absorption and diffusion coefficients). A finite number of rays are cast mathematically from a user-defined source; the ray tracing program tracks the original source strength and location, directivity, direction, total distance from the source, amplitude, and bounds of each ray.

A receiver is modeled as a point or sphere within the enclosed space. In ray tracing, a receiver hit occurs when a ray passes within a given radius of the receiver. If no receiver hit occurs, the ray is followed until it encounters a surface. When there is a surface hit, reflection calculations are performed based on the impact point using the surface normal, area, and material properties.

The output of a ray-tracing program includes the room's impulse response at the point of a given receiver. The analysis must be repeated for each individual reception point to obtain impulse responses throughout the space. 
The research team attempted to use a commercially-available ray tracing software, Autodesk Ecotect, to create an acoustic model for early presentations to firefighters. Ecotect was one of the first commercial sustainability tools to include an acoustic analysis component, aimed at architects and other building designers [39]. An image of the Ecotect ray tracing output is shown in Figure 3.1.

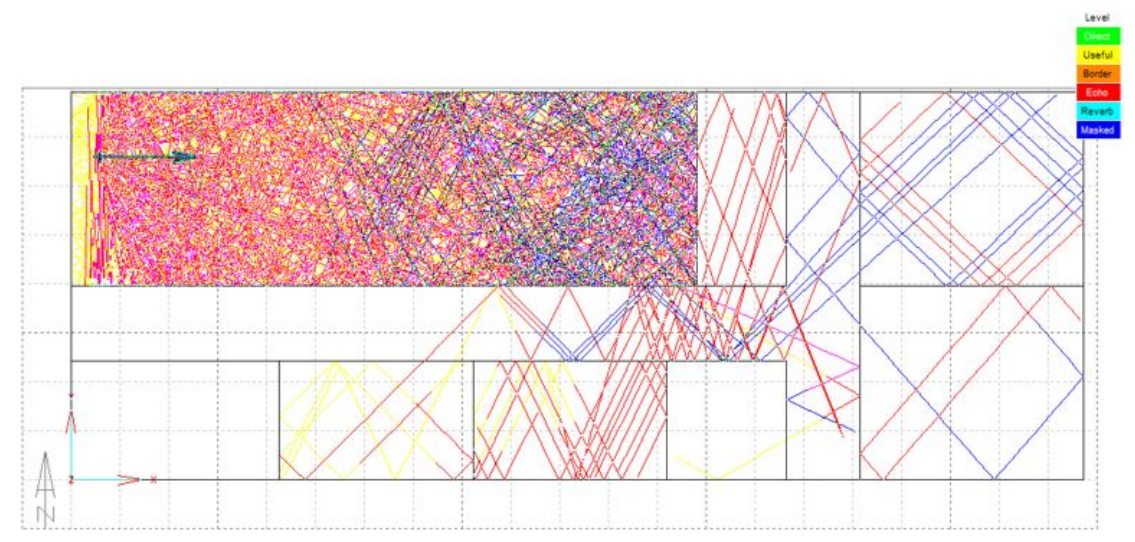

Figure 3.1: Ecotect ray tracing example in AFD office building.

Ecotect is very limited in its analysis capabilities. It does not compute an impulse response geometrically, like most ray tracing softwares. It instead uses a statistical method, subtracting a specified amount of decibels per reflection for each ray. The software then compiles all of the decaying rays to give you the reverberation time (when the overall sound level has decreased by 60 $\mathrm{dB}$ ). This provides an idea of the impulse response for the room, but will not give you a location-specific impulse response.

Even with a more technically-advanced software, ray tracing has draw- 
backs that make it unsuitable for the modeling challenge at hand. Ray tracing methods rely on the discrete sampling of rays, which can lead to undersampling errors in predicted impulse responses, especially in the interaction with diffracting edges [40]. To overcome this, ray tracing codes will generate a very large number of samples. For a structure as complex as the AFD office building, the computation times necessary to obtain a numerically accurate impulse response are prohibitively long. Another way to overcome undersampling is to use conical rays of energy instead of infinitely narrow rays, as described in the next section.

\subsubsection{Beamtracing}

Beam tracing is an acoustic modeling method that is fundamentally very similar to ray tracing. Beamtracing, however, radiates cone or pyramidshaped beams of energy from the source instead of the linear rays used in ray tracing. This method improves raytracing analysis in the ability to accommodate convex corners. In a beamtracing algorithm, if the beam is larger than the reflecting surface (as with a corner), the code can approximate the diffu-

sion effect by creating a new beam source [41] or by reallocating some of the sound energy into the diffuse field.

Beam tracing is a better suited method for this effort because it is more geometrically coherent than a ray tracing algorithm. The method described in [41] is especially useful because it is efficient in solving for maze structures. Their algorithm can solve for an entire building model with less than $20 \mathrm{MB}$ 
and in less than 5 minutes. It is suited for coarse 3D models without highly faceted surfaces, which easily encompasses the AFD office space. It is limited, however, as many beam tracing algorithms are: the geometric operations required to develop and utilize a beam tracing algorithm are generally complex [42], and it has yet to be incorporated into readily-accessible or commericial software. This would greatly slow the modeling procedure for the firefighter localization tests and make this method infeasible for the desired models.

\subsubsection{Finite Element Methods}

The Finite Element Method (FEM) is one of the most frequently used methods for solving the partial differential equations used to describe the behavior of physical systems. FEM uses a simple approximation of unknown variables to transform partial differential equations into algebraic equations that are easily solved using computers [43]. It involves four primary steps.

1. Creation of a physical model, describing the physical system in engineering terms, including geometry, physical laws and properties governing behavior, and boundary conditions.

2. Translation of the physical model into a mathematical model.

3. Construction of a numerical model that can be solved using a computer, where FEM is used to discretize the mathematical system.

4. Implementation of computer code to simulate behavior of physical system. 
FEM is generally limited to low frequencies and for simple environments due to large increases in computational time and storage space with frequency [41]. COMSOL Multiphysics ${ }^{T M}$ is a finite element solver. COMSOL has an Acoustics Module, which includes a Pressure Acoustics solver. To understand how COMSOL Multiphysics solves an acoustic problem, a brief review of acoustics is necessary.

The simplest form of the acoustic plane wave equation in the time domain is defined in Equation (3.1) [26],

$$
\frac{\partial^{2} p}{\partial t^{2}}=c^{2} \frac{\partial^{2} p}{\partial x^{2}}
$$

where $t$ is time, $x$ is a spatial coordinate, $c$ is the speed of sound in the medium, and $p$ is the acoustic pressure. The solution to this problem can be defined in either the time domain or the frequency domain. The time domain solution is generally given by the D'Alembert solution,

$$
p=f\left(x-c_{0} t\right)+g\left(x+c_{0} t\right) .
$$

By assuming that the solution is time-harmonic, however, the signal may be expanded into harmonic components via its Fourier series. The solution can be rewritten as shown in (3.3) and (3.4) and solved for in the frequency domain $(\omega)$ for individual frequencies using either sinusoids,

$$
p=p(x) \sin \omega t
$$


or complex exponentials,

$$
p=p(x) e^{i \omega t}
$$

By inserting this assumed solution into the time-domain full wave equation (Eq. (3.1)), the wave equation reduces to the Helmholtz equation:

$$
\nabla^{2} \hat{p}+k^{2} \hat{p}=0
$$

where $k$ is the acoustic wavenumber. COMSOL solves the Helmholtz equation at a given frequency across each element to give a full picture of the steady state sound field in an environment. This study, however, is not looking to find the steady state solution; it is looking to address the propagation of a finite pulse. COMSOL has a built-in transient solver, designed to solve the wave equation at each element. This solver is inefficient and can lead to numerical instabilities. To use COMSOL most efficiently, Fourier synthesis is used to identify an impulse response in the environment given the steady state solutions.

\subsubsection{Fourier Synthesis}

Fourier synthesis is used to convert an input signal to a full room response using the frequency domain solutions obtained analytically or numerically through COMSOL. Fourier synthesis compiles frequency domain finite element solutions and converts them to a full-spectrum time domain solution. 
Though the inefficiencies of finite element methods at high frequencies described above still apply, this was determined to be the best alternative for the desired acoustic mapping.

Some signal processing fundamentals are required to understand Fourier synthesis. The object of primary importance is the understanding of transfer functions and impulse responses. For any linear system, the relationship between the input and the output is given by the transfer function,

$$
P_{\text {out }}(\omega)=H(\omega) P_{\text {in }}(\omega)
$$

where $P_{\text {out }}(\omega)$ is the output in the frequency domain, $P_{\text {in }}(\omega)$ is the input in the frequency domain, and $H(\omega)$ is known as the transfer function. Through the Fourier transform (Eq. (3.7) and (3.8)), each of these components can be changed between the frequency domain and the time domain, as shown in (3.9). A multiplication in the frequency domain, as shown in (3.6), correlates to a convolution integral in the time domain, as shown in Equation (3.9):

$$
\begin{gathered}
\hat{p}(x, \omega)=\int_{-\infty}^{+\infty} p(x, t) e^{-i \omega t} d t, \\
p(x, t)=\int_{-\infty}^{+\infty} \hat{p}(x, \omega) e^{i \omega t} d t, \\
p_{\text {out }}(t)=h(t) * p_{\text {in }}(t),
\end{gathered}
$$


where $p_{\text {out }}(t), p_{\text {in }}(t)$, and $h(t)$ are the time-domain counterparts to the variables in Equation (3.6). This is true for any linear system. The central idea behind Fourier synthesis is that one can very efficiently solve the Helmholtz equation, either analytically or through a tool such as COMSOL, for a spectrum of frequencies that are then compiled into the transfer function, $H(\omega)$. With a known set of signal processing parameters, this can be Fourier transformed into the time domain to obtain an impulse response or multiplied by the input signal spectrum to obtain the output signal spectrum. The output signal spectrum can be transformed back into the frequency domain, providing the time-domain response to the specified input signal.

To demonstrate the process and prove that it works both analytically and using COMSOL, an example is provided.

Given a perfectly square room, what is the response of a unit impulse input at the center?

1. Define signal processing components.

First, some signal processing components must be defined. These will be important in the Fourier transform process. For Fourier synthesis, they can be user-defined. It is generally necessary to define two of the components, and the rest will be dependent on the two independent components.

For example, defining an input frequency of interest will guarantee that the Nyquist frequency is well above the frequency range of interest. 
Defining a sampling rate will define an input frequency of interest, above which you may near the Nyquist frequency of your transfer function. In this example, $F_{s}$ is specified to be $8000 \mathrm{~Hz}$. This gives a time resolution of $0.000125 \mathrm{~s}$, which in turn gives a grid resolution of $4.3 \mathrm{~cm}$. For a grid resolution of $8 \mathrm{x}$ the wavelength of interest, this gives a wavelength of interest of $34.3 \mathrm{~cm}$. This wavelength correlates to an input frequency of $1000 \mathrm{~Hz}$, which is an effective cap on a study frequency.

Table 3.1: Necessary Signal Processing Values

\begin{tabular}{cccc}
\hline \hline Constant & Description & Equation & Value \\
\hline$F_{0}$ & Input Frequency of Interest & User-Defined & $1000 \mathrm{~Hz}$ \\
$c_{0}$ & Sound Speed & User-Defined & $343 \mathrm{~m} / \mathrm{s}$ \\
$\lambda$ & Wavelength of Interest & $\lambda=c_{0} / F_{0}$ & $34.3 \mathrm{~cm}$ \\
$\mathrm{dx}$ & Grid Resolution & $d x=\lambda / 8$ & $4.3 \mathrm{~cm}$ \\
$\mathrm{dt}$ & Incremental Time & $d t=d x / c_{0}$ & $0.000125 \mathrm{~s}$ \\
$T$ & Period & $T=10 / F_{0}$ & $0.01 \mathrm{~s}$ \\
$F_{s}$ & Sampling Frequency & $F_{s}=1 / d t$ & $8000 \mathrm{~Hz}$ \\
$d f$ & Incremental Frequency & $d f=1 / T$ & $100 \mathrm{~Hz}$ \\
$\mathrm{~N}$ & Number of Samples & $N=F_{s} T$ & 80 \\
\hline
\end{tabular}

2. Calculate transfer function at each point across a user-defined grid $[\mathrm{X}, \mathrm{Y}]$. For a perfectly square room with rigid walls, the analytical solution to the Helmholtz equation is given in (3.10).

$$
H(\omega)=\sum_{m, n}^{\infty}\left[A \cos \frac{m \pi x}{L} \cos \frac{n \pi y}{L}+\frac{p_{0}}{\omega^{2}}\right]
$$

The user should define a uniformly spaced grid across the space $[\mathrm{X}, \mathrm{Y}]$, 


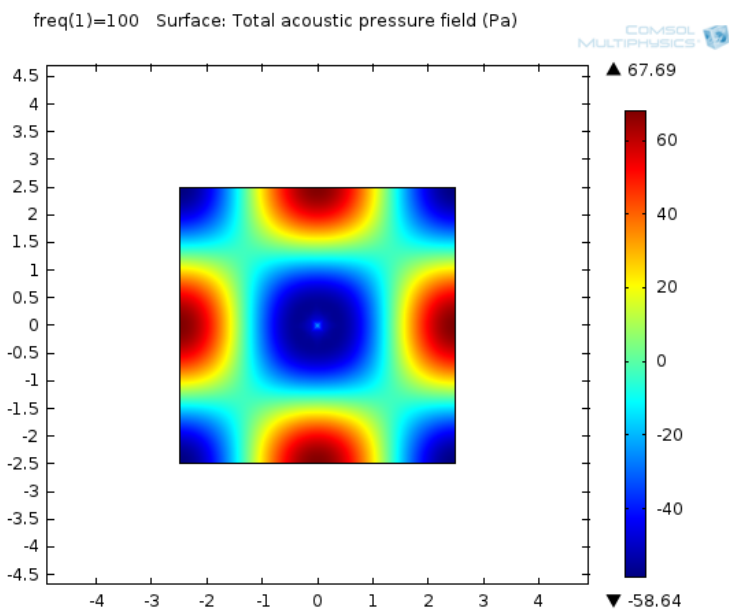

Figure 3.2: COMSOL solution of square room example at $100 \mathrm{~Hz}$. The $\mathrm{x}$-axis marks the width of the room and the $y$-axis marks the height of the room, each equal to 5 meters. The pressure gradient is given in decibels, scaled relative to a finite amplitude pulse in a room with lossless boundaries.

where $-\frac{L}{2}<X<\frac{L}{2}$ and $-\frac{L}{2}<Y<\frac{L}{2}$. Then, (3.10) should be solved at each point $(\mathrm{X}, \mathrm{Y})$ of that grid and assembled into a $\mathrm{X} \times \mathrm{Y}$ matrix.

The same process can be performed in COMSOL by drawing the geometry and creating a study to export the solution at each of the same $[\mathrm{X}, \mathrm{Y}]$ data points, as shown in Figure 3.2, which will be necessary for geometries that cannot be solved analytically.

3. Repeat transfer function calculation across frequency vector [0:df:Fs/2]. The incremental frequency and sampling rate are defined in the first item on the list. The incremental frequency is dependent on the period of the input signal, which can be specified as a number much greater than the input frequency wavelength. The solution is only iterated through the 
Nyquist frequency because the Fourier transform is conjugate symmetric (Equation (3.11)). Because Fourier synthesis numerically implements the Fourier transform using the fft function in Matlab, the second half of the $\mathrm{fft}$ is constructed by calculating and inserting the complex conjugate of the originally calculated spectrum:

$$
\hat{p}(-\omega)=\hat{p}^{*}(\omega) .
$$

This results in a three-dimensional matrix representing the 2D spatial sample and frequency vector

4. Perform convolution with input signal.

Each point in space now has a full spectrum response, from 0 to $\mathrm{Fs} / 2$. As described previously, this response can either be transformed to give a time-domain impulse response at that point or multiplied by the input signal spectrum and transformed to show the time response to an input signal. Plotting the time response to a unit impulse at each point demonstrates a visual representation of how the sound pressure changes with time. Taking the RMS average of this response gives the modal structure of the space, as shown in Figure 3.3. Using another signal of interest for the input signal, such as a pure sine tone, will show the modal structure of that particular frequency in the space, like the 100 $\mathrm{Hz}$ modal response shown in Figure 3.2. 


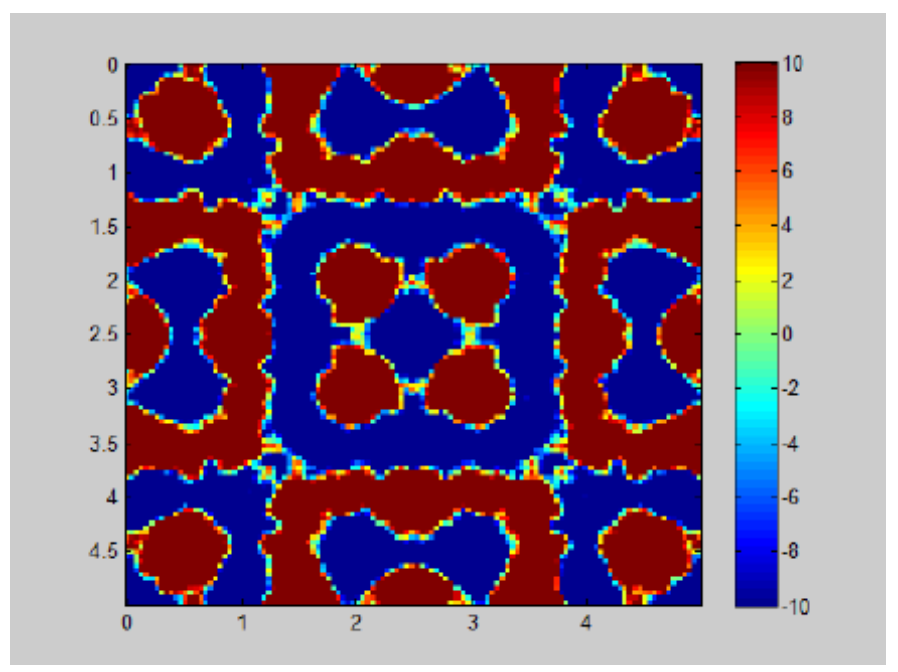

Figure 3.3: Time-averaged modal response of square room example. The pressure peaks (red) and nulls (blue) represent the $2 D$ modal structure of the space. The $\mathrm{x}$-axis marks the width of the room and the $y$-axis marks the height of the room, each equal to 5 meters. The pressure gradient is given in decibels, scaled relative to a finite amplitude pulse in a room with lossless boundaries.

\subsection{Sound Field for PASS Alarm}

Using the method described above, the modal structure of the sound field in the AFD warehouse was calculated and is shown in Figure 3.4.

The input response to a unit impulse gives the most basic modal structure of the space. There were several assumptions made in the creation of the model. First, the model assumed perfectly rigid walls. This is most easily observed in the closet in the bottom-center of the warehouse. Because the door to this room was closed in the test, it is perfectly sealed in the COMSOL model. No sound transmission occurs through the walls, which is not a perfect recreation of the test. As for the rest of the walls, gypsum is a highly reflective 


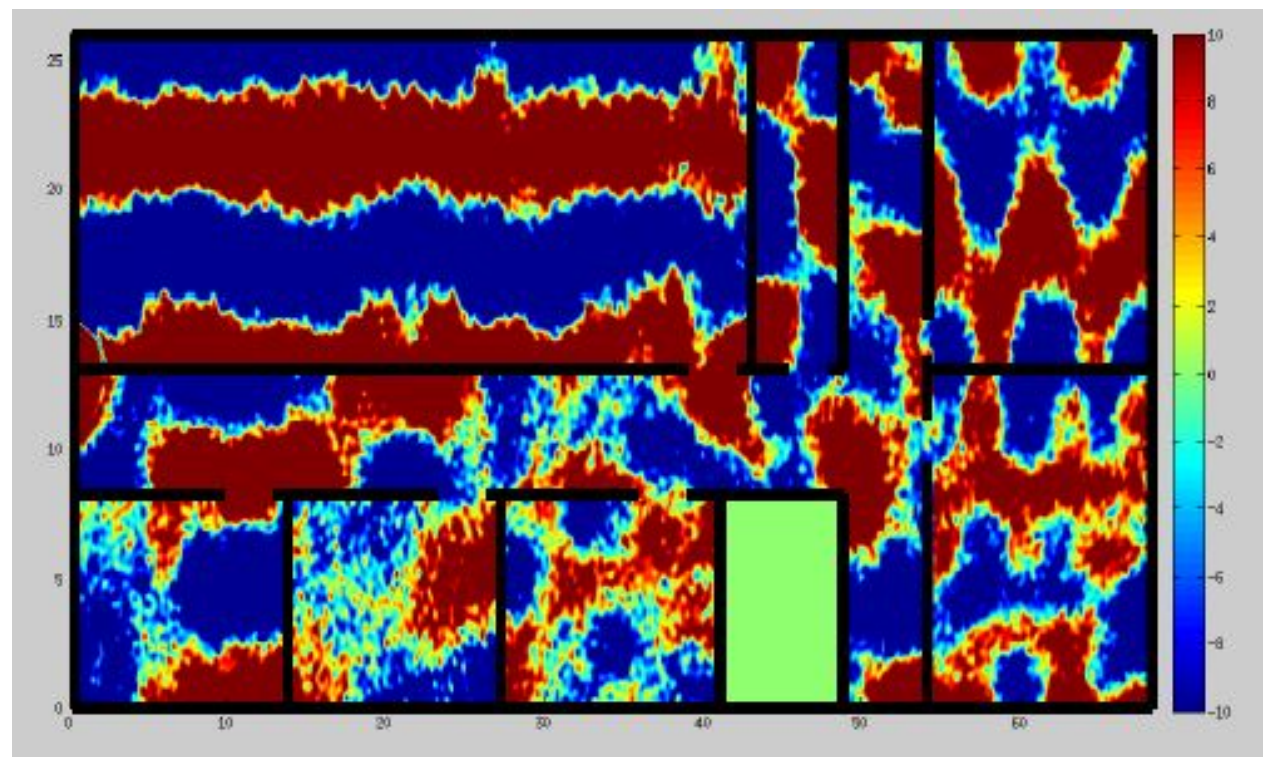

Figure 3.4: Time-averaged modal response to finite amplitude impulse at PASS location, layered on top of the floorplan shown in Figure 2.1. The pressure gradient is given in decibels, scaled relative to the finite amplitude pulse in a room with lossless boundaries. 
material and more work to determine whether further computational accuracy would be useful is described in the following sections. The only losses from this system come from losses in air, which are generally minimal. One alternative to adding losses into the model would be to estimate overall level decreases empirically and scale the overall modal structure accordingly.

Another assumption was that the PASS speaker acted as a point source. For very low frequencies, this is acceptable, but the directionality of the speaker might cause problems at the mid-range frequencies.

\subsection{Sound Fields for Noise Sources}

This procedure will be repeated for all four noise sources separately in an effort to eliminate coherent addition or incoherent subtraction of sources. The noise sources are squared and summed, and the square root of the final sum provides the overall noise field.

Special attention must be paid to the scaling of all sources. All transfer functions were calculated with the same pressure input, so there is consistency across all calculations. However, because the system is largely lossless, the general scaling of each and final $\mathrm{dB}$ value given is too high to be assumed accurate. The noise field are scaled based on the input levels of each individual devices as measured in previous work (please refer to Section 1.3.1). 


\subsection{Field where PASS is in Audible Range}

There are many potential ways to map where the PASS might be audible. One option would be to directly model the SNR based on the unit impulse functions, where black represents a positive SNR and white represents a negative SNR.

More detailed models can be created. Some further options to consider would be the implementation of frequency-dependent masking effects. The losses caused by the firefighter PPE, as described in Section 1.3.3, could be incorporated as a system-wide $5 \mathrm{~dB}$ decrease in SNR. Finally, the most difficult (and probably most important) variation would be to include temporal effects of the different signals. No signal presents a constant decibel level. Each signal has peaks and nulls throughout, and a major point of identification is waiting until nulls in noise signals coincide, so the potential for identifying the PASS signal is optimized. One way to model this might be to sum the low levels of the noise signals and the peak signal for the PASS signal to compare to a general plot where all signals are maximized. This should be determined on a case-by-case basis for future iterations of this modeling effort.

\subsection{Potential for Auralization}

One final goal of this modeling effort was to be able to auralize sounds, as an easily-understandable example for firefighters and nontechnical audiences. Auralization is the technique of creating audible sound files from numerical (simulated, measured, or synthesized) data [44]. The principle of au- 
ralization follows the signal processing methodology described earlier in this section, where the input signal would be an audio recording of a PASS device in an anechoic setting.

Unfortunately, the finite element basis of this analysis limits the available frequency range of the simulated transfer function. While the PASS device contains frequencies up to $4000 \mathrm{~Hz}$, it contains harmonics much higher than that. Additionally, an audio recording of PASS or noise sources would be recorded at an audio sampling rate of $44.1 \mathrm{kHz}$. For convolution, the signal and transfer function must generally have equal sampling rates, and $44.1 \mathrm{kHz}$ (with a simulation up to the Nyquist frequency of approximately $22 \mathrm{kHz}$ ) would be impractically large to achieve using this method. However, the potential for decimation of the audio files exists to achieving the necessary sampling rate for convolution.

Decimation is the digital process used to reduce the sampling rate of a signal [45]. It is the inverse process of interpolation, where only every integer multiple sample is retained in the processed signal. Special steps must be taken to prevent aliasing in the downsampled signal, known as an anti-aliasing filter. A decimating filter is shown in Equation (3.12),

$$
y(n)=\sum_{k=0}^{K-1} x(n M-k) \cdot h(k),
$$

where $h$ is the transfer function, $K$ is the number of samples in the transfer function, $x$ is the signal being downsampled, and $M$ is the integer multiple of 
interest. Once the signal has been decimated to the sampling rate of interest, it can be convolved with the transfer function. Then the resulting signal can be interpolated back to the audio sampling rate using the inverse of the filter described above and auralized.

\subsection{Conclusion}

Several methods of modeling a sound field are described in this chapter, including ray tracing, beam tracing, and finite element modeling. The finite element method proved to be the most efficient modeling method for the firefighter localization tests. Fourier synthesis is used to convert the steadystate frequency response into a full-spectrum transfer function at each point in the space. Once the transfer function is calculated, it can be used to show modal structures or compare signal levels to noise levels at locations of interest throughout the space. Further work is required to fully implement an audibility map, but the basis for the continuation of the concept is provided here. 


\section{Chapter 4}

\section{Improved Boundary Conditions}

All modeling efforts to this point have assumed perfectly rigid boundaries. For the 2D model, these boundaries entirely consist of gypsum wallboard, which is very commonly used for wall construction in the United States. This research team decided to better quantify the acoustic effect of fire on gypsum wallboard to see if these assumptions are valid or if more computational emphasis should be placed on boundary conditions dependent on the state of fire.

\subsection{Background of Gypsum Wallboard Chemistry}

Gypsum rock (primarily calcium sulphate dihydrate) is mined from quarries and crushed to aggregate size. Roughly $75 \%$ of the bound water is eliminated by heating the crushed rock in a kiln to about $175^{\circ} \mathrm{C}$. This process is referred to as calcination and the final product is plaster of paris (formally known as gypsum plaster). When the correct amount of water is added back to the calcinated gypsum, the liquid mixture is poured onto the lower sheet

of paper and the upper sheet of paper is applied to the mix. The board is passed through rollers as the plaster sets, forming what we recognize as 
gypsum wallboard. The paper is chemically and mechanically bonded to the core. The board is kiln-dried to remove all excess moisture.

\subsection{Fire Model}

The chemical composition of gypsum wallboard is $\mathrm{CaSO}_{4} \cdot 2 \mathrm{H}_{2} \mathrm{O}$, or calcium sulphate dihydrate. It contains about $21 \%$ (by mass) chemically combined water. At elevated temperatures (such as those found in fire conditions), the water will disassociate in a process known as dehydration. Full dehydration occurs when both water molecules have evaporated. This dehydration occurs in two main steps of endothermic decomposition. This first step of dehydration is shown in Equation (4.1):

$$
\mathrm{CaSO}_{4} \cdot 2 \mathrm{H}_{2} \mathrm{O}+\text { heat } \longrightarrow \mathrm{CaSO}_{4} \cdot 0.5 \mathrm{H}_{2} \mathrm{O}+1.5 \mathrm{H}_{2} \mathrm{O} \text {. }
$$

The second step occurs when the rest of the water disassociates, as shown in Equation (4.2):

$$
\mathrm{CaSO}_{4} \cdot 0.5 \mathrm{H}_{2} \mathrm{O}+\text { heat } \longrightarrow \mathrm{CaSO}_{4}+0.5 \mathrm{H}_{2} \mathrm{O} \text {. }
$$

$\mathrm{Yu}$ [46] examined dehydrating gypsum to better quantify the thermal properties related to the dehydration reaction, such as dehydration temperatures and required energy. $\mathrm{Yu}$ showed experimentally that the heating rate does not affect the dehydration temperature, but it influences the ending tem-

perature, indicating that the energy needed for dehydration is dependent on 
both the temperature and the heating time. He proved experimentally that initial dehydration reaction takes place from $80^{\circ} \mathrm{C}$ to $120^{\circ} \mathrm{C}$. An additional minimal mass loss occurs between $120^{\circ} \mathrm{C}$ and $220^{\circ} \mathrm{C}$, finalizing the disassociation of the water. His findings indicate that given sufficient heating time, the first step of dehydration can complete at $80^{\circ} \mathrm{C}$ and the second step can reach completion at $120^{\circ} \mathrm{C}$.

Others have attempted to try to quantify these reaction temperatures. Nguong [47] performed extensive calcination depth calculations in the development and verification of his probe method. Thermocouples were placed at $4 \mathrm{~mm}, 8 \mathrm{~mm}, 12 \mathrm{~mm}$, and $16 \mathrm{~mm}$ under the surface of a gypsum board sample that was heated from one side at a constant heat flux. The temperatures plateau at about $100^{\circ} \mathrm{C}$, which is where Nguong estimates the temperature at which the dehydration process occurs. The temperature stayed at this temperature until the dehydration reaction was completed.

This report first seeks to create a numerical model of density loss and dehydration depth across a section of gypsum wallboard. The first step was to use a finite difference approximation to create a discretized energy balance at discrete nodal points across the wall (neglecting any dehydration effects, i.e. for heat transfer below $80^{\circ} \mathrm{C}$ ). For the interior nodes, this energy balance is shown in Equation (4.3),

$$
\rho c \frac{\partial T}{\partial t}=k \frac{\partial^{2} T}{\partial x^{2}}
$$


where $\rho$ is the density, $c$ is the thermal capacity, and $k$ is the thermal conductivity. Introducing $p$ to represent an incremental time step and $m$ to represent the spatial step, the discretized heat equation is shown in Equation (4.4),,

$$
\frac{1}{\alpha} \frac{T_{m}^{p+1}-T_{m}^{p}}{\Delta t}=\frac{T_{m+1}^{p}+T_{m-1}^{p}-2 T_{m}^{p}}{(\Delta x)^{2}},
$$

where $\alpha$ is the thermal diffusivity. $F_{0}$ is defined as the discretized Fourier number and $B i$ is defined as the discretized Biot number, as shown in Equation (4.5) and Equation (4.6):

$$
\begin{gathered}
F_{0}=\frac{\alpha \Delta t}{(\Delta x)^{2}}, \\
B i=\frac{h \Delta x}{k} .
\end{gathered}
$$

The discretized Fourier number and Biot number are important in determining an acceptable timestep for a given spatial discretization in the explicit method (to ensure stability), as discussed in detail in Incropera and DeWitt [48]. The discretized heat equation is shown in Equation (4.7):

$$
T_{m}^{p+1}=\frac{\alpha \Delta t}{(\Delta x)^{2}}\left(T_{m+1}^{p}+T_{m-1}^{p}\right)+\left(1-\frac{2 \alpha \Delta t}{(\Delta x)^{2}}\right) T_{m}^{p} .
$$

For the exterior nodes, a convective heat transfer term is included, so the entire discretized heat equation becomes:

$$
h A\left(T_{\infty}-T_{n}^{p}\right)+\frac{k A}{\Delta x}\left(T_{n+1}^{p}-T_{n}^{p}\right)=\rho c A \frac{\Delta x}{2} \frac{T_{n}^{p+1}-T_{n}^{p}}{\Delta t} .
$$


These equations will be valid until a node reaches one of the two dehydration temperatures $\left(T_{d 1}=80^{\circ} \mathrm{C}\right.$ and $\left.T_{d 2}=120^{\circ} \mathrm{C}\right)$ [46]. When a node reaches a dehydration temperature, the $\frac{\partial T}{\partial t}$ term in the energy balance will go to zero and be replaced by an energy sink, $\dot{m}_{\mathrm{H} 2 \mathrm{O}}^{\prime \prime} L_{v}$, representing the evaporation rate as the water is chemically released at the dehydration interface. The new energy balance for the interior nodes becomes:

$$
\begin{gathered}
\dot{m}_{\mathrm{H} 2 \mathrm{O}}^{\prime \prime \prime} L_{v}=k \frac{\partial^{2} T}{\partial x^{2}}, \\
\dot{m}_{\mathrm{H} 2 \mathrm{O}}^{\prime \prime \prime}=\frac{k}{L_{v}} \frac{\partial^{2} T}{\partial x^{2}}=\frac{k}{L_{v}} \frac{T_{m+1}^{p}+T_{m-1}^{p}-2 T_{m}^{p}}{(\Delta x)^{2}} .
\end{gathered}
$$

The new energy balance for the surface nodes becomes:

$$
\begin{aligned}
& h\left(T_{\infty}-T_{n}^{p}\right)+\frac{k}{\Delta x}\left(T_{n+1}^{p}-T_{n}^{p}\right)=\frac{\dot{m}_{H 2 O}^{\prime \prime} L_{v}}{\Delta x}, \\
& \dot{m}_{H 2 O}^{\prime \prime \prime}=\frac{h \Delta x}{L_{v}}\left(T_{\infty}-T_{n}^{p}\right)+\frac{k}{L_{v}}\left(T_{n}^{p}-T_{n+1}^{p}\right) .
\end{aligned}
$$

This gives a model for the total mass loss, as shown in Equation (4.13):

$$
\dot{m}_{H 2 O}^{\prime \prime \prime}(t+\Delta t)=\dot{m}_{H 2 O}^{\prime \prime \prime}(t)-\dot{m}_{H 2 O}^{\prime \prime \prime} \Delta t .
$$

The latent heat of vaporization of water at atmospheric pressure was used $(2257 \mathrm{~kJ} / \mathrm{kg})$. In the first step of the dehydration process, 1.5 moles of 
$\mathrm{H}_{2} \mathrm{O}(27 \mathrm{~g})$ disassociates from one mole of $\mathrm{CaSO}_{4} \cdot 2 \mathrm{H}_{2} \mathrm{O}(172 \mathrm{~g})$. This represents a $16 \%$ mass loss. In the second step of the dehydration process, 0.5 moles of $\mathrm{H}_{2} \mathrm{O}(9 \mathrm{~g})$ disassociate from a mole of $\mathrm{CaSO}_{4} \cdot 0.5 \mathrm{H}_{2} \mathrm{O}(145 \mathrm{~g})$, representing a $6 \%$ mass loss. When the mass loss equals $16 \%$ and $6 \%$, respectively, and the dehydration process has completed, the energy input will go back to increasing the temperature.

\subsection{Acoustic Properties}

One would not traditionally measure the absorptivity of gypsum wallboard in an impedance tube; highly reflective materials cause problems because they cause standing waves to occur in the tube. Some tubes can be designed to combat this by applying a porous absorber coating or lining inside the impedance tube near the loudspeaker, but that is not possible with the construction of the ETS-Lindgren impedance tube used in this study.

Typically, gypsum wallboard is measured using reverberation time methods and is very rarely measured singularly. It is typically measured in an assembly, and sound transmission class (rather than acoustic impedance or absorption coefficient) is the provided metric for design.

Lossi [49] reports that the density of gypsum wallboard is $960 \frac{\mathrm{kg}}{\mathrm{m}^{3}}$ and the sound speed is $6800 \mathrm{~m} / \mathrm{s}$. This leads to an acoustic impedance of $6.52 \times 10^{6}$ Rayls, which is closely comparable to brick $\left(6.66 \times 10^{6}\right.$ Rayls $)$.

The primary difference between gypsum and brick regarding sound 
transmission, however, is density. Brick is much more dense than gypsum

wallboard $\left(1800 \frac{\mathrm{kg}}{\mathrm{m}^{3}}\right)$, and the additional mass prevents much sound transmission according to the acoustic mass law.

\subsubsection{Impedance Tube Methods}

An impedance tube is an acoustical instrument used to measure the specific acoustic impedance of materials [27]. Once the specific acoustic impedance of a material is determined, the absorption and reflection coefficients can be calculated using methods described below. This information can be used to increase the accuracy of the acoustic models.

The impedance tube itself consists of a long tube with a sound source at one end and a sample of the material of interest, in this case gypsum wallboard, at the other. Plane waves are generated using a broadband signal from the sound source, and the sound pressure is measured simultaneously at two locations in the tube's wall. The transfer function between the two microphones is measured.

Once the transfer function has been obtained, the complex reflection coefficient can be calculated using Equation (4.14):

$$
R=|R| e^{j \phi R}=\frac{H-e^{-j k s}}{e^{j k s}-H} e^{j 2 k(l+s)} .
$$

where $H$ is the transfer function of the two microphone signals (corrected for microphone mismatch), $s$ is the center-to-center spacing between the micro- 


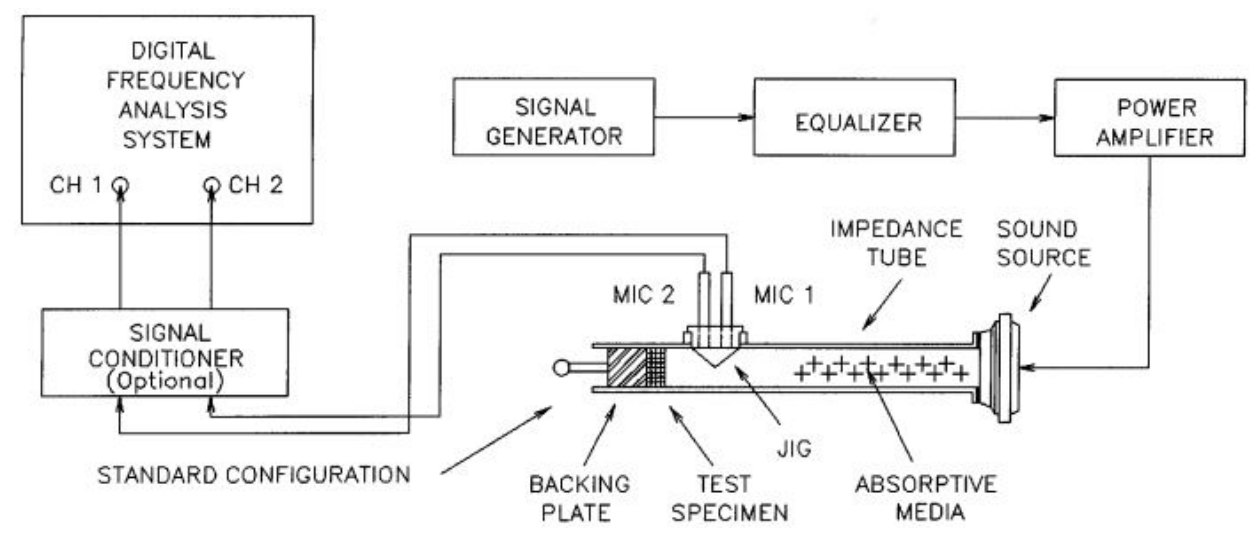

Figure 4.1: Schematic of impedance tube analysis. Taken from ASTM E1050-98.

phones $(\mathrm{m}), k$ is the acoustic wave number, and $l$ is the distance from the test sample to the center of the nearest microphone $(\mathrm{m})$.

Once this complex reflection coefficient is calculated, acoustic relationships can be used to find many other metrics, including the normal incidence sound absorption coefficient, $\alpha$, and the normal specific acoustic impedance ratio, $z / \rho c$.

\subsubsection{ETS-Lindgren Impedance Tube}

ETS-Lindgren, a company specializing in third-party acoustic testing, has an impedance tube that was used in this experiment.

The tube is made of a rolled, square aluminum tube. The tube is approximately 1.93 meters long. The spacing between the microphones is approximately $0.0286 \mathrm{~m}$.

The tube was less than ideal for measuring gypsum wallboard because 


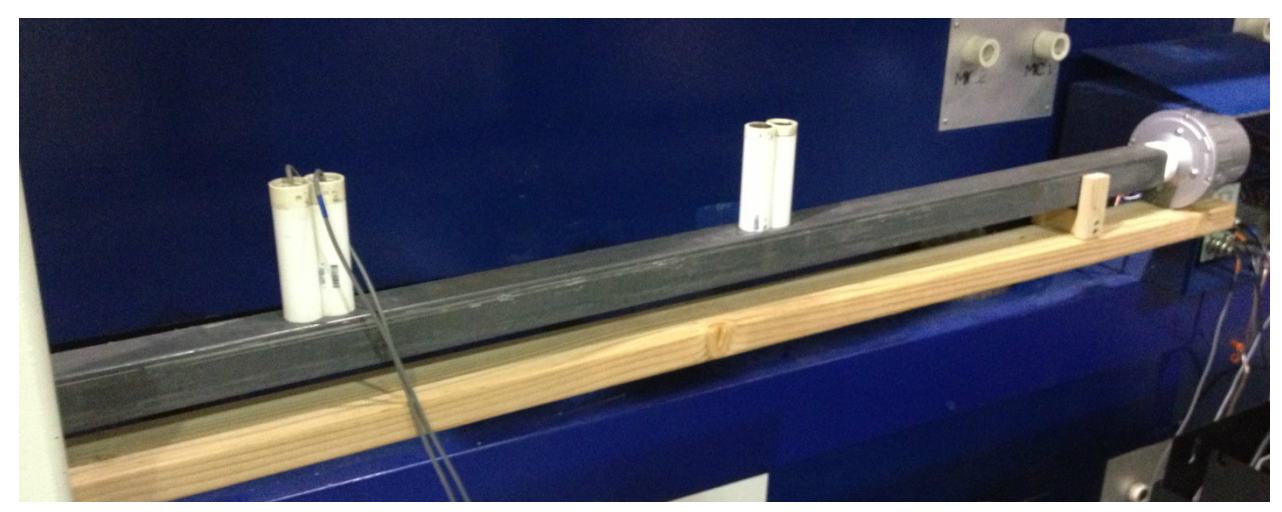

(a) High-Frequency Impedance Tube

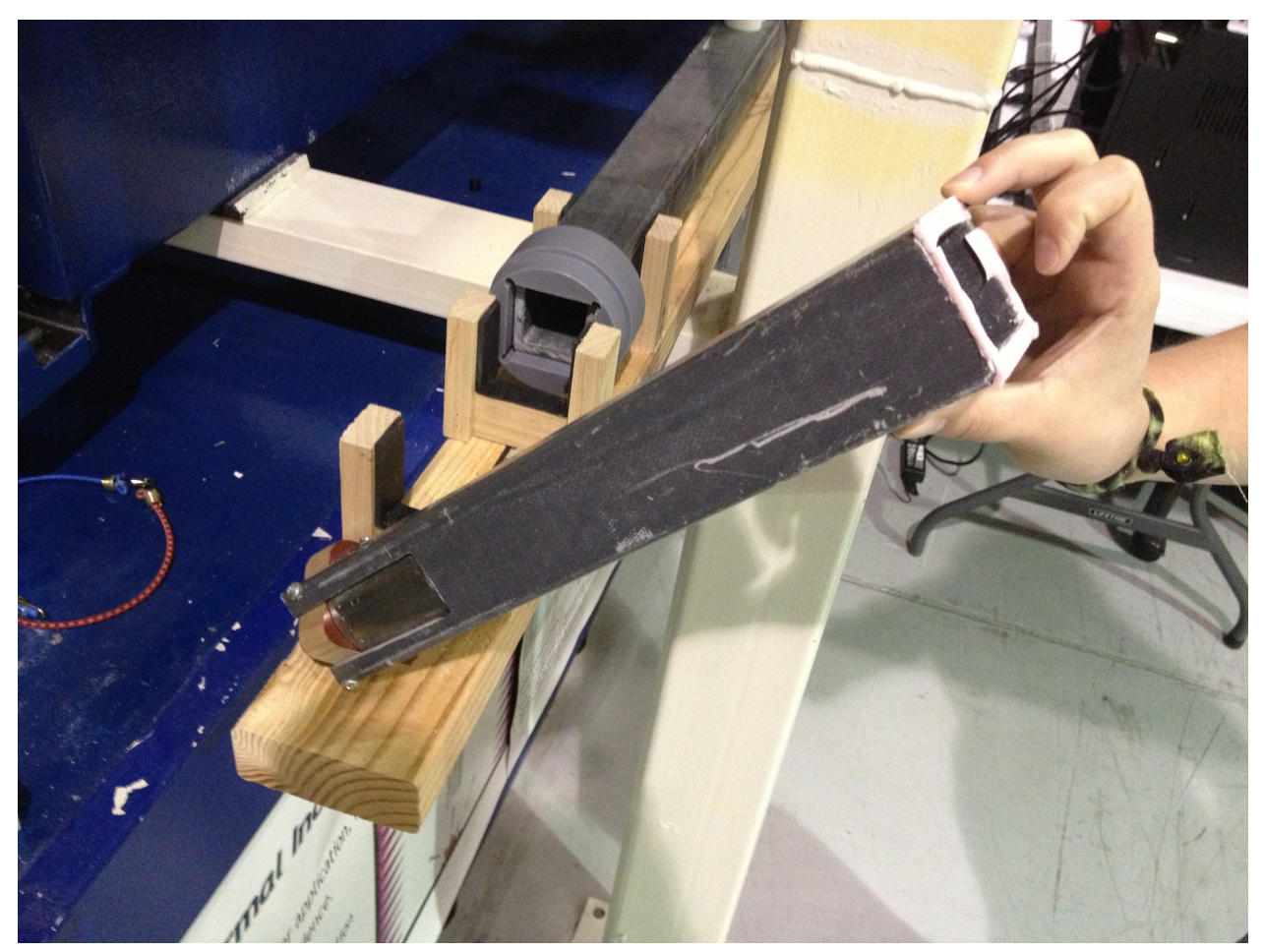

(b) Impedance Tube Backing Plate

Figure 4.2: The ETS-Lindgren High-Frequency Impedance Tube. 
the backing plate for the test specimen was approximately $0.2413 \mathrm{~m}$ (9.5 in) deep. The impedance tube was originally designed to measure the absorptivity of acoustic foam, which comes in thick wedges and is pliable enough to squeeze into such a space. Gypsum wallboard, on the other hand, is only $1.65 \mathrm{~cm}(0.65$ in) thick and very rigid, making it infeasible to place against the backing plate of the tube.

The options were to place the gypsum at the edge of the tube and seal with vacuum grease, accounting for the air gap behind the gypsum in the calculations, or to make an alternate backing plate to better accommodate the sample. Both options were explored.

Additionally, there is no absorptive material in the tube near the speaker. Because the tube is so long and narrow, there were problems with standing waves in the tube that were not easily addressed.

\subsubsection{Impedance Tube Procedure}

The procedure for taking measurements in the ETS-Lindgren impedance tube was as follows:

1. Calibrate the microphones into the Data Physics software (used for data acquisition in these experiments)

2. Place mics in original configuration, place anechoic foam in the sample holder, and measure transfer function and coherence 
3. Switch the microphone configuration and measure the transfer function and coherence of anechoic foam sample

4. Switch microphones back to original configuration

5. Place gypsum in sample holder

6. Measure transfer function and coherence and analyze according to ASTM E1050-98.

\subsection{Measurements}

\subsubsection{Impedance Tube Benchmarking Tests}

First, two test scenarios were used to test the fidelity of the impedance tube. The first was to measure the absorption coefficient of the anechoic foam (see Figure 4.3(a)), which one would expect to be very close to 1 . These tests used a pink noise generator to provide the input signal, as specified in ASTM E1050.

The coherence is also shown in Figure 4.3(b). The coherence is a metric that measures the correlation between two signals; it is often used to detect the presence of noise in digital signal processing. It is useful in impedance tube testing because it can identify the presence of disruptive standing waves (peaks and nulls within the tube).

The results are encouraging. There is a small dip in coherence above $4500 \mathrm{~Hz}$, but that is to be expected. Per ASTM E1050-98, the upper frequency limit of the tube is to be defined as 


$$
f_{u}<\frac{K c}{d}
$$

where $f_{u}$ is the upper frequency limit $(\mathrm{Hz}), c$ is the speed of sound in the tube $(\mathrm{m} / \mathrm{s}), d$ is the diameter (or largest section dimension) of the tube $(\mathrm{m})$, and $K$ is a constant, 0.5 (for rectangular tubes). Our tube is $0.038 \mathrm{~m}$ square, and the speed of sound in the tube is $343 \mathrm{~m} / \mathrm{s}$. This puts the upper limit of the ETS-Lindgren tube at $4513 \mathrm{~Hz}$. Above this frequency, cross-modes have started to affect the coherence between the two microphones.

Another test was performed with the sample holder with no sample inside, which acoustically mimics a finite-length, one-dimensional waveguide with a rigid termination. The measured specific acoustic impedance can be compared to the expected specific acoustic impedance of a rigid stop.

The solid line in Figure 4.4 represents the theoretical acoustic impedance for a rigid stop in a tube of this size, defined by

$$
Z_{t}=-\frac{1}{\rho c} \cot (k d)
$$

where $k$ is the wavenumber and $d$ is the distance from the rigid stop to the measurement point - in this case, the edge of the sample holder.

The data points in Figure 4.4 represent the measured specific acoustic impedance in the tube. There is good agreement between the two, although it begins to deviate above $4500 \mathrm{~Hz}$, as expected. 
The coherence plot (Figure 4.4(b)) is not clean. The first assumption was that this was caused by standing wave structures in the tube. If there is a null at or near one of the microphone locations, coherence drops significantly. This is theoretically demonstrated in the coherence data, as towards higher frequencies, you get double dips (presumably due to the presence of more null locations) versus lower frequencies (fewer null locations).

There are two lengths of interest: from rigid end to the first microphone $(0.7366 \mathrm{~m})$ and from rigid end to the second microphone $(0.7652 \mathrm{~m})$. The coherence nulls should occur at frequencies where the length is equal to quarter wavelength multiples, as shown in (4.17):

$$
f_{n}=\frac{(2 n-1) c}{2 L}
$$

Where $c$ is the speed of sound, $L$ is one of the end-to-microphone distances described above, and $n$ is an integer multiple (i.e. $n=1,2,3 \ldots$. These frequencies are compared to the nulls in Table 4.1.There is good agreement. The only way to decrease these resonances would be to add absorptive material near the speaker, which for reasons described previously was infeasible with this impedance tube.

\subsubsection{Gypsum Measurements}

After the impedance tube was tested by itself, a piece of gypsum was placed in the sample holder and measured. 
Table 4.1: Resonance Frequencies of Tube

\begin{tabular}{cccc}
\hline \hline $\mathrm{n}$ & End-to-Mic1 (Hz) & End-to-Mic2 (Hz) & Coherence Nulls (Hz) \\
\hline 1 & 116.4 & 112.5 & - \\
2 & 349.2 & $\mathbf{3 3 6 . 2}$ & $\mathbf{3 3 7}$ \\
3 & $\mathbf{5 8 2 . 1}$ & 560.3 & $\mathbf{5 8 7}$ \\
4 & 814.9 & 784.4 & - \\
5 & $\mathbf{1 0 4 7 . 7}$ & 1008.6 & $\mathbf{1 0 5 0}$ \\
5 & 1280.5 & $\mathbf{1 2 3 2 . 7}$ & $\mathbf{1 2 3 8}$ \\
\hline
\end{tabular}

The absorption coefficient of the gypsum wallboard sample is presented in Figure 4.5. The data are not what should be expected-in comparison, the blue line represents the octave-band absorption data presented in most standard specifications [50]. The measured absorption presents more losses than one would expect from gypsum wallboard.

The next iteration used a new sample holder with less space between the sample and the rigid backing plate $(4.365 \mathrm{~cm})$. It still did not mount the gypsum wallboard flush against the rigid back, but the distance between the two decreased. It was not possible to create a stop short enough to place the gypsum flush against the rigid backing due to the geometry of the tube's mounting.

The coherence improved, implying that something about the sample holder's structure is causing the resonances in the first place. However, for all measurements taken with the new sample holder, the absorption coefficient shows a large spike around $3000 \mathrm{~Hz}$ (up to an absorption coefficient of 0.7 - for reference, this is approximately equal to the absorption of acoustic drapery). 
This correlates with an improvement in coherence.

\subsubsection{Burned Gypsum Measurements}

Although the data obtained for unburned gypsum was not within a range of expected values, a burned sample was measured for the purposes of a general comparison.

As mentioned in the gypsum chemistry section, $16 \%$ mass loss represents complete dehydration of a sample. However, gypsum will rehydrate by about $4 \%$ between time in the oven and a longer period of time after heat exposure. Because no oven was available at the impedance tube facility, rehydration was inevitable (but measurable).

The initial mass of the gypsum sample was $72.5 \mathrm{~g}$. It was placed in a lab convection oven for 60 minutes at $200^{\circ} \mathrm{C}-120^{\circ} \mathrm{C}$. After heat exposure, the gypsum sample measured $60.2 \mathrm{~g}$, approximately a $13 \%$ mass loss.

In a fire, the ablation process would proceed in a similar manner. One difference between the two is that the paper layer would probably ignite and burn away. However, this limits the structural integrity of the sample, and when this was attempted, the sample could not stand alone in the sample holder. The pressure to put it in place caused the dehydrated gypsum to crumble.

The burned sample used the same setup as the unburned sample presented in the section above. The shorter sample holder was used. 
Figure 4.8 compares the measured absorption coefficients of the burned and unburned gypsum samples. The discrepancy is difficult to discern visually, but there is less than a $0.5 \%$ deviation between the two measurements. Regardless of the unknown losses causing the absorption to be so high, there is negligible difference between an unburned sample and a fully deydrated sample.

\subsection{Conclusion}

Although there were unresolved losses in the impedance tube, a sideby-side comparison of an intact gypsum sample and a fully dehydrated gypsum sample shows that the change in specific acoustic impedance and absorption coefficient is negligible. Structural failure would occur well before the occurrence an acoustic change worthy of modeling effort. Future work with the ETS-Lindgren impedance tube should implement improvements in the construction of the tube to eliminate the unknown losses, but for the purposes of this report, the results are sufficient. 


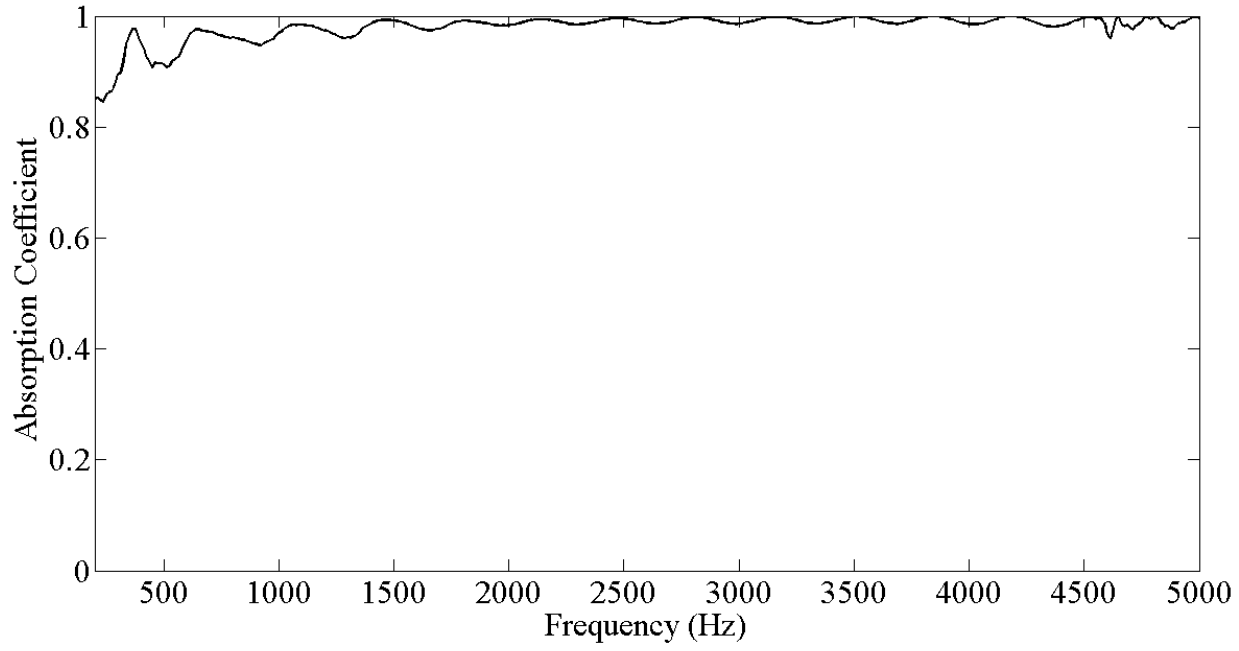

(a) Absorption Coefficient

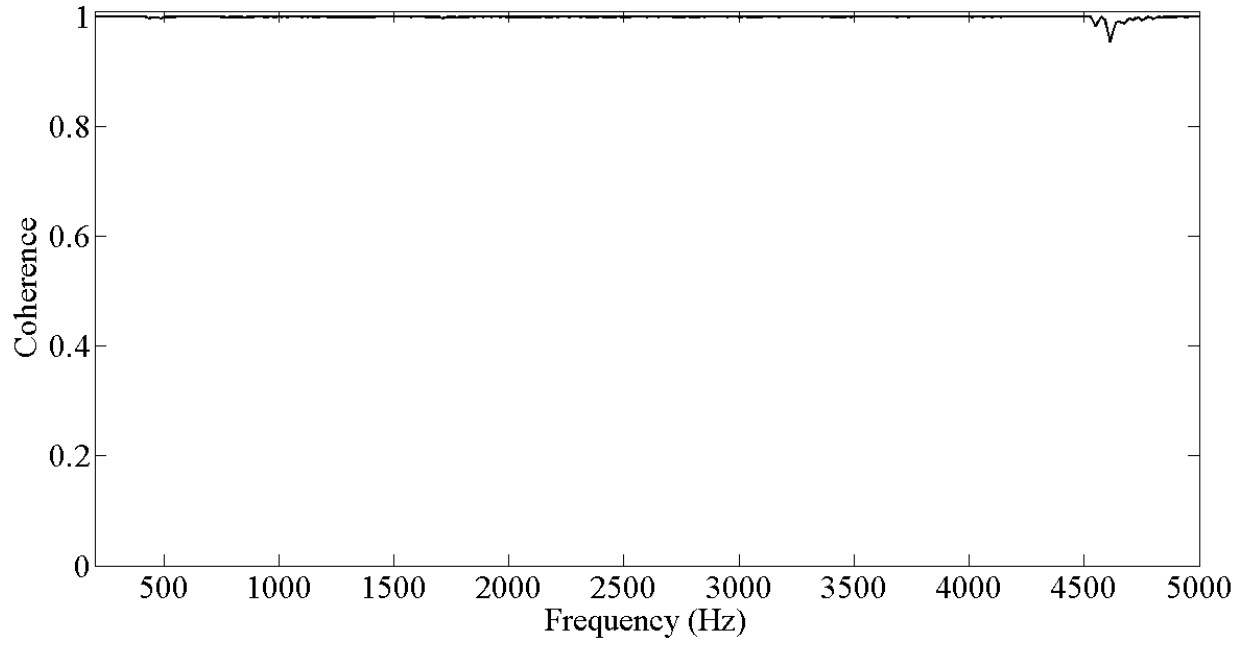

(b) Coherence

Figure 4.3: Measured absorption coefficient of anechoic foam sample. 


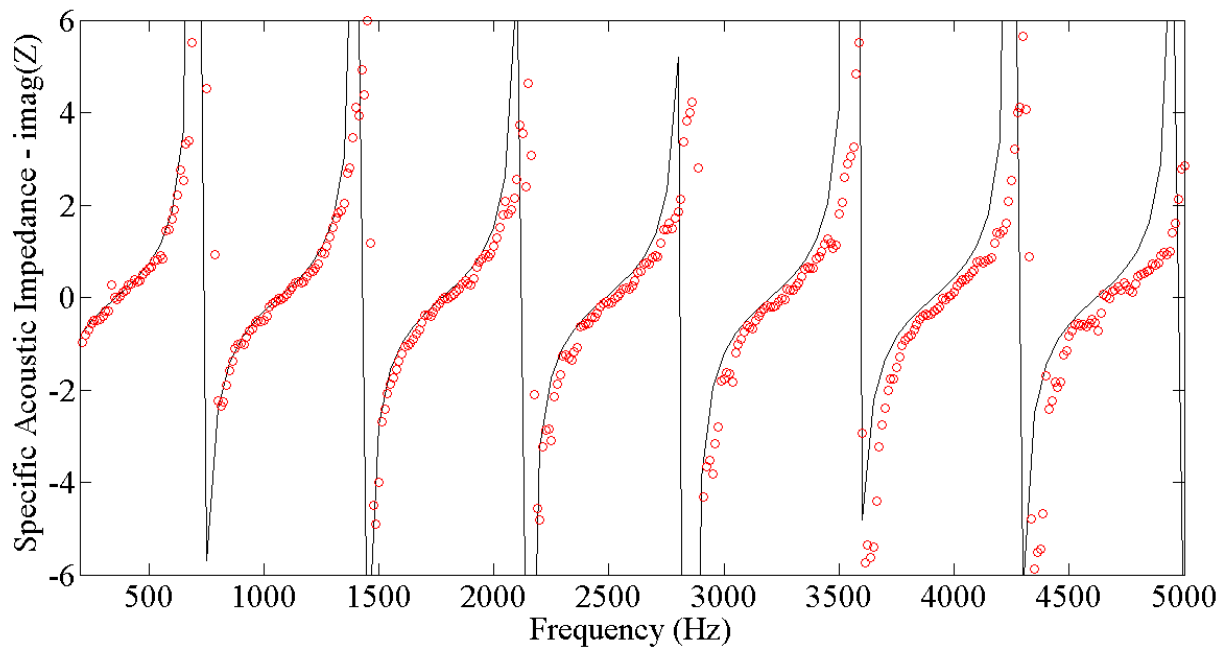

(a) Specific Acoustic Impedance

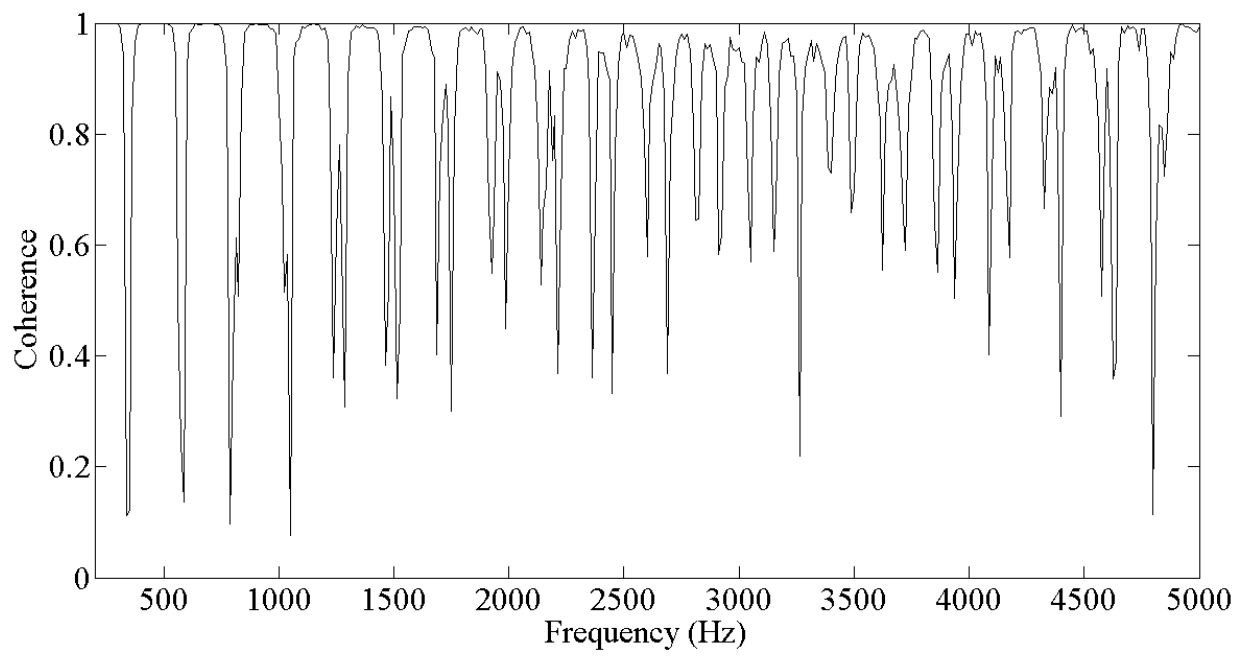

(b) Coherence

Figure 4.4: Measured specific acoustic impedance of a rigid stop. 


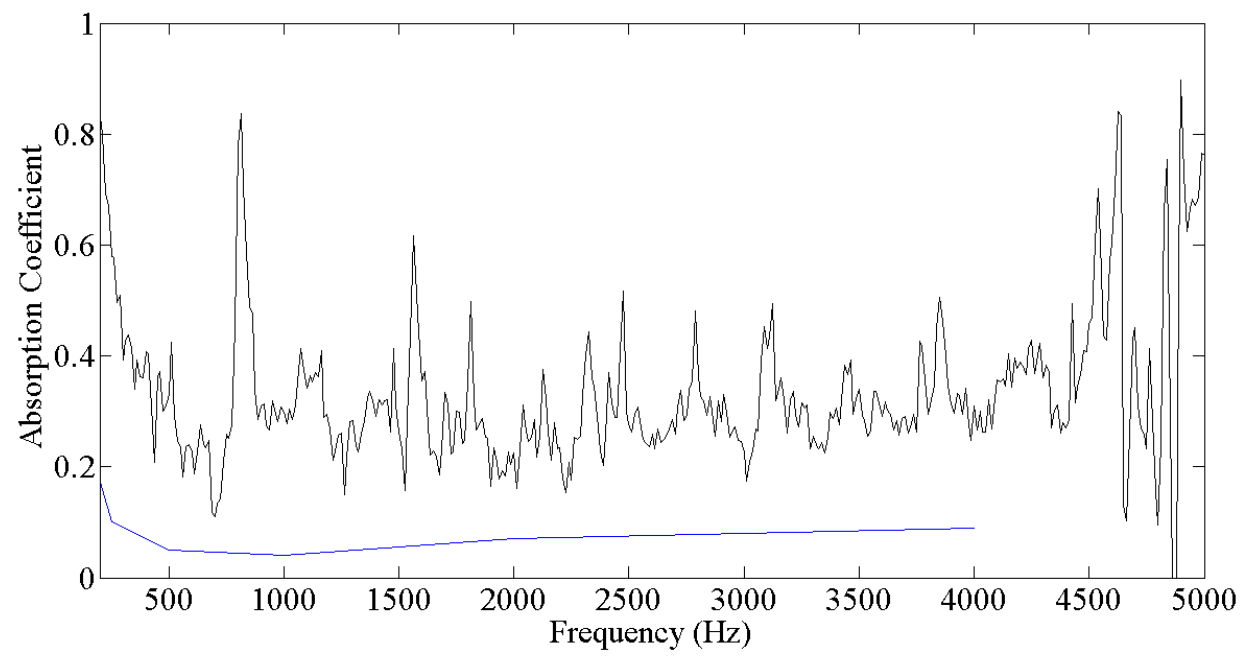

(a) Absorption Coefficient

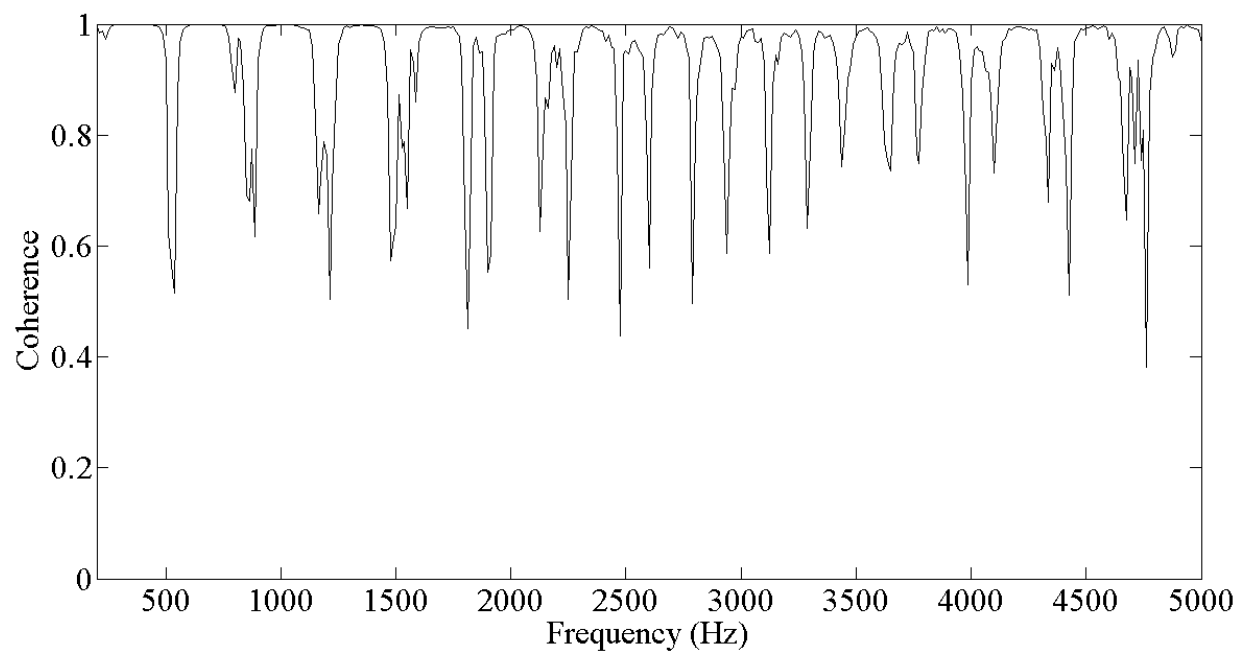

(b) Coherence

Figure 4.5: Measured absorption coefficient of gypsum wallboard sample. 


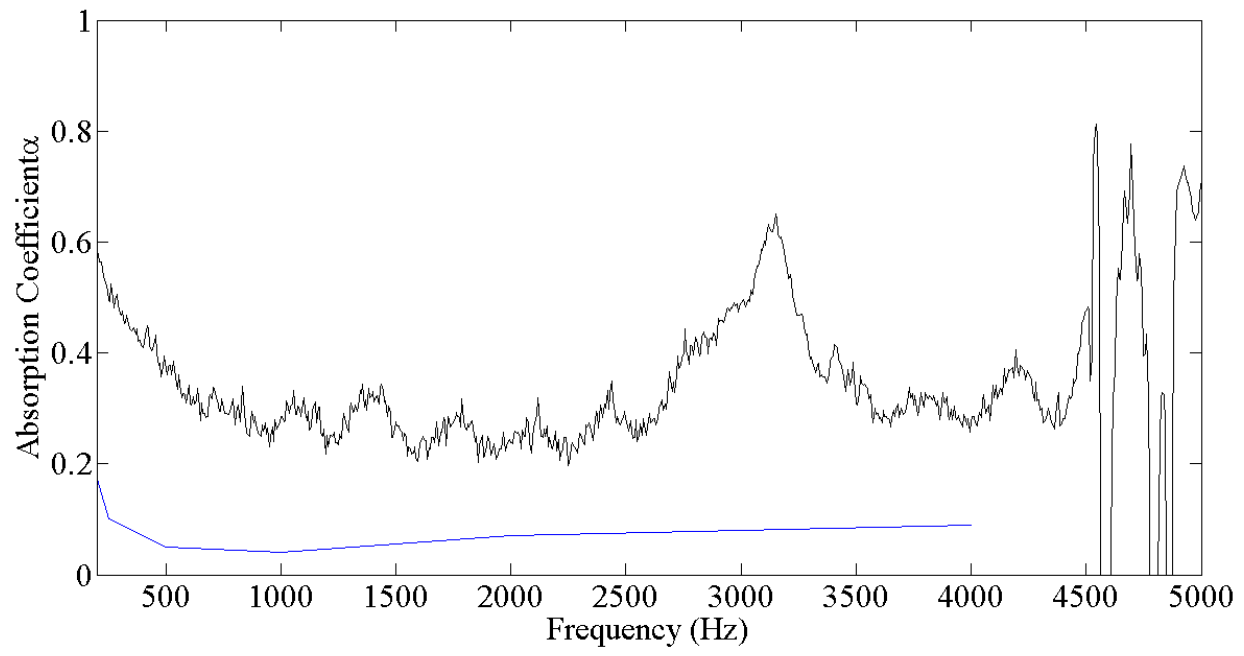

(a) Absorption Coefficient

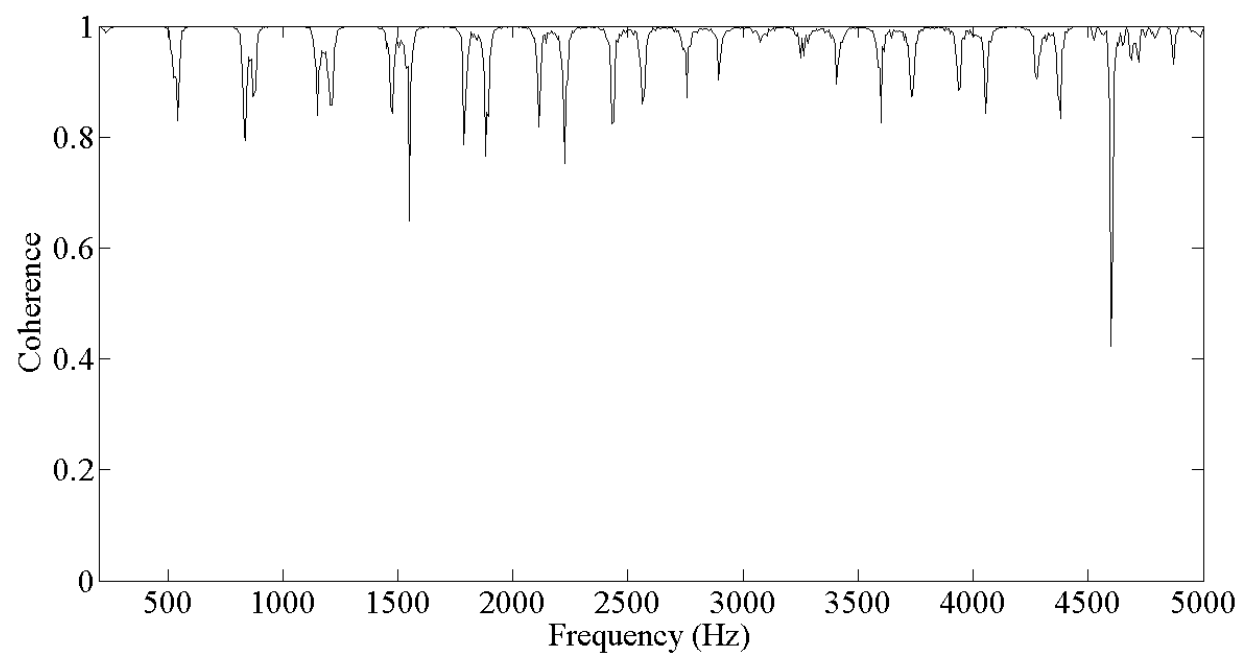

(b) Coherence

Figure 4.6: Measured absorption coefficient of gypsum wallboard sample with shorter backing plate. 


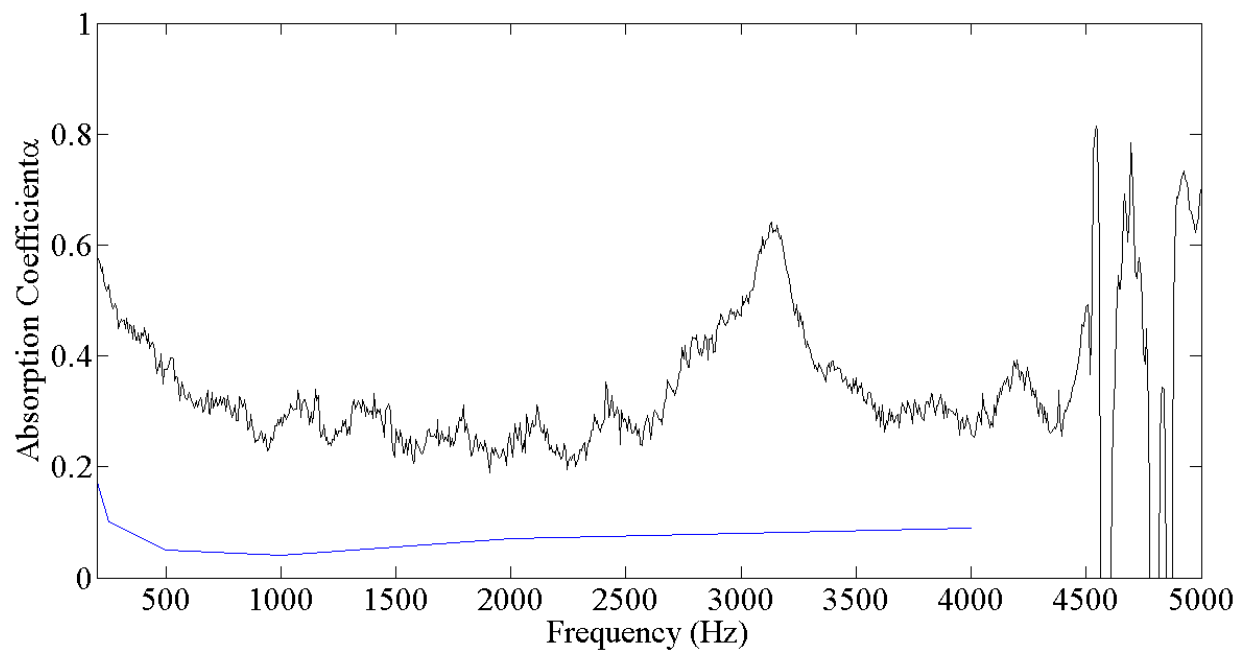

(a) Absorption Coefficient

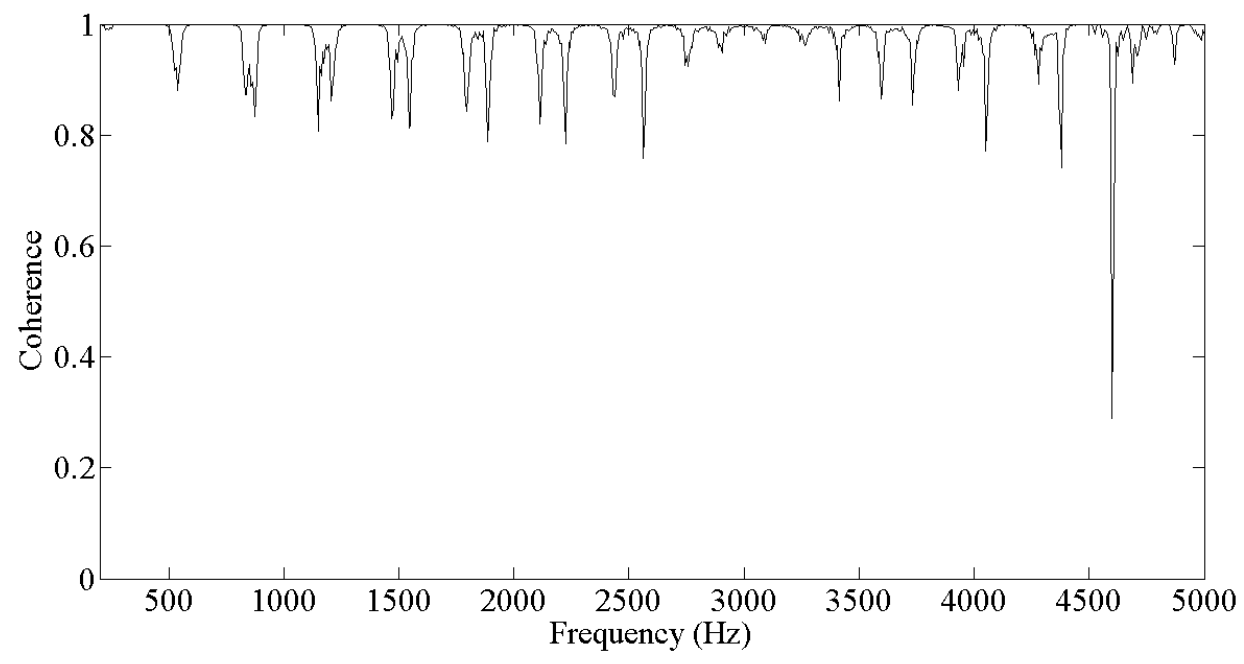

(b) Coherence

Figure 4.7: Measured absorption coefficient of burned gypsum wallboard sample. 


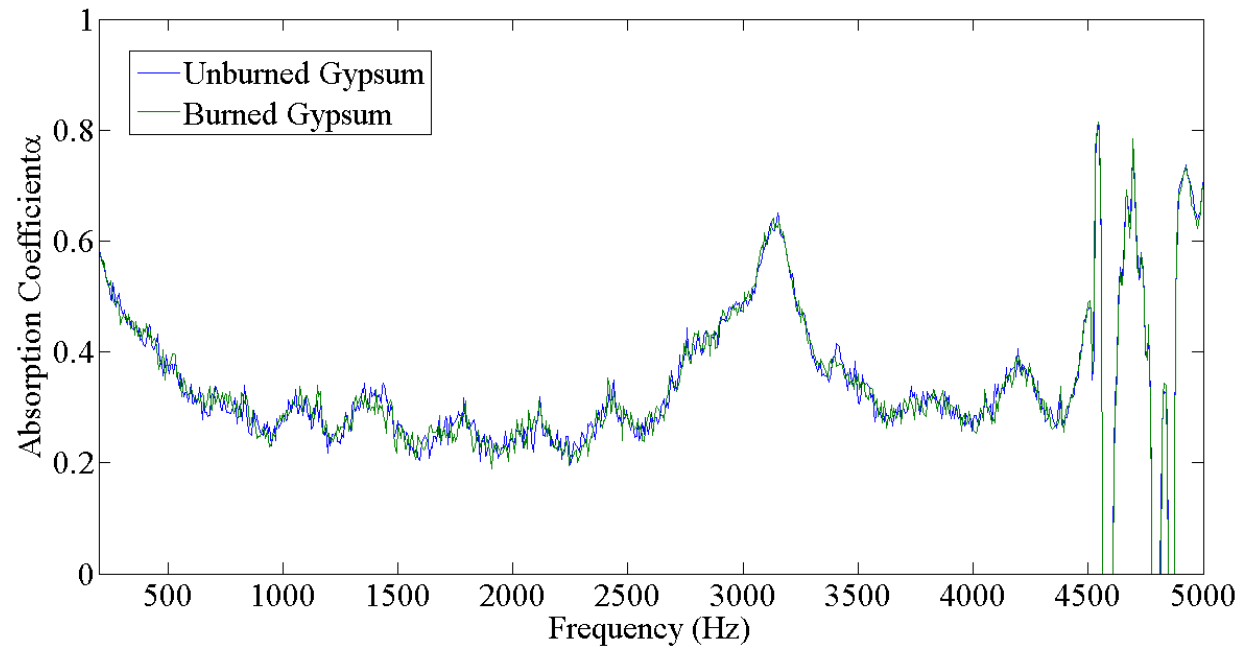

Figure 4.8: Comparison of the measured absorption coefficients of burned and unburned gypsum samples. Curves are nearly identical. 


\section{Chapter 5}

\section{Conclusion}

This report represents the integration of several years of investigation into a comprehensive analysis. Each of the components, including an acoustic analysis of firefighting equipment, the modeling and validation of sound transmission, and a physiological acoustic analysis, was described in Chapter 1. Additionally, a comprehensive review of the history of PASS devices is included.

In Chapter 2, the research team took each of these components into account in the creation of a firefighting localization test. In these tests, noise sources were placed strategically throughout various training spaces at the Austin Fire Department, the Glendale Fire Department, and the Oklahoma City Fire Department. Firefighters were then dispatched in full gear and instructed to use their standard search-and-rescue protocol to locate a sounding PASS alarm amidst the typical fireground noise. Their time and search patterns were recorded. The qualitative results of those tests, as described in this thesis, prompted the need for advanced modeling efforts.

In Chapter 3, several modeling methods that might be used to evaluate the spaces of interest in the localization tests are described. Finite element 
modeling was selected as the most efficient option, and a full example of that method is provided. Future work will allow for both the integration of many noise sources into a single comprehensive sound field to be compared to the sound field for the PASS device and the auralization of the resultant signal at a given location. These results will be critical for the integration of noise into firefighter training protocols; with a comprehensive model, future research teams will be able to identify potentially confusing situations for implementation in training. With this exposure and the associated improvement in training, firefighters will be better equipped for search and rescue situations in actual fire scenarios. 
Appendices 


\section{Appendix A}

\section{MATLAB Code for COMSOL Model of AFD Office Building}

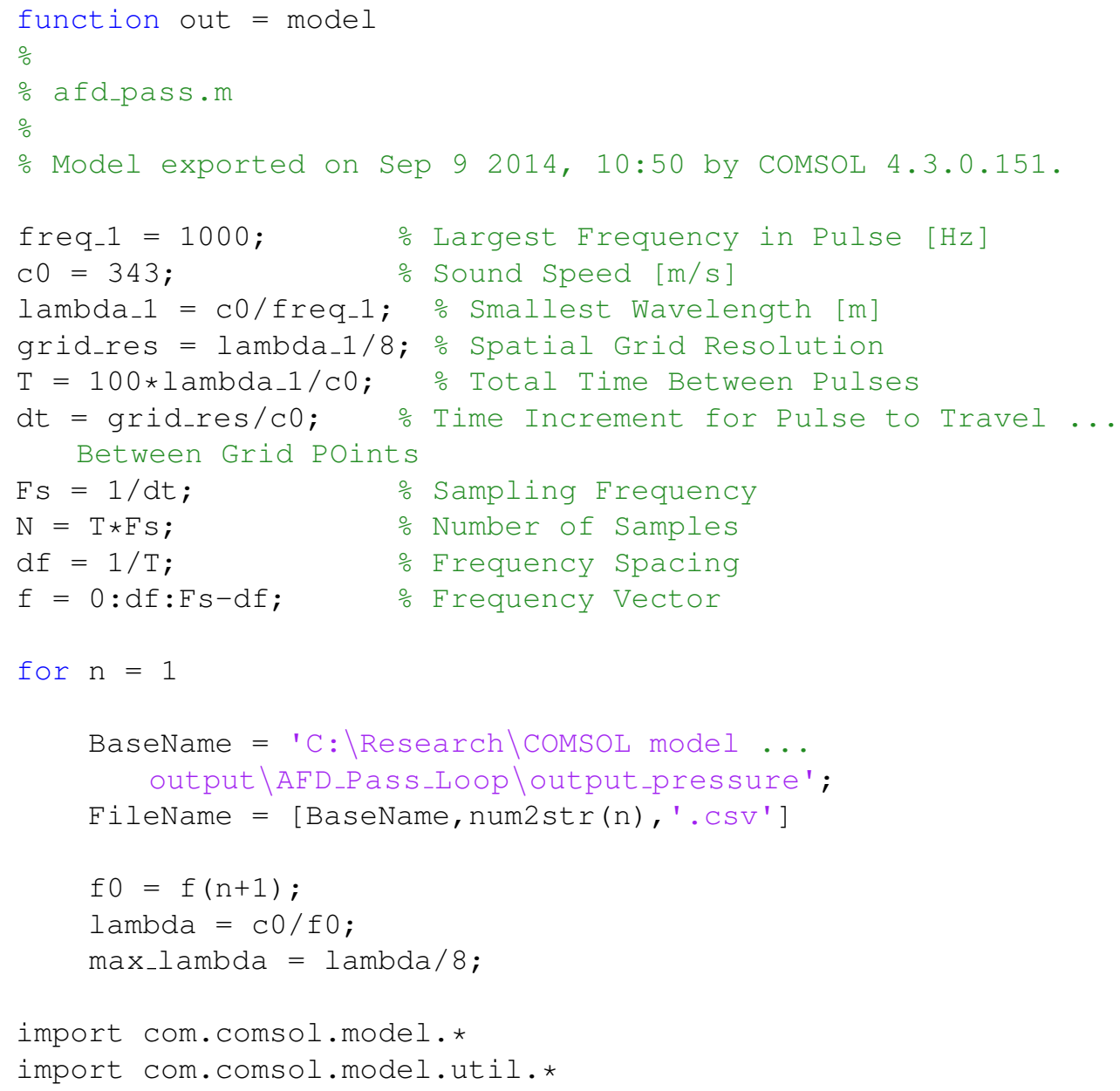




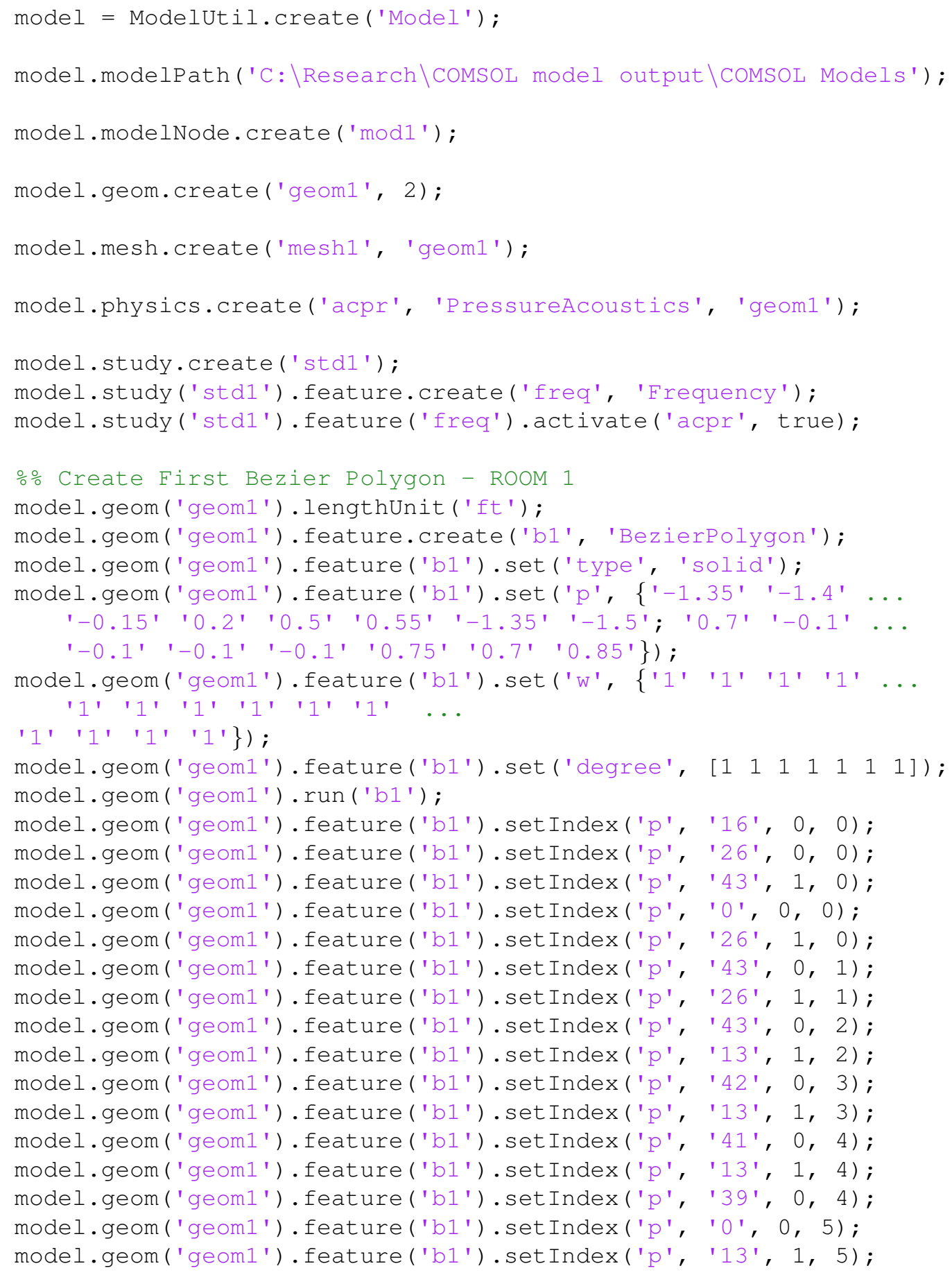




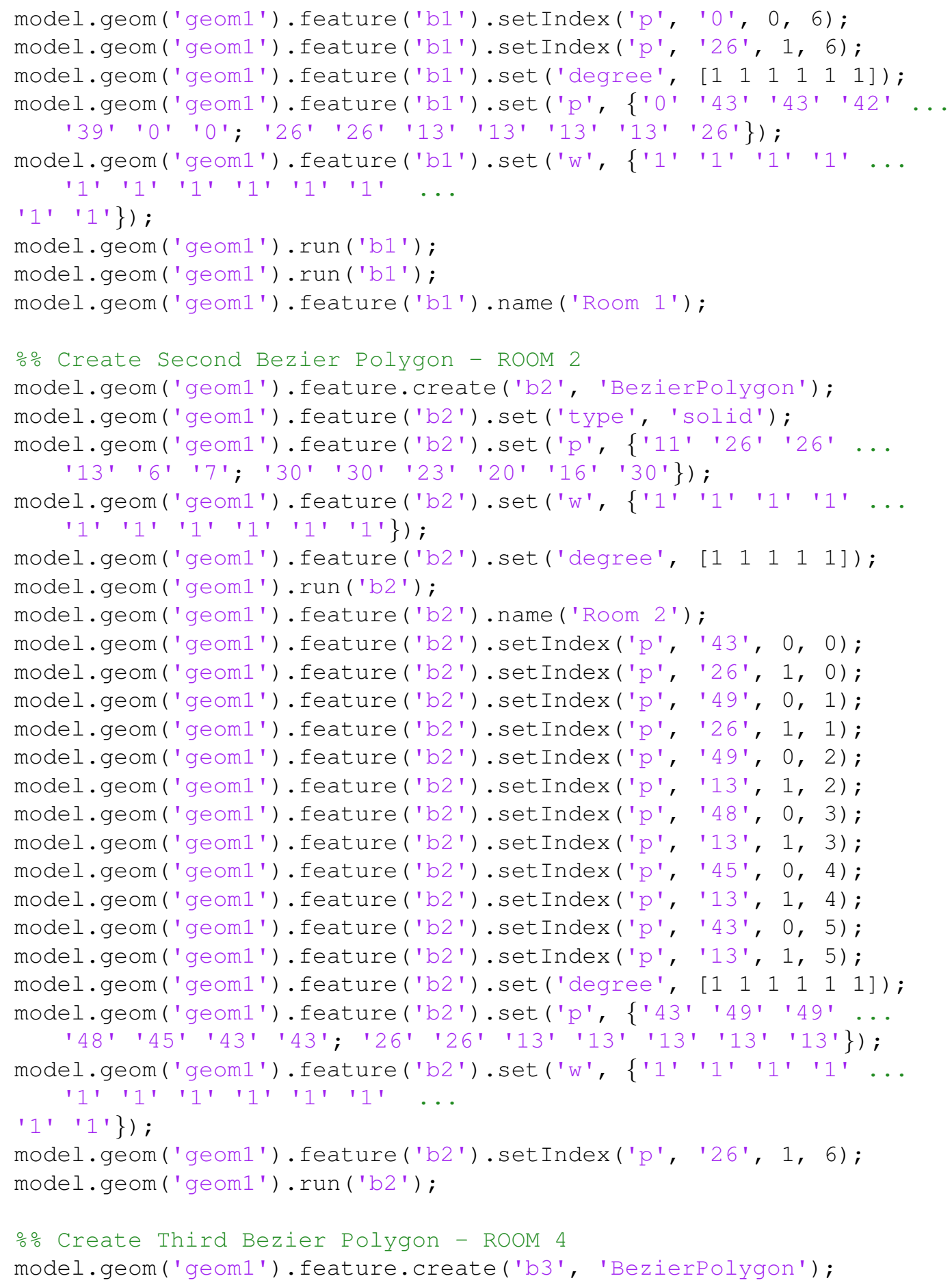




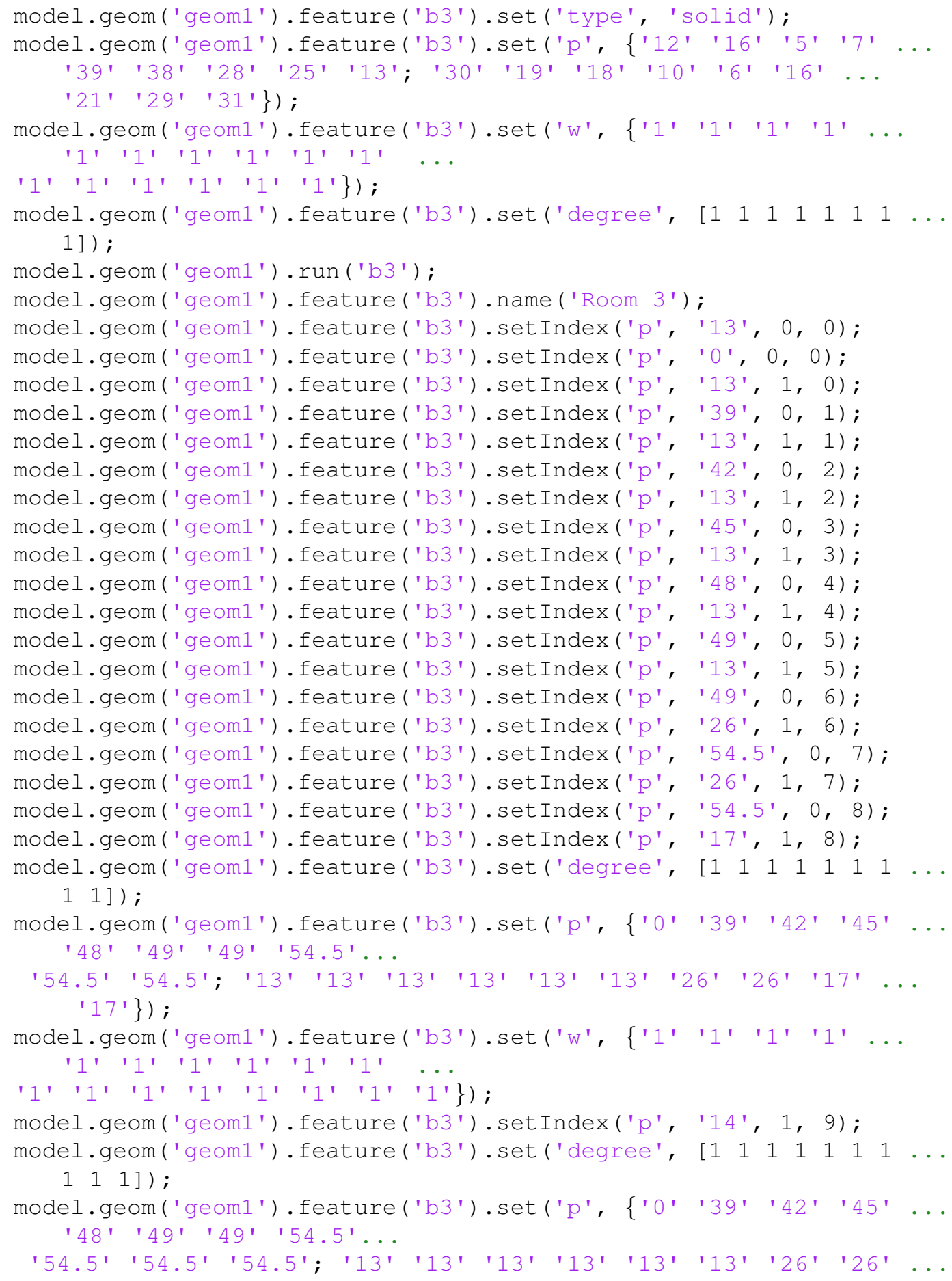




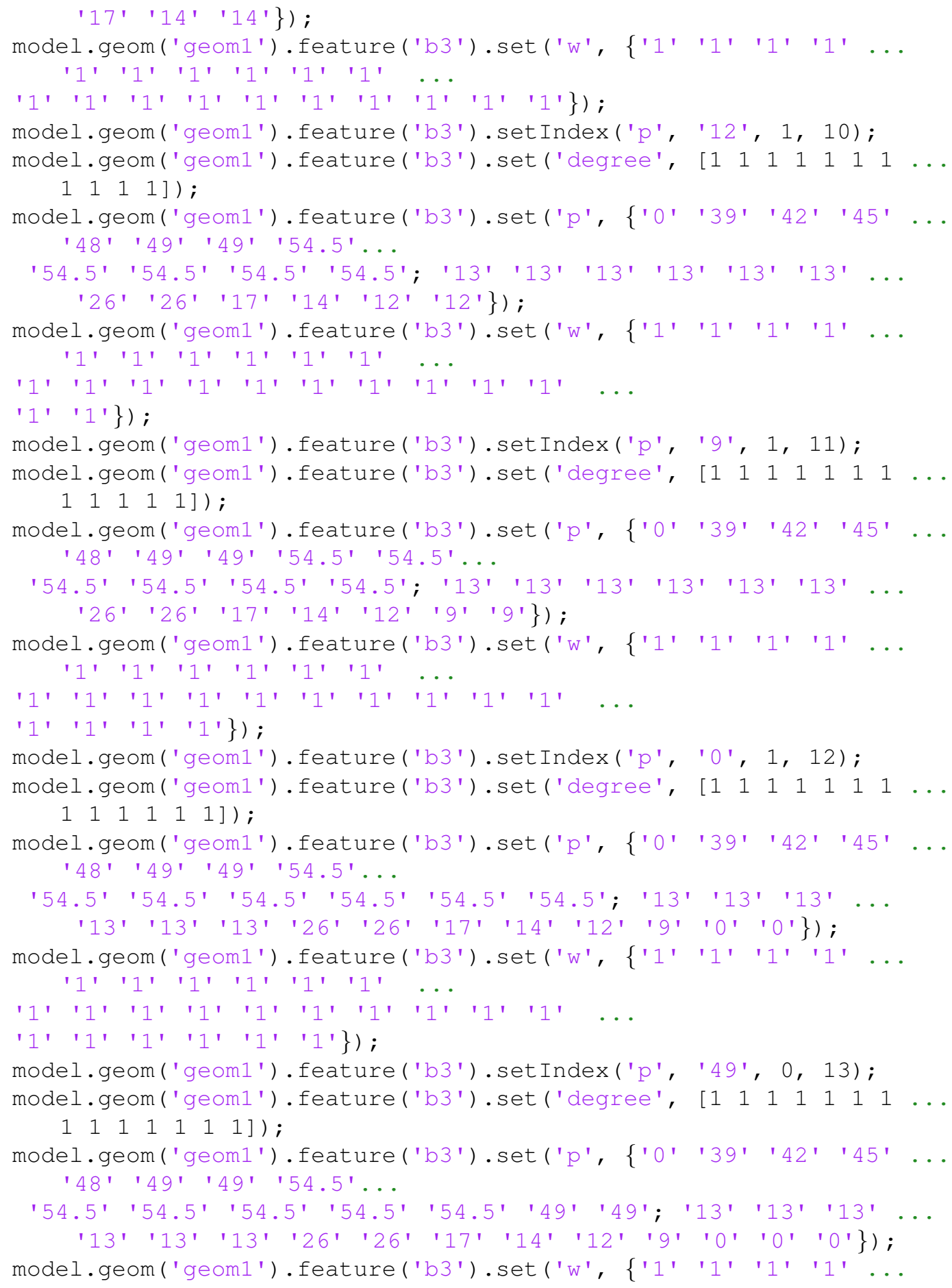




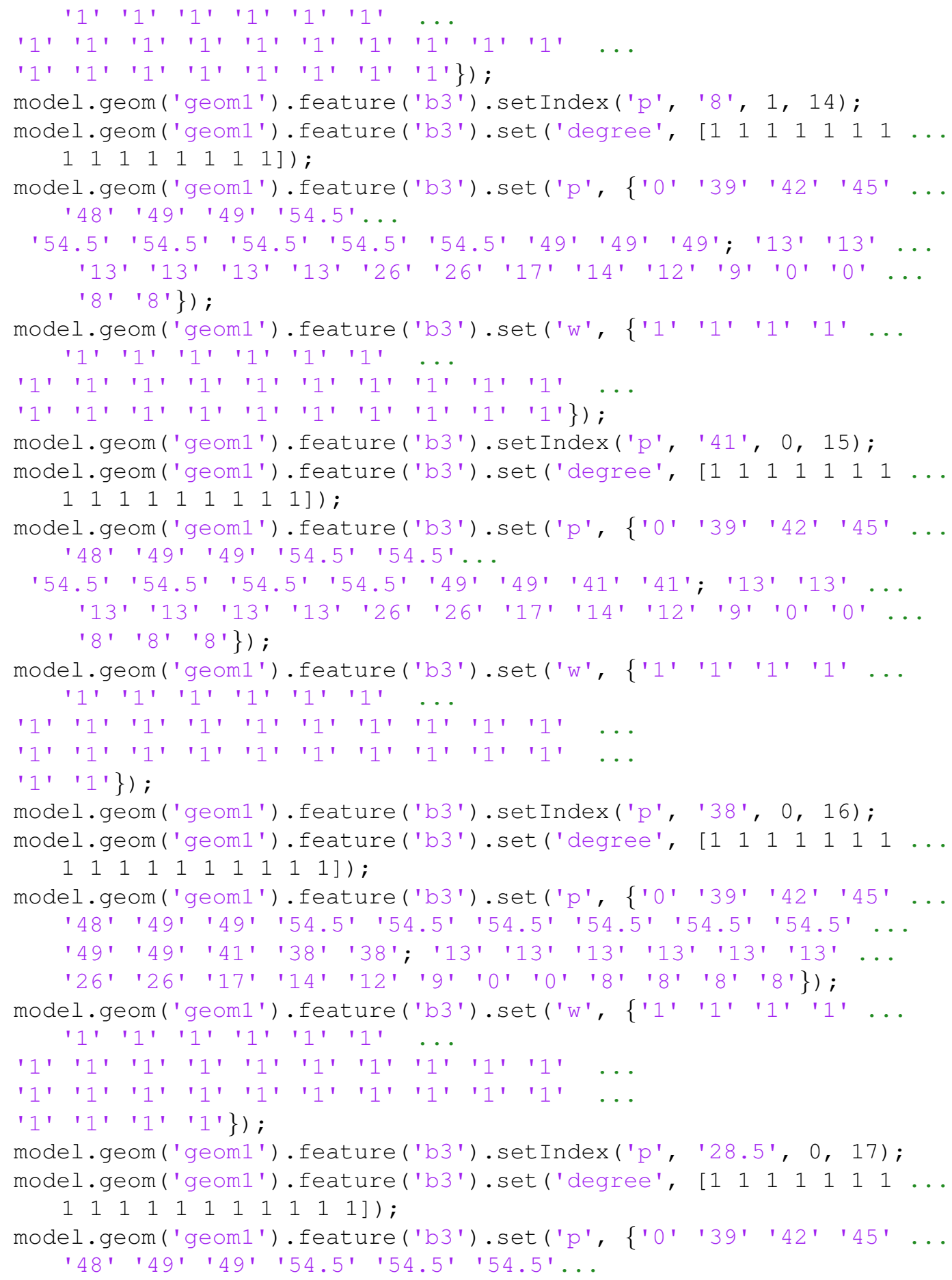




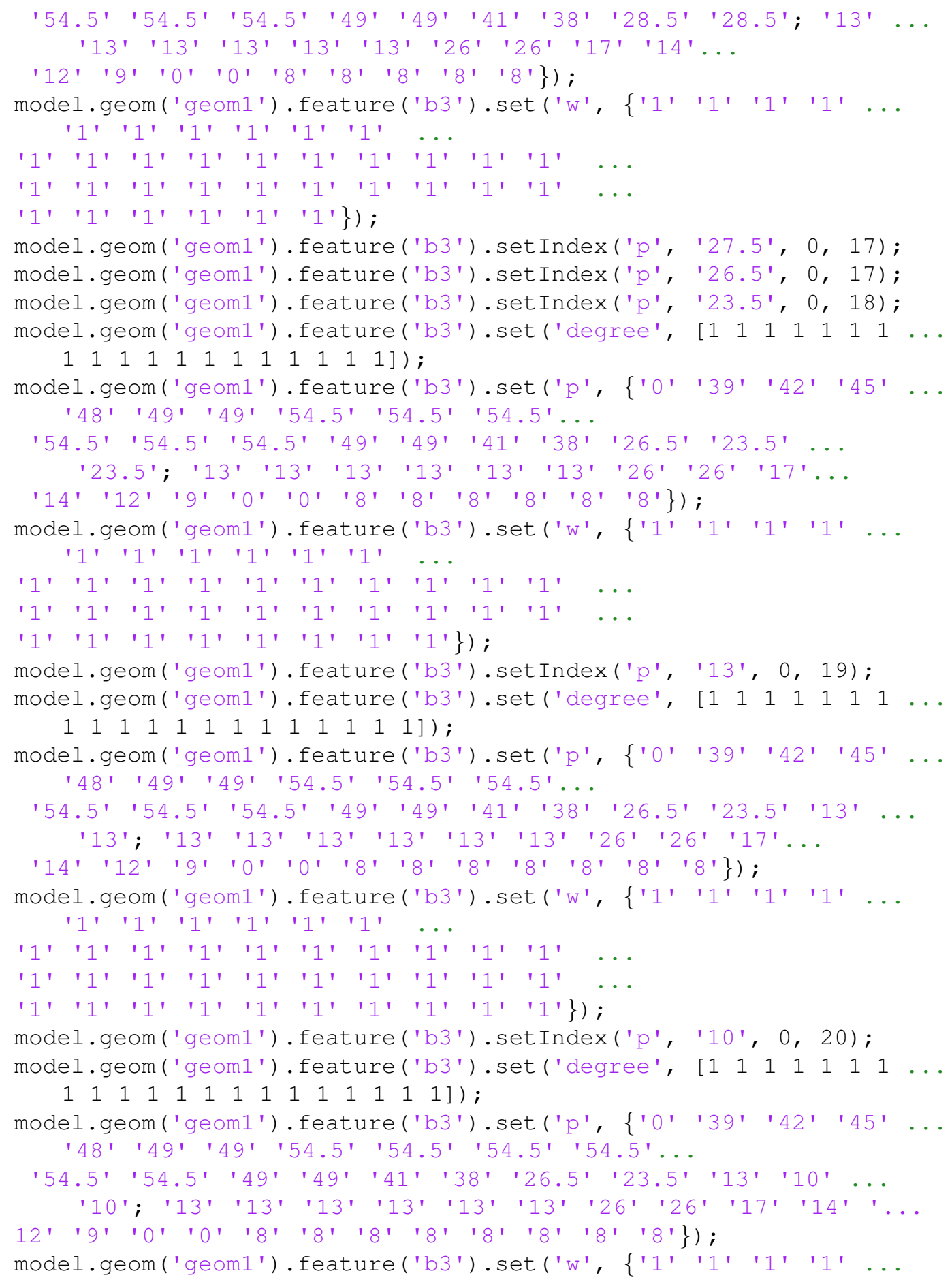




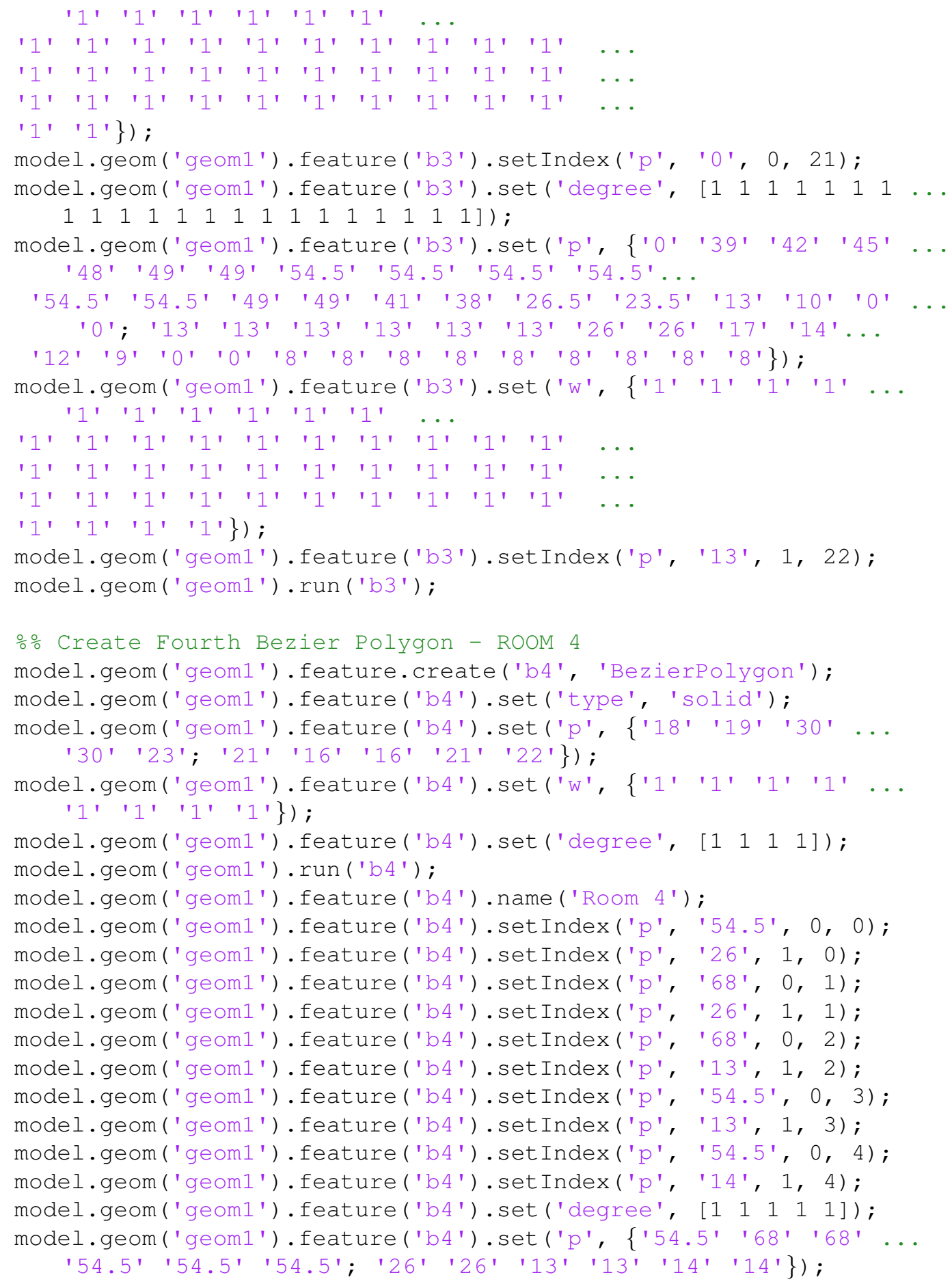




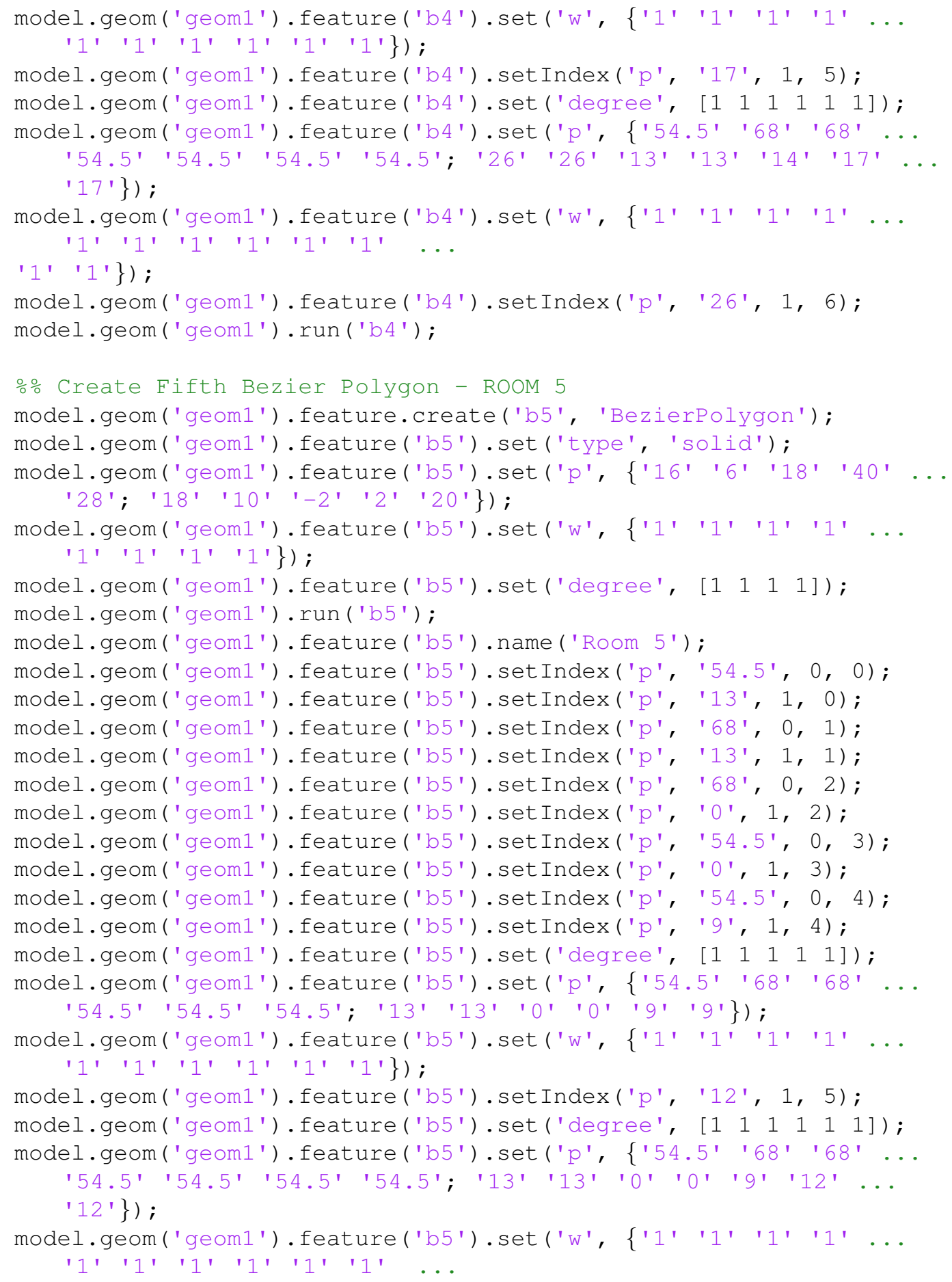




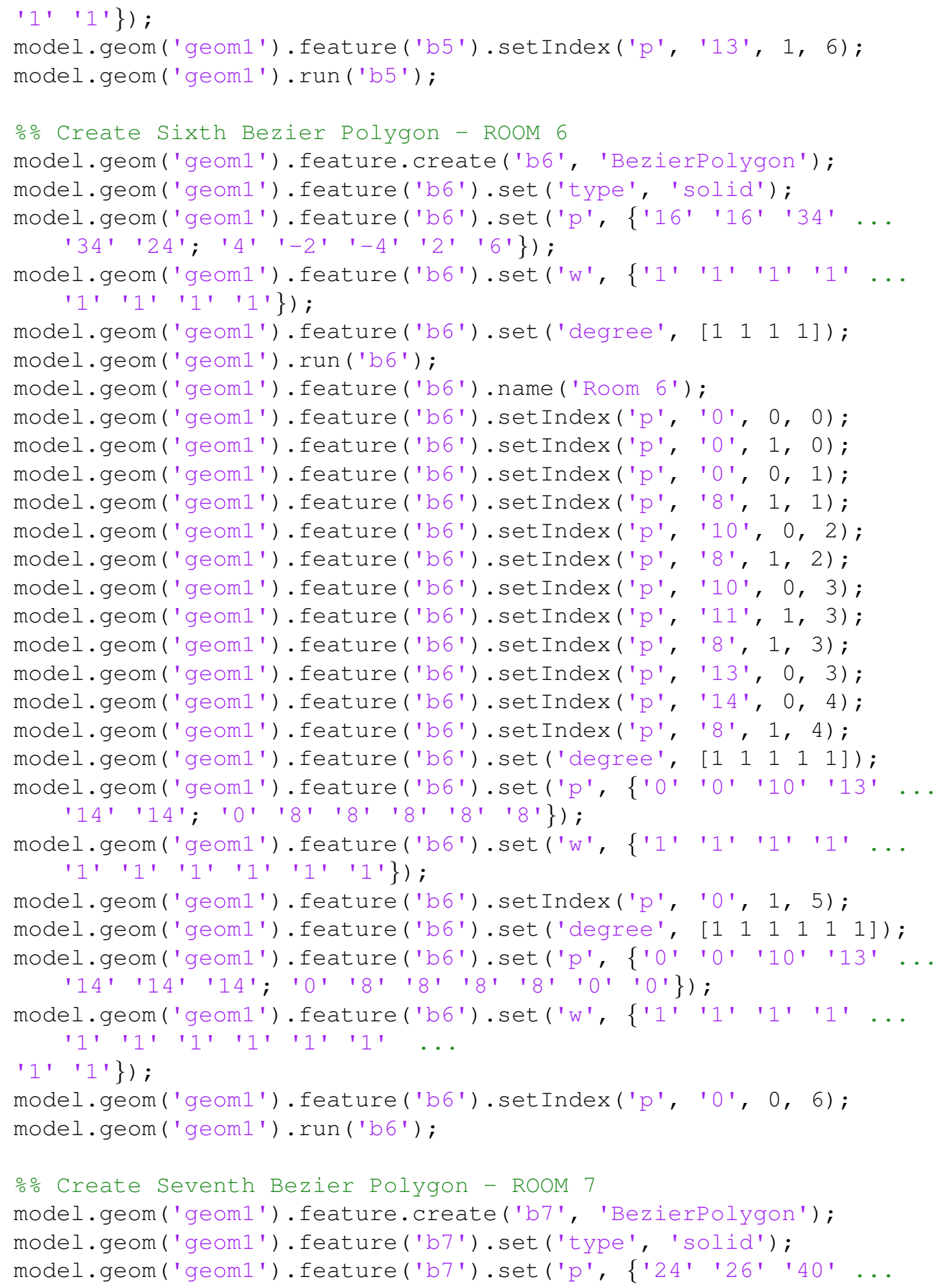




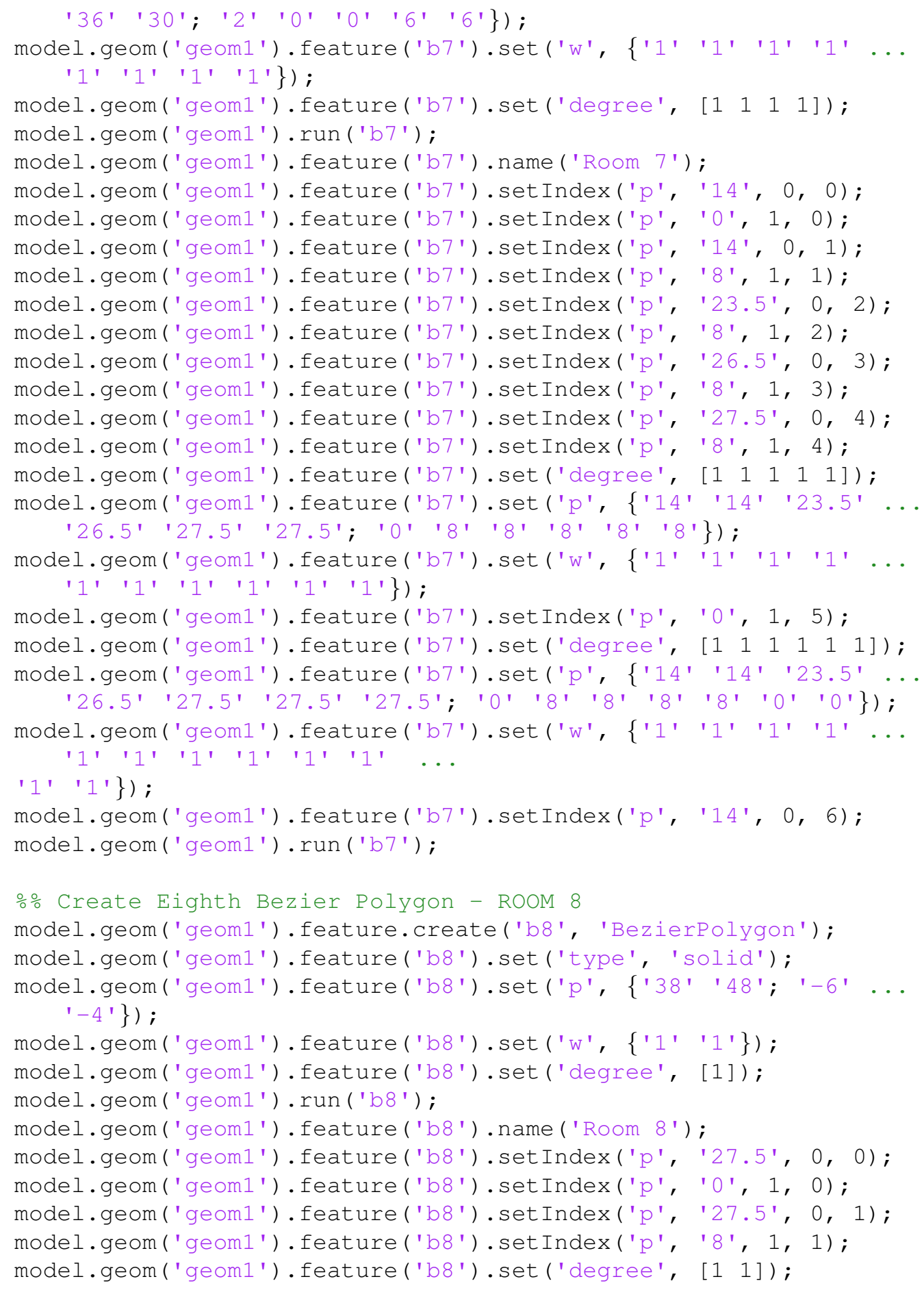




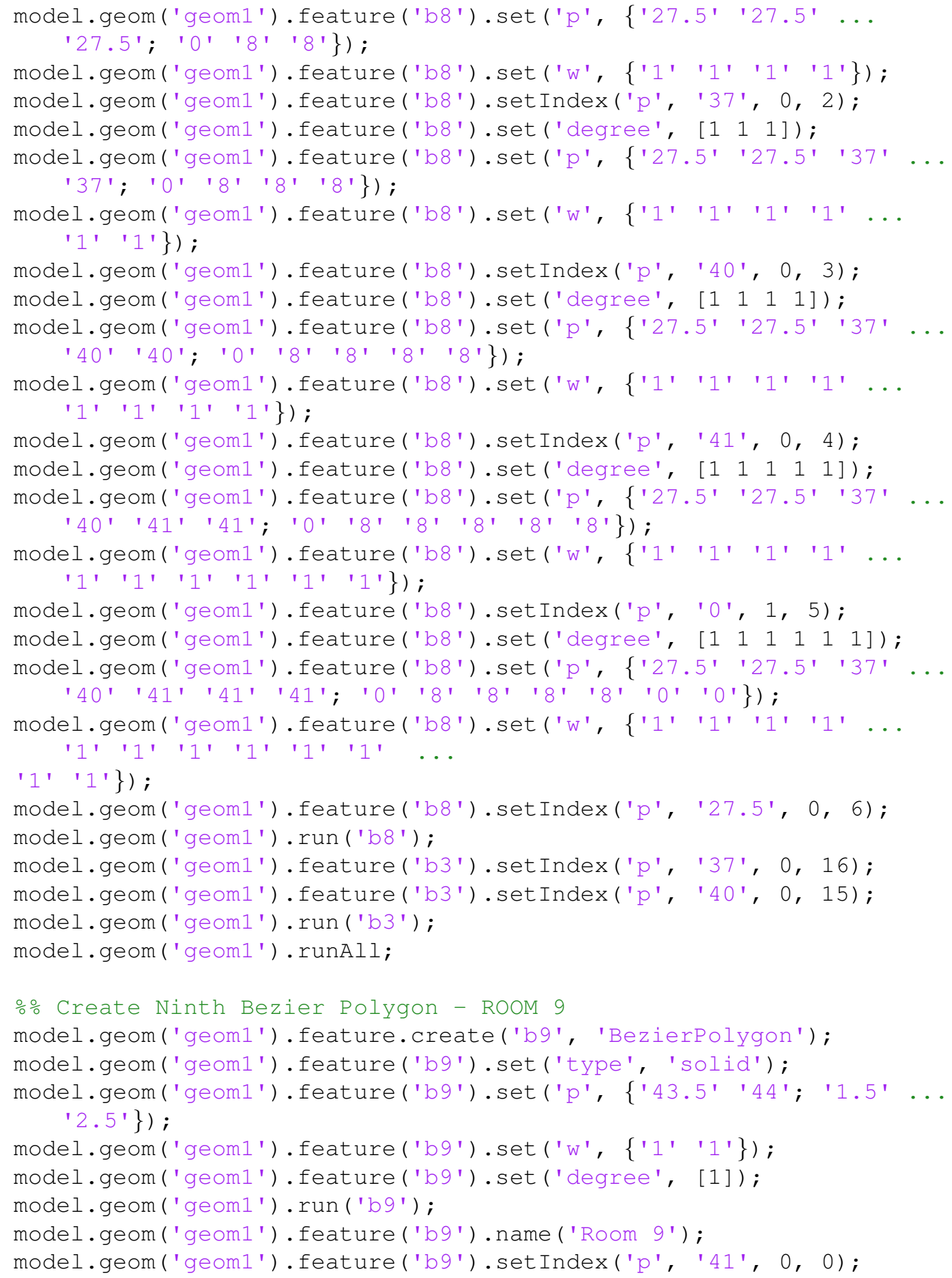




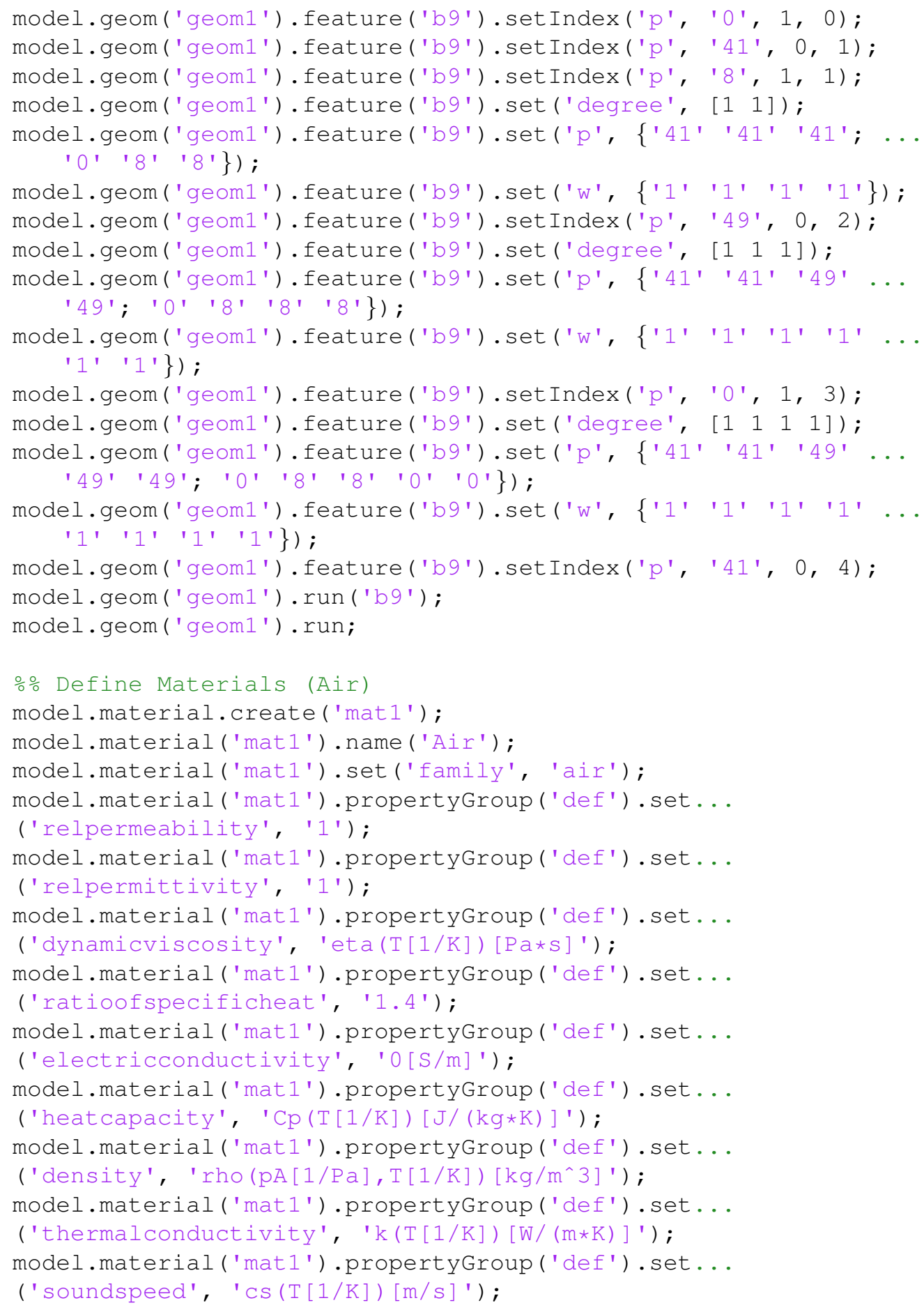


model.material ('mat1').propertyGroup ('def').func.create ('eta', ...

'Piecewise') ;

model.material ('mat1').propertyGroup ('def').func('eta').set ('funcname', ...

'eta');

model.material ('mat1') .propertyGroup ('def').func ('eta' ). set ('arg', ...

' $T$ ') ;

model.material ('mat1') .propertyGroup ('def ').func ('eta' ).set ('extrap', ...

'constant');

model.material ('mat1') .propertyGroup ('def').func('eta' ).set ('pieces ', ...

$\{$ '200.0' '1600.0' ...

$'-8.38278 \mathrm{E}-7+8.35717342 \mathrm{E}-8 * \mathrm{~T}^{\wedge} 1-7.69429583 \mathrm{E}-11 * \mathrm{~T}^{\wedge} 2+\ldots$

$\left.\left.4.6437266 \mathrm{E}-14 * \mathrm{~T}^{\wedge} 3-1.06585607 \mathrm{E}-17 * \mathrm{~T}^{\wedge} 4^{\prime}\right\}\right)$;

model.material ('mat1').propertyGroup ('def').func.create ('Cp', ...

'Piecewise');

model.material ('mat1').propertyGroup ('def').func ('Cp'). set ('funcname', ... 'Cp');

model.material ('mat1').propertyGroup ('def').func ('Cp').set ('arg', ... 'T');

model.material ('matl').propertyGroup ('def').func('Cp').set ('extrap', ... 'constant');

model.material ('mat1').propertyGroup ('def').func('Cp').set ('pieces', ...

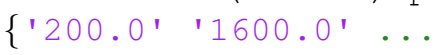

' $1047.63657-0.372589265 * \mathrm{~T}^{\wedge} 1+9.45304214 \mathrm{E}-4 * \mathrm{~T}^{\wedge} 2-\ldots$

$\left.\left.6.02409443 \mathrm{E}-7 * \mathrm{~T}^{\wedge} 3+1.2858961 \mathrm{E}-10 * \mathrm{~T}^{\wedge} 4^{\prime}\right\}\right) ;$

model.material ('mat1').propertyGroup ('def'). func.create ('rho', ...

'Analytic');

model.material ('mat1').propertyGroup ('def').func ('rho').set ('funcname', ... 'rho');

model.material ('matl').propertyGroup ('def'). func ('rho'). set ('args', ... $\{$ 'pA' ' $\mathrm{T}$ '\});

model.material ('mat1').propertyGroup ('def'). func('rho'). set ('expr', ... 'pA*0.02897/8.314/T');

model.material ('mat1') .propertyGroup ('def ').func ('rho').set ('dermethod', ... 'manual');

model.material ('mat1').propertyGroup ('def').func ('rho').set ('argders', ... $\left\{\mathrm{pA}^{\prime}\right.$ 'd $(\mathrm{pA} * 0.02897 / 8.314 / \mathrm{T}, \mathrm{pA})$ '; 'T' $\ldots$

' d ( $\left.\left.\mathrm{pA} * 0.02897 / 8.314 / \mathrm{T}, \mathrm{T}){ }^{\prime}\right\}\right) ;$

model.material ('matl').propertyGroup ('def').func.create ('k', ...

'Piecewise') ;

model.material ('mat1') .propertyGroup ('def ').func ('k' ).set ('funcname', ... 'k');

model.material ('mat1') .propertyGroup ('def').func ('k').set ('arg', ... ' $\mathrm{T}$ ') ;

model.material ('matl').propertyGroup ('def'). func ('k'). set ('extrap', ...

'constant'); 


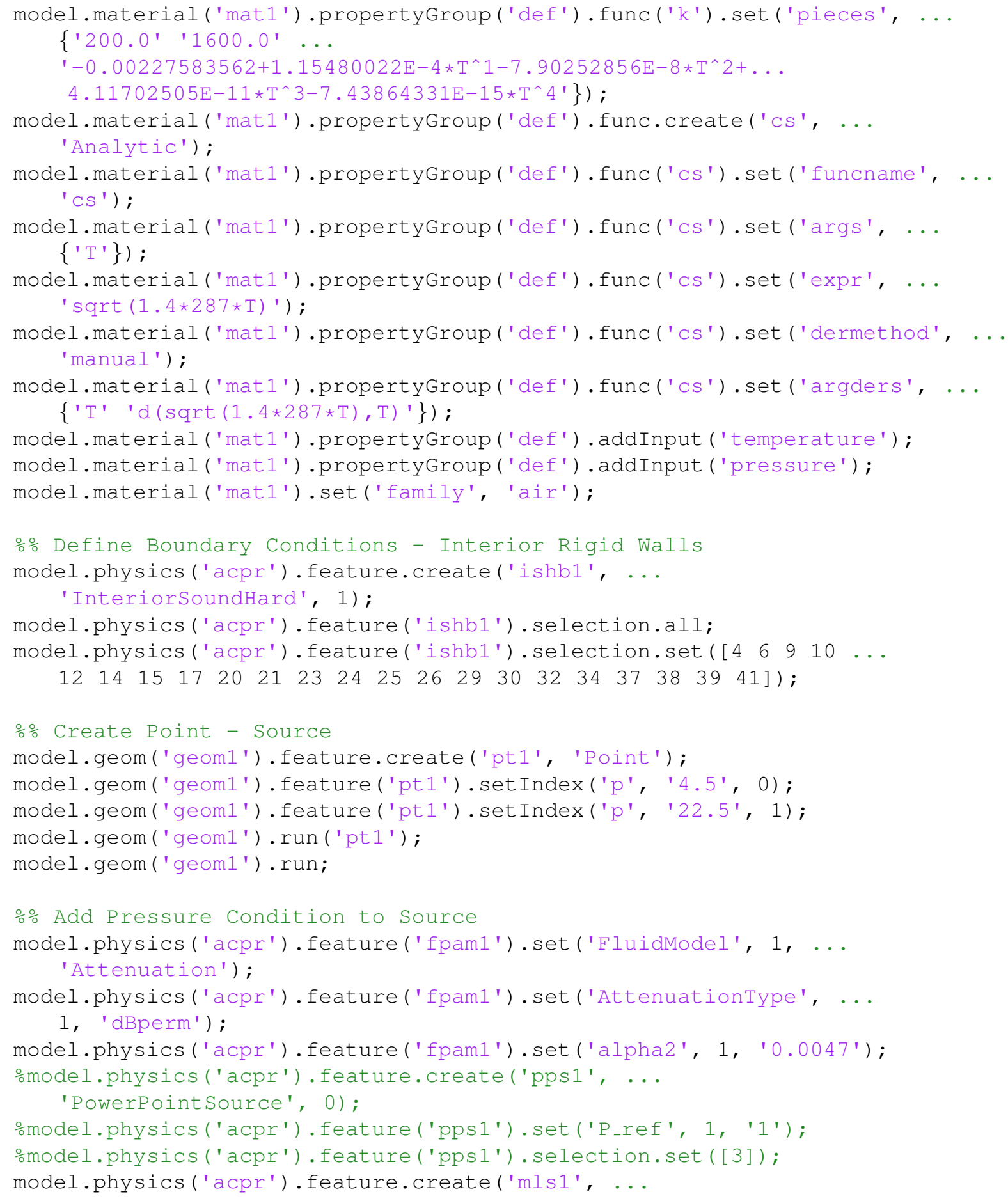




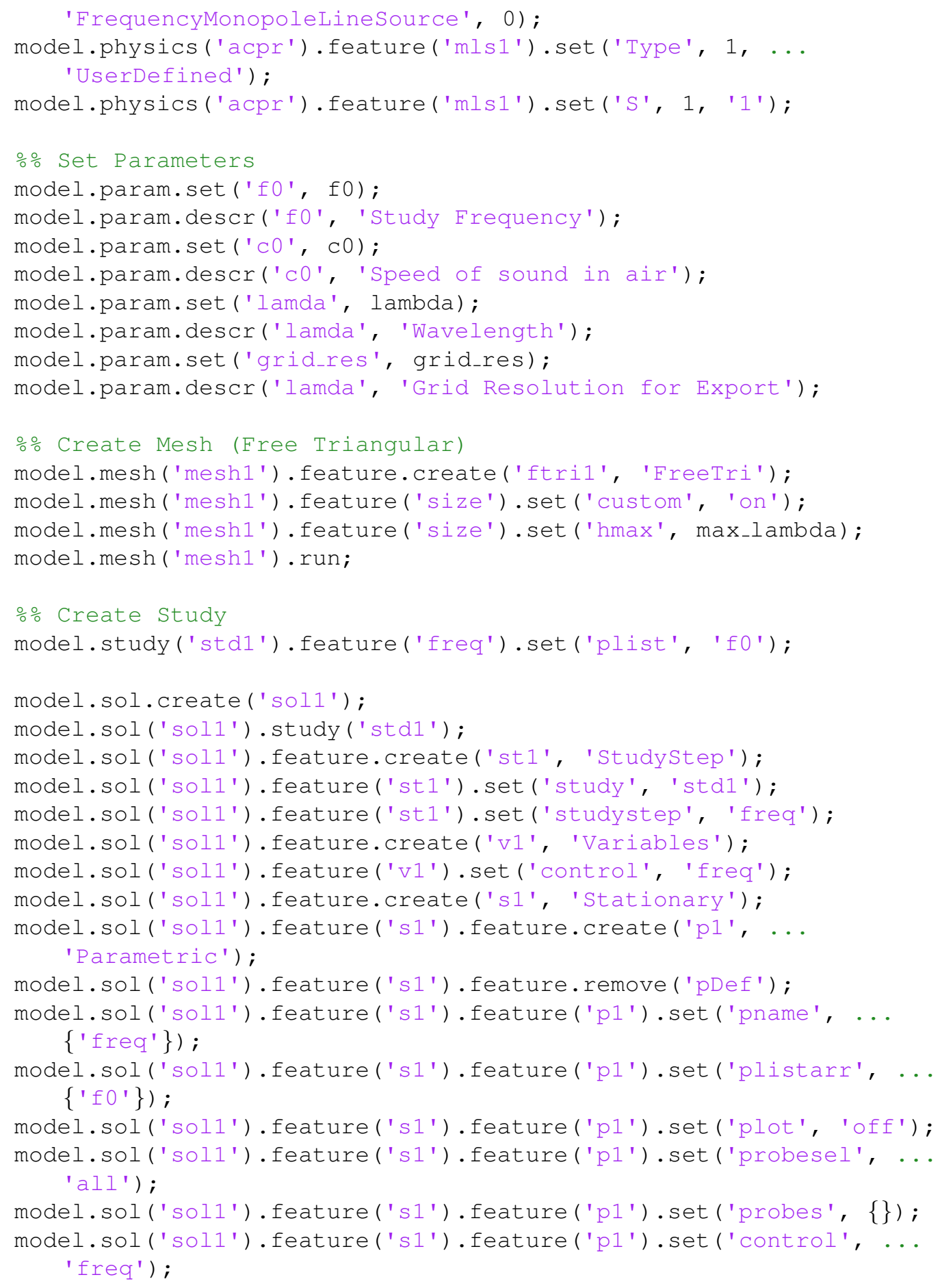




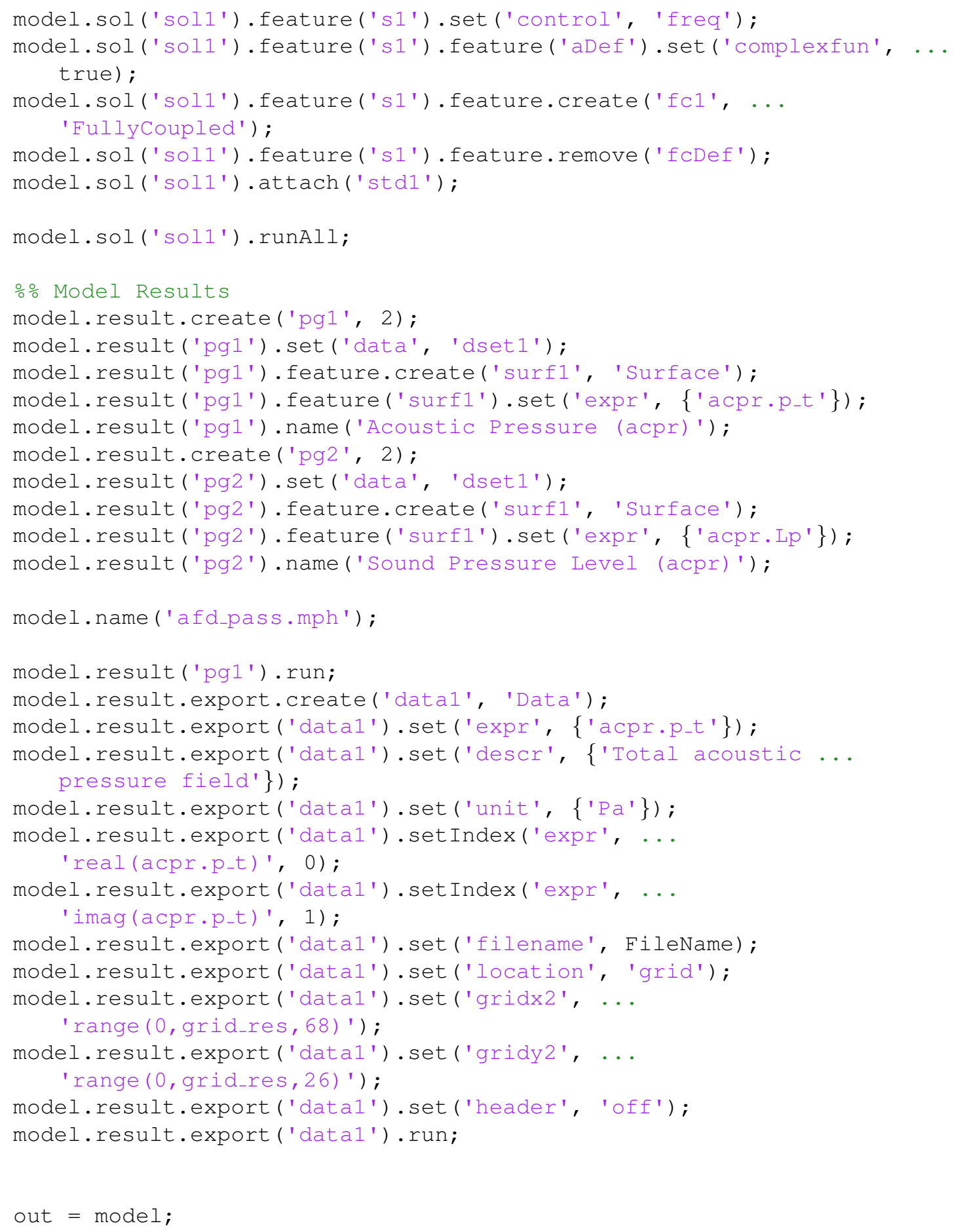


end 


\section{Appendix B}

\section{MATLAB Code for Fourier Synthesis of AFD Office Building}

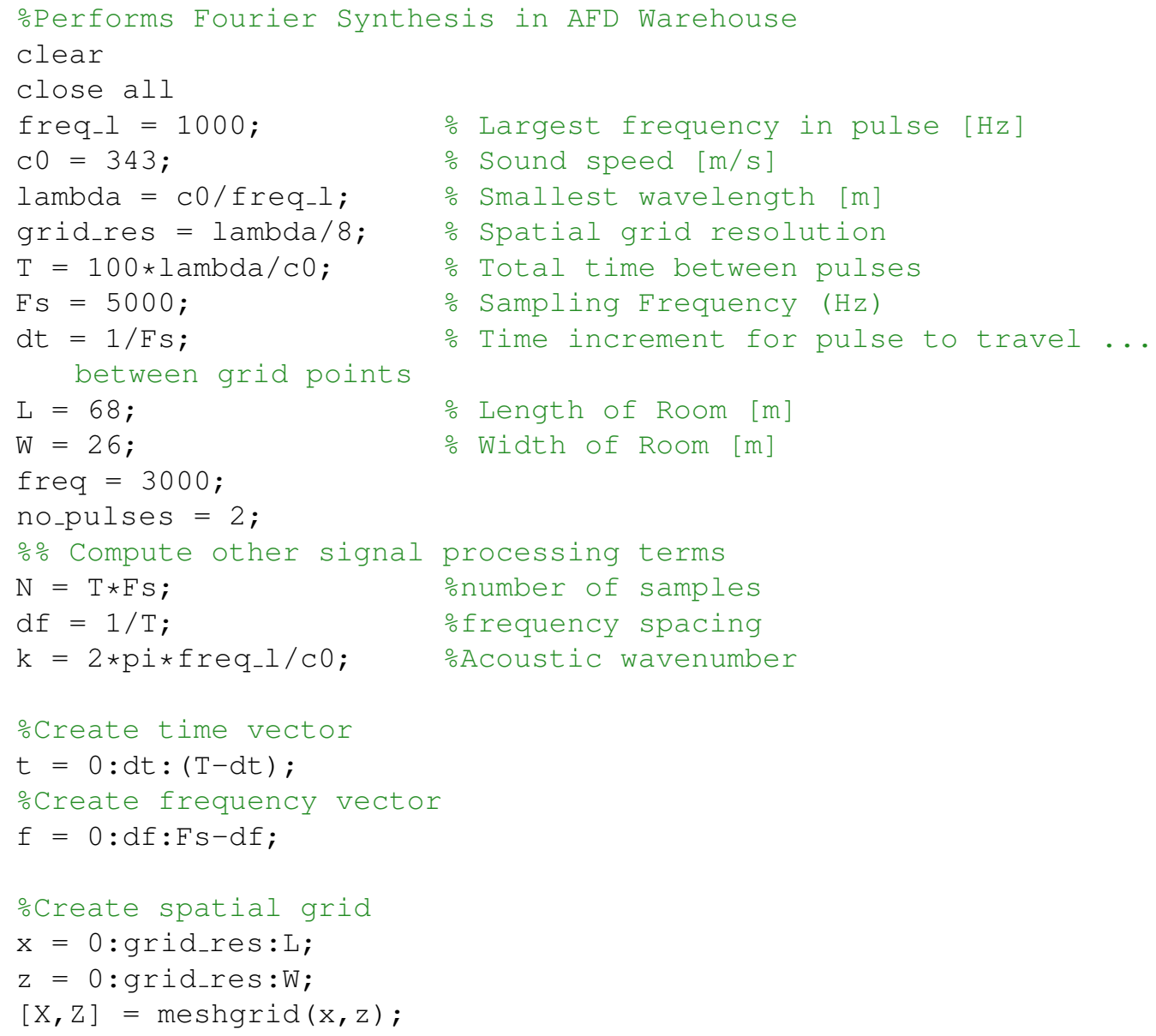




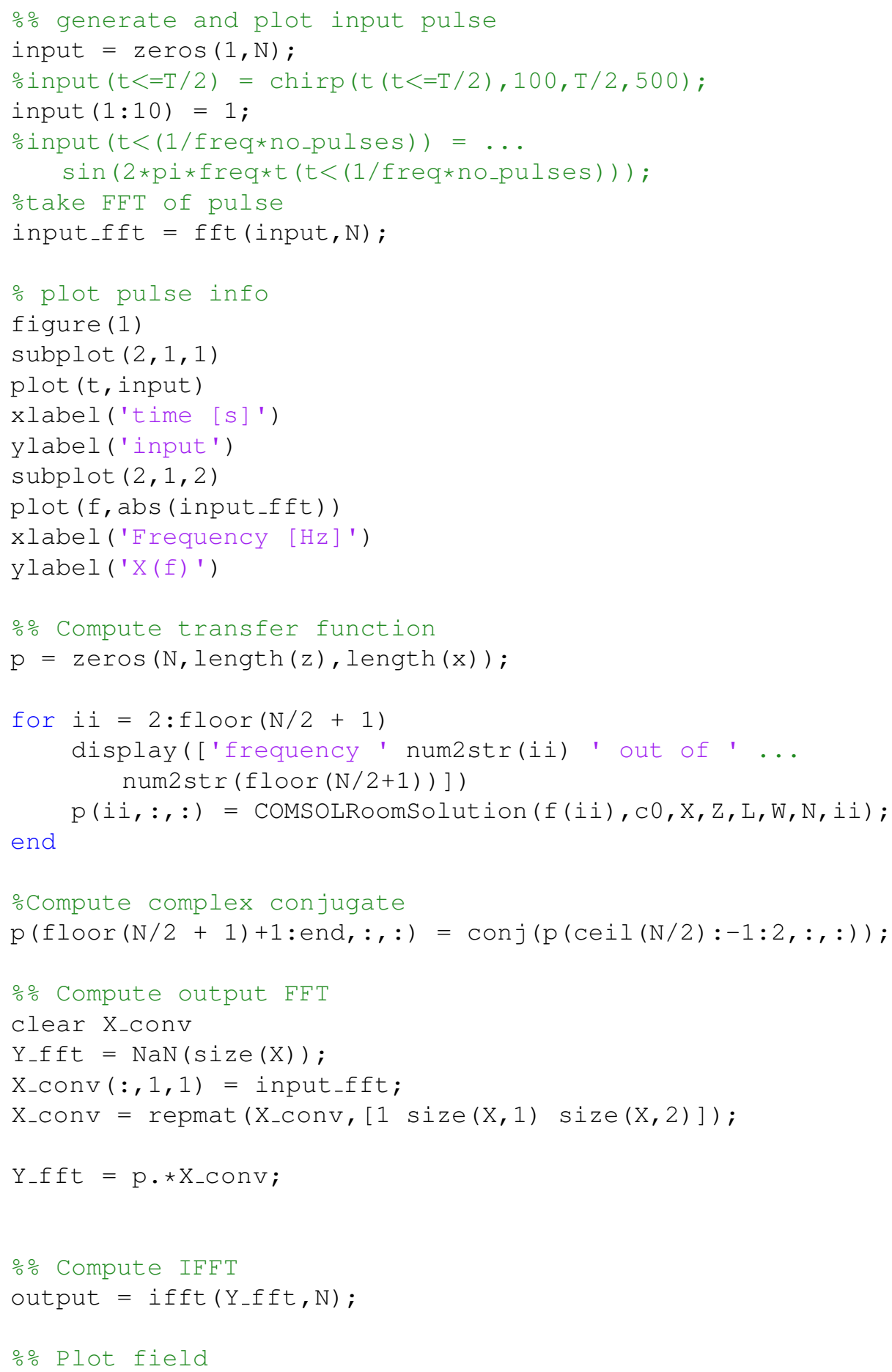




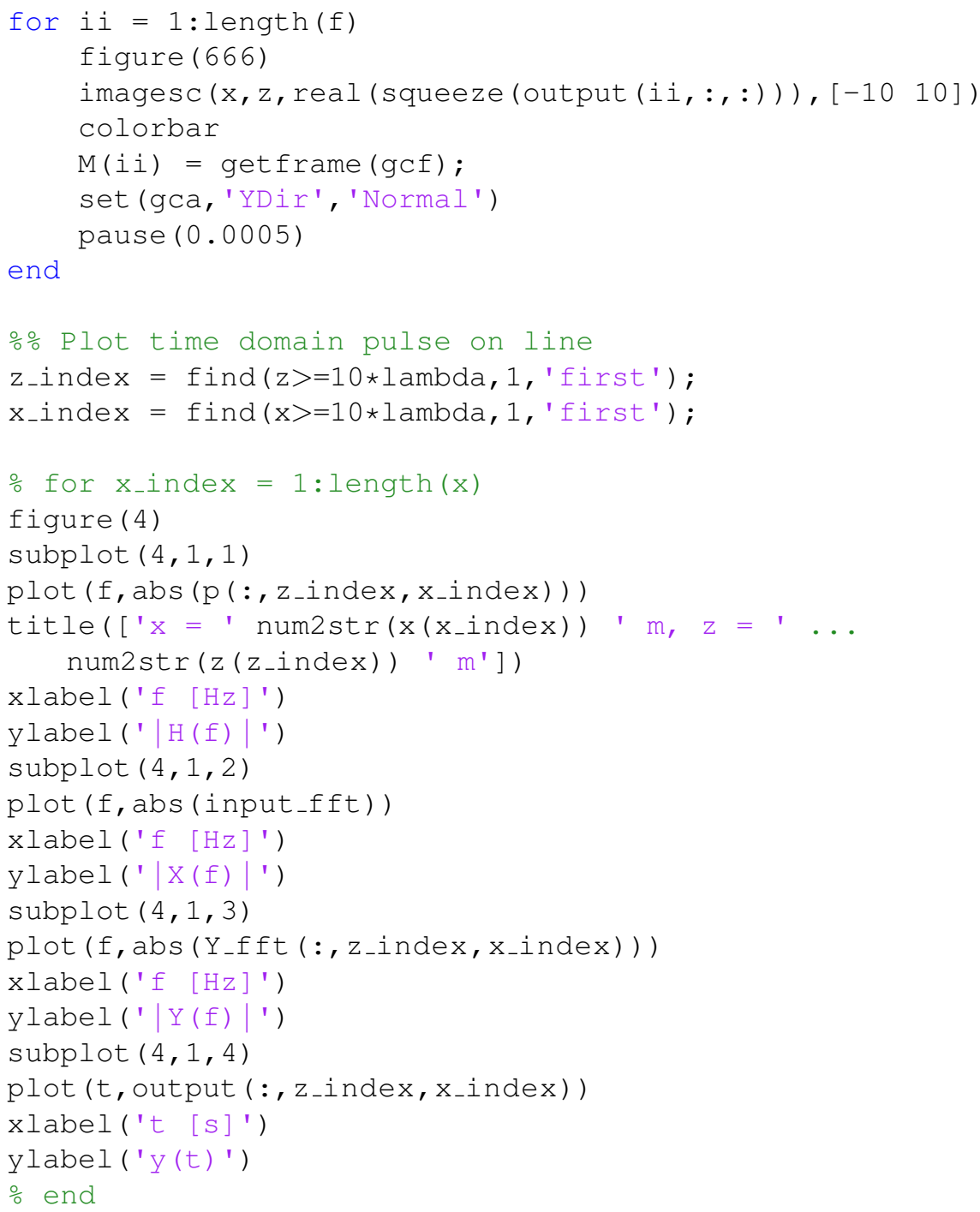




\section{Appendix C}

\section{MATLAB Code for Heat Transfer Model}

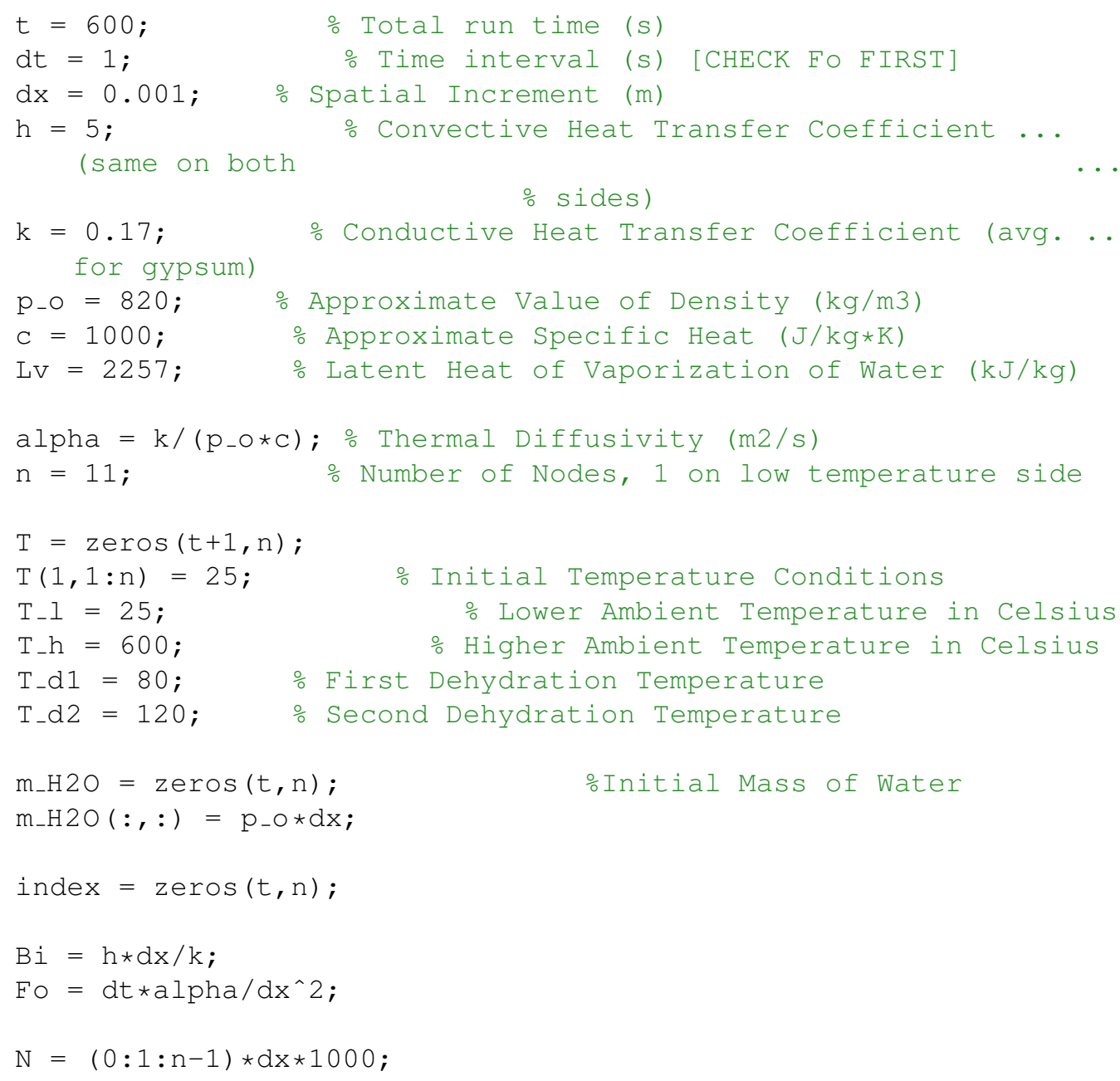




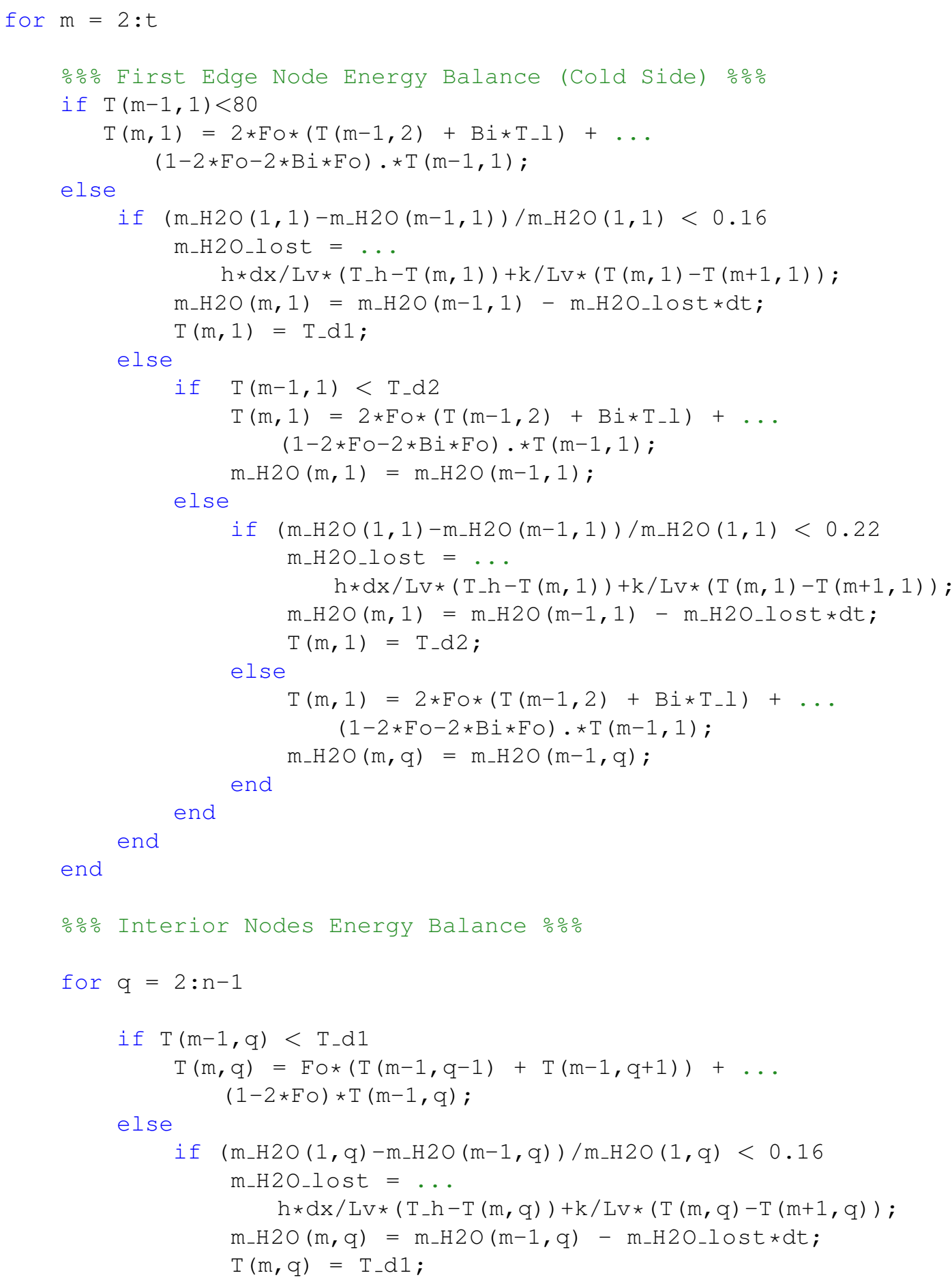




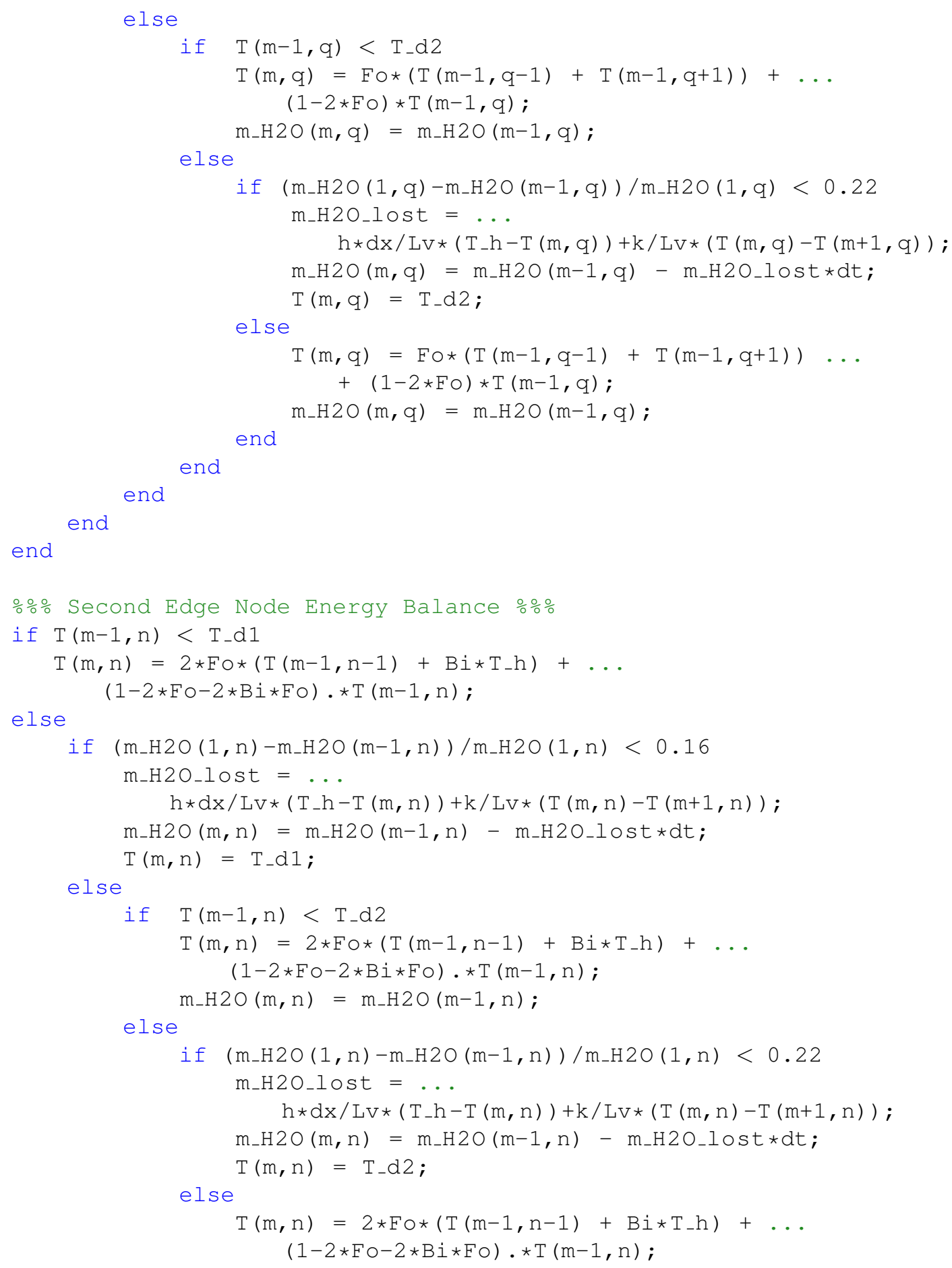




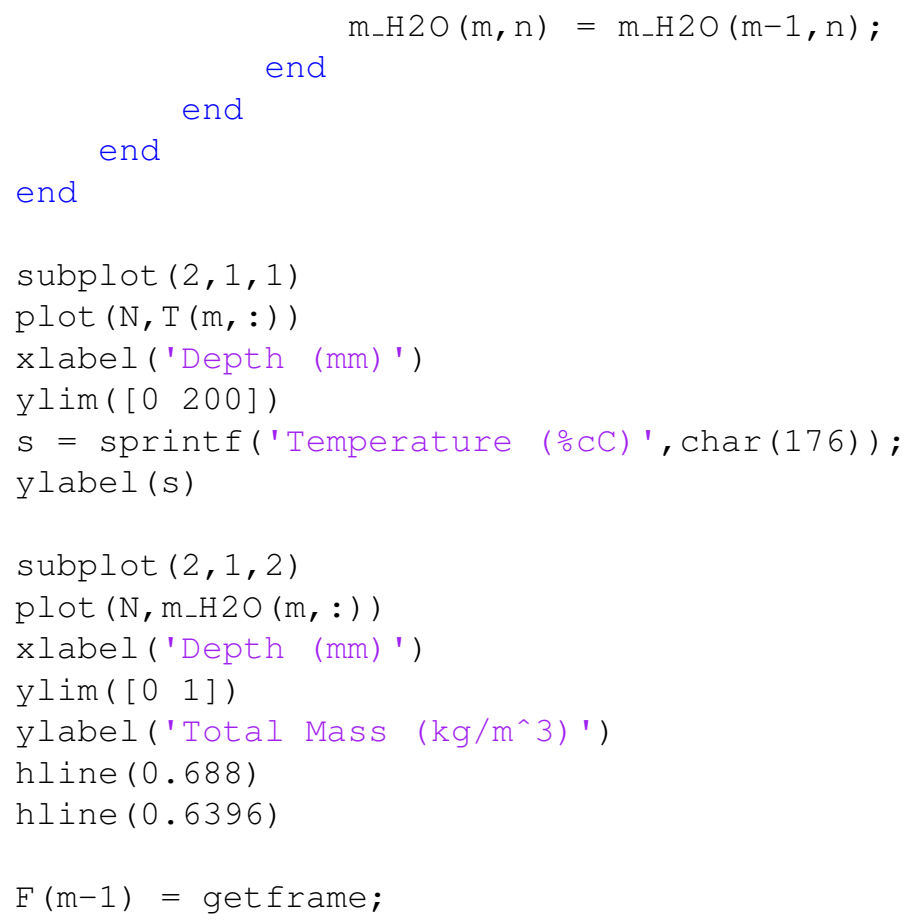




\section{Bibliography}

[1] K. Roche, "Evolving Safety Technology: Integrated PASS Devices," Fire Engineering, vol. 149, pp. 56-60, 1996.

[2] Fire Fighter Safety and Survival. Federal Emergency Management Agency, National Fire Academy, National Emergency Training Center, Emmitsburg, MD, 1986.

[3] L. Hall. Personal Letter, May 1979.

[4] L. Simross, "L.A. Firemen Protest Ban on Personal Alarm Devices," Los Angeles Times, vol. 4, p. 20, August 1979.

[5] C. Kiernan, "Personal alarm safety system." U.S. Patent 5,541,579, July 30, 1996.

[6] T. Pettit and T. Merinar, "Health Hazard Evaluation Report - Memphis Fire Department, Memphis, TN," tech. rep., Nat. Inst. of Occupational Health and Safety, Cincinnati, OH, June 1994.

[7] J. Routley, "Three firefighters die in Pittsburgh house fire," Tech. Rep. USFA-TR-078, U.S. Fire Admin, Emmitsburg, MD, Feb. 1995.

[8] L. Wise and P. Frank, "Self-contained breathing apparatus having a personal alert safety system integrated therewith." U.S. Patent 5,781,118, 
July 14, 1998.

[9] L. Stumberg and J. Fulton, "Integrated firefighter safety monitoring and alarm system." U.S. Patent 5,157,378, October 20, 1992.

[10] K. Ford, M. Habeeb, J. Suits, M. Abbasi, P. Wilson, and O. Ezekoye, "An analysis of firefighter personal safety alarm effectiveness on the fireground," Journal of the Acoustical Society of America, vol. 134, 2013.

[11] S. Wertman, "A Career Lieutenant and a Career Fire Fighter Found Unresponsive at a Residential Structure Fire - Connecticut," Tech. Rep. F2010-18, Nat. Inst. of Occupational Health and Safety, Cincinnati, OH, June 2011.

[12] M. Loflin, S. Miles, and T. Hales, "Career Fire Fighter Dies during FireFighting Operations at a Multi-family Residential Structure Fire - Massachusetts," Tech. Rep. F2011-31, Nat. Inst. of Occupational Health and Safety, Cincinnati, OH, Apr. 2013.

[13] S. Wertman, "Volunteer Lieutenant Killed and Two Fire Fighters Injured Following Bowstring Roof Collapse at Theatre Fire - Wisconsin," Tech. Rep. F2012-08, Nat. Inst. of Occupational Health and Safety, Cincinnati, OH, Oct 2012 .

[14] O. Ezekoye, P. Wilson, D. McFadden, J. Suits, M. Abbasi, C. Farmer, C. Grant, and M. Yin, "Evaluation and enhancement of fire fighter pass ef- 
fectiveness," tech. rep., The Fire Protection Research Foundation, Quincy, Massachusetts, December 2014.

[15] R. J. Urick, Principles of Underwater Sound. McGraw-Hill, Inc., 3rd ed., 1983.

[16] J. Suits, "Development of a Sonar Equation Formalism for Fireground Acoustics," Master's thesis, University of Texas at Austin, 2013.

[17] J. Suits, P. Wilson, and O. Ezekoye, "Background noise levels on the fireground (A)," Journal of the Acoustical Society of America, vol. 134, p. 4221, 2013.

[18] R. Tubbs, "Health Hazard Evaluation Report - Hamilton Fire Department, Hamilton, Ohio," Tech. Rep. HETA 89-0026-2495, Nat. Inst. of Occupational Health and Safety, Cincinnati, OH, 1995.

[19] U. Reischl, H. Bair, and P. Reischl, "Fire fighter noise exposure," American Industrial Hygiene Association, vol. 40, pp. 482-489, 1979.

[20] R. Tubbs, "Health Hazard Evaluation Report - Newburgh Fire Department, Newburgh, NY," Tech. Rep. HETA 81-059-1045, Nat. Inst. of Occupational Health and Safety, Cincinnati, OH, 1982.

[21] R. Tubbs, "Health Hazard Evaluation Report - City of New York Fire Department, New York, NY," Tech. Rep. HETA 81-459-1603, Nat. Inst. of Occupational Health and Safety, Cincinnati, OH, July 1985. 
[22] "American national standard specification for outdoor measurement of sound pressure level." ANSI Standard S12.18-2009.

[23] R. Neitzel, O. Hong, P. Quinlan, and R. Hulea, "Pilot task-based assessment of noise levels among firefighters," International Journal of Industrial Ergonomics, vol. 43, pp. 479-486, 2013.

[24] "American national standard specification for octave-band and fractionaloctave-band analog and digital filters." ANSI Standard S1.11-2004.

[25] "American national standard specification for sound level meters." ANSI Standard S1.14-1983.

[26] Kinsler, L.E. and Frey, A.R., Fundamentals of Acoustics. Hoboken, NJ: John Wiley \& Sons, Inc., 2nd ed., 1962.

[27] D. T. Blackstock, Fundamentals of Physical Acoustics. Hoboken, NJ: John Wiley \& Sons, Inc., 2000.

[28] M. Abbasi, O. Ezekoye, and C. Grant, "Can You Hear Me Now? The Next Generation of Fire Fighter PASS," Presented at the 2014 NFPA Conference \& Expo, Las Vegas, Nevada, 2014.

[29] A. Scharine, "The impact of helmet design on sound detection and localization (A)," Journal of the Acoustical Society of America, vol. 117, p. 2561, 2005. 
[30] A. Scharine, "Degradation of auditory localization performance due to helmet ear coverage: The effects of normal acoustic reverberation," Tech. Rep. ARL-TR-4879, Army Research Laboratory, Aberdeen Proving Ground, Maryland, July 2009.

[31] Q. Li, J. Haijeck, and T. Burchfield, "Law enforcement head-borne personal protective equipment hearing attenuation," Tech. Rep. ECBC-CR103, U.S. Army Research Office, Research Triangle Park, North Carolina, April 2009.

[32] A. Langbell, "Commercial Search Training Drill: Finding Lost Firefighters," Fire Engineering Mag. Web, October 28, 2014.

[33] J. Suits, C. Farmer, M. Abbasi, P. Wilson, and O. Ezekoye, "Personal alert safety system localization field tests with firefighters (A)," Journal of the Acoustical Society of America, vol. 136, p. 2166, 2014.

[34] C. Giguere and S. Abel, "Sound localization: Effects of reverberation time, speaker array, stimulus frequency, and stimulus rise/decay," Journal of the Acoustical Society of America, vol. 94, pp. 769-776, 1993.

[35] B. Rakerd and W. Hartmann, "Localization of noise in a reverberant environment," Auditory Signal Processing: Physiology, Psychophysics, and Models, pp. 348-354, 2005.

[36] R. Nagai, M. Fukamachi, and T. Nagatini, "Evacuation of crawlers and walkers from corridor through an exit," Physica A, vol. 367, pp. 449-460, 
2006.

[37] J. Davis, R. Tang, J. Sesek, and S. Gallagher, "Evaluating Firefighter Crawling Performance in a Controlled Environment," in Proceedings of the 5th International Conference on Applied Human Factors and Ergonomics, AHFE, July 2014.

[38] M. Long, Architectural Acoustics. Burlington, MA: Elsevier Academic Press, Inc., 2006.

[39] Autodesk, "Autodesk Ecotect Analysis: Visualize sustainable design." Brochure, 2011.

[40] H. Lehnert, "Systematic Errors of the Ray-Tracing Algorithm," Applied Acoustics, vol. 38, pp. 207-221, 1993.

[41] T. Funkhouser, "A beam tracing method for interactive architectural acoustics," The Journal of the Acoustical Society of America, vol. 115, pp. 739-756, 2004.

[42] T. Funkhouser, I. Carlbom, G. Elko, G. Pingali, M. Sondhi, and J. West, "A beam tracing approach to acoustic modeling for interactive virtual environments," in ACM Computer Graphics, SIGGRAPH'98 Proceedings, pp. 21-32, July 1998.

[43] G. Dhatt, G. Touzot, and E. Lefrancois, Finite Element Method. Hoboken, NJ: ISTE Ltd and John Wiley \& Sons, Inc., 2012. 
[44] M. Vorlander, Auralization: Fundamentals of Acoustics, Modelling, Simulation, Algorithms and Acoustic Virtual Reality. Springer-Verlag Berlin Heidelberg, 1st ed., 2008.

[45] R. Lyons, Understanding Digital Signal Processing. Prentice Hall, 2001.

[46] Q. Yu, "Thermal Properties and Microstructure of Gypsum Board and its Dehydration," Fire and Materials, vol. 36, pp. 575-579, 2012.

[47] C. Nguong, "Calcination of Gypsum Plasterboard under Fire Exposure," Master's thesis, University of Canterbury, 2004.

[48] F. P. Incropera and D. P. DeWitt, Fundamentals of Heat and Mass Transfer. New York, NY: Wiley, 6th ed., 2007.

[49] M. Lossi and E. Viveiros, "Sound Insulation of Gypsum Board in Practice," in The 2005 Congress and Exposition on Noise Control Engineering.

[50] CertainTeed, "Noise Control for Buildings: Guidelines for Acoustical Problem Solving." Brochure, 2010. 